\title{
A STEP BEYOND KEMPERMAN'S STRUCTURE THEOREM
}

\author{
DAVID J. GRYNKIEWICZ
}

Abstract. We extend Kemperman's Structure Theorem by completely characterizing those finite subsets $A$ and $B$ of an arbitrary abelian group with $|A+B|=|A|+|B|$.

\section{INTRODUCTION}

Let $G$ be an abelian group, and let $A$ and $B$ be nonempty subsets of $G$. Their sumset is the set of all pairwise sums, i.e., $A+B=\{a+b \mid a \in A, b \in B\}$. For a set $\mathcal{S}$ of subsets of $G$, define

$$
d^{\subseteq}(A, \mathcal{S})=\min _{B \in \mathcal{S}}\left\{d^{\subseteq}(A, B)\right\},
$$

where $d \subseteq(A, B)=|B \backslash A|$, if $A \subseteq B$, and $d \subseteq(A, B)=\infty$ otherwise. Hence $d \subseteq(A, \mathcal{S})$ measures how far away as a subset the set $A$ is from the sets $B \in \mathcal{S}$.

It is the central problem of inverse additive number theory to describe the structure of those pairs of subsets $A$ and $B$ with $|A+B|$ small. Such descriptions often prove useful to other related areas of mathematics (see [34]). One of the classical breakthroughs in the area was the complete recursive description given by Kemperman [28] of the 'critical pairs' in an abelian group, i.e., those finite, nonempty subsets $A$ and $B$ such that $|A+B|<|A|+|B|$. Among other applications - including results in graph theory [22] and zero-sum additive theory [9] - Kemperman's Structure Theorem (KST), whose statement we delay until later, yields the descriptions of those subsets of a locally compact abelian group whose Haar measure of the sumset fails to satisfy the triangle inequality [28] [29]. Other applications may also be found in [37] [33] [12] [13].

Unfortunately, a perception of complexity has limited more widespread applications of KST. In fact, several papers have been published with such goals as the simplification of KST [32], the creation of alternative methods for dealing with critical pairs [20] [21], and the clarification of the use of KST in practice [10] [33].

For precise structure of $A$ and $B$ when $A+B$ is small, there has been almost no progress in the subsequent $45+$ years following KST for an arbitrary abelian group. However, with weaker results and particular groups, some related successes were seen.

In the case $G=\mathbb{Z}$ with $|A| \geq|B|$, then (currently, a few technical restrictions are also needed)

$$
|A+B|=|A|+|B|+r \leq|A|+2|B|-4+\epsilon
$$

implies that $d \subseteq\left(A, \mathcal{A P}_{d}\right), d \subseteq\left(B, \mathcal{A P}_{d}\right) \leq r+1$ for some $d$, where $\mathcal{A P}_{d}$ is the set of arithmetic progressions with difference $d$, and $\epsilon$ equals 0 or 1 depending on a structural condition between $A$ and $B$ [34]. Thus $A$ and $B$ must be large subsets of arithmetic progressions with the same difference.

In the case $G=\mathbb{Z} / p \mathbb{Z}$, where $p$ is prime, then the critical pairs-with $|A| \geq|B|>1$ and $|A+B| \leq p-2$ (to avoid three very special degenerate examples) - consist of arithmetic progressions with the same difference [39] [40]. Under some additional restrictions on the cardinality of $A$, and assuming $A=B$, then a result of Freiman shows that $|A+A| \leq 2|A|+r \leq 2.4|A|$ implies $d \subseteq\left(A, \mathcal{A P} \mathcal{P}_{d}\right) \leq r+1$ for some $d$ [4] [5] [34]; in

This Research was supported in part by the National Science Foundation, as a Math and Physical Sciences Distinguished International Postdoctoral Research Fellow, under grant DMS-0502193, and also in part by the Austrian Science Fund FWF, Project Number M1014-N13. 
other words, the above result from $\mathbb{Z}$ holds for $A=B$, by imposing some moderate conditions, in $\mathbb{Z} / p \mathbb{Z}$ as well. The same result is also known for $\mathbb{Z} / p \mathbb{Z}$ under the more general assumption of (1), provided extremely severe conditions are imposed on the cardinalities of $A$ and $B$ [1]. The extent to which the result holds in $\mathbb{Z} / p \mathbb{Z}$ without unnecessary assumptions on the cardinalities is still quite open. Little is known beyond the case $|A+B|=|A|+|B|$, for which Hamidoune and Rødseth established $d \subseteq\left(A, \mathcal{A} \mathcal{P}_{d}\right), d \subseteq(B, \mathcal{A P} d) \leq 1$, with only the assumption $|A+B| \leq p-4$ and the removal of $\epsilon$ from (1) (recall $|A| \geq|B|$ ) [19]; and the case $|A+B|=|A|+|B|+1$, for which $d^{\subseteq}\left(A, \mathcal{A P}_{d}\right), d^{\subseteq}\left(B, \mathcal{A P} \mathcal{P}_{d}\right) \leq 2$ was shown by Hamidoune, Serra, and Zemor under similar assumptions with $p>51$ [17]. Concerning slightly more general abelian groups, for $A \subseteq \mathbb{Z} / m Z$ with $|A+A|<2.04|A|$, Deshouillers and Freiman obtained a rough description of $A$ involving computed but very large constants [3].

In this paper, we move one step beyond KST by completing the description of all subsets $A$ and $B$ that exactly achieve (rather than fail to achieve) the triangle inequality, namely for which $|A+B|=|A|+|B|$.

Our main result is Theorem 4.1 (whose statement we also delay until further notation and concepts have been developed), which shows that with a few noted exceptions - all but one in the same vein as the original recursive description of $\mathrm{KST}$ - then such $A$ and $B$ must be large subsets of a critical pair; more specifically, there must exist $A^{\prime} \supseteq A$ and $B^{\prime} \supseteq B$ such that $\left|A^{\prime}+B^{\prime}\right|=\left|A^{\prime}\right|+\left|B^{\prime}\right|-1$, and which contain $A$ and $B$ each with at most one hole, i.e., $\left|A^{\prime} \backslash A\right| \leq 1$ and $\left|B^{\prime} \backslash B\right| \leq 1$. Thus in the case $G=\mathbb{Z} / p \mathbb{Z}$, with $p$ prime, Theorem 4.1 generalizes the prime case completed by Hamidoune and Rødseth, and is the corresponding composite extension of KST. Theorem 4.1 and KST will also yield necessary and sufficient conditions for $|A+B|=|A|+|B|$.

We remark that Hamidoune, Serra and Zemor recently established a particular case of Theorem 4.1, under a series of added assumptions, including that $\operatorname{gcd}(|G|, 6)=1$, that $A$ be a generating subset, that the order of every element of $A \backslash 0$ be at least $|A|+1$, and that a few smaller technical assumptions also hold [16] - the hypotheses needed for their result, particularly the assumption on the order of elements, parallel other first-attempt generalizations of additive results from the prime order case to the more general abelian group setting (see [2] [26] [27] for other such examples).

Additionally, the results and methods developed for this paper have since been adapted to prove a version of Pollard's Theorem for t-representable sums valid in an arbitrary abelian group (the original result was only for cyclic groups of prime order) [35] [34] [15], have inspired Y. O. Hamidoune to further develop the iso-perimetric method to the point where it can prove KST [23] [24], are now being used to extend the results for $\mathbb{Z}$ discussed below (1) [14], and have been used to prove a conjecture of Jungić, Nešetřil and Radoičić [25] concerning rainbow free colorings of abelian groups [18]. The careful reader will note that the proof of Theorem 4.1, with a few minor modifications and many simplifications, can also be used to prove KST and Kneser's Theorem.

\section{Preliminaries}

We will make heavy use of the interpretation of KST given in [10] (or in [11], where the explanations are slightly extended, including the expansion of a minor omission in comment (c.12) of [10]), some of which may also be found in [33]. First we begin by describing many of the important definitions and notation that we will use.

Let $G$ be an abelian group. A subset $A \subseteq G$ is $H$-periodic if $A$ is a union of $H$-cosets, with $H$ a subgroup (referred to as the period). Note that every set is $H$-periodic with $H$ the trivial group. If $A$ is $H$-periodic with $H$ a nontrivial subgroup, then $A$ is periodic, and otherwise $A$ is aperiodic. Note that $A$ being $H$-periodic is equivalent to $A+H=A$. Hence if $A$ is $H$-periodic, then so is $A+B$. The maximal subgroup for which 
$A$ is periodic is the stabilizer of $A$, denoted $H(A)=\{g \in G \mid g+A=A\}$. An $H$-hole in $A$ is an element of $(A+H) \backslash A$, and when clear, $H$ will be dropped from the notation. A punctured periodic set is a set $A$ such that $A \cup\{\gamma\}$ is periodic for some $\gamma \notin A$, i.e., $A$ contains exactly one $H$-hole for some nontrivial $H$. We remark that a punctured periodic set cannot be periodic (as for instance shown in [10] [11]). We use $\phi_{H}: G \rightarrow G / H$ to denote the natural homomorphism. Note that if $H=H(A)$, then $\phi_{H}(A)$ is aperiodic. One of the foundational results of additive theory is the following result of Kneser [30] [31] [28] [34].

Kneser's Theorem. Let $G$ be an abelian group, and let $A_{1}, A_{2}, \ldots, A_{n}$ be a collection of finite, nonempty subsets of $G$. If $H=H\left(\sum_{i=1}^{n} A_{i}\right)$, then $\left|\sum_{i=1}^{n} \phi_{H}\left(A_{i}\right)\right| \geq \sum_{i=1}^{n}\left|\phi_{H}\left(A_{i}\right)\right|-n+1$.

Note that if $H=H(A+B)$ and $\rho=|A+H|-|A|+|B+H|-|B|=d^{\subseteq}(A, A+H)+d^{\subseteq}(B, B+H)$ is the number of holes in $A$ and $B$, then Kneser's Theorem implies (by multiplying all terms by $|H|$ ) that $|A+B| \geq|A|+|B|-|H|+\rho$. Consequently, if either $A$ or $B$ contains a unique element from some $H$-coset, then $|A+B| \geq|A|+|B|-1$. Also, if $|A+B| \leq|A|+|B|-1$, then equality holds in the bound from Kneser's Theorem (else $\left.|A+B| \geq|H|\left(\left|\phi_{H}(A)\right|+\left|\phi_{H}(B)\right|\right) \geq|A|+|B|\right)$.

Given $a_{i} \in A$ and a subgroup $H$, we use $A_{a_{i}, H}$ to denote $\left(a_{i}+H\right) \cap A$, with the $H$ dropped from the notation when clear. If $A_{a_{i}} \neq a_{i}+H$, then $A_{a_{i}}$ is a partially filled $H$-coset. An $H$-decomposition of $A$ is a partition $A_{a_{1}} \cup \ldots \cup A_{a_{l}}$ of $A$ with $a_{i} \in A$. The complement of $A$ is denoted $\bar{A}:=G \backslash A$, and we use $\langle A\rangle$ to denote the subgroup generated by $A$, which, when $0 \in A$, is the smallest subgroup $H$ such that $\left|\phi_{H}(A)\right|=1$.

A quasi-periodic decomposition of $A$ with quasi-period $H$, where $H$ is a nontrivial subgroup, is a partition $A_{1} \cup A_{0}$ of $A$ into two disjoint (each possibly empty) subsets such that $A_{1}$ is $H$-periodic or empty, and $A_{0}$ is a subset of an $H$-coset. Note every set has a quasi-periodic decomposition with $H=G$ and $A_{1}=\emptyset$. A set $A \subseteq G$ is quasi-periodic if $A$ has a quasi-periodic decomposition $A=A_{1} \cup A_{0}$ with $A_{1}$ nonempty. Given a quasi-periodic decomposition $A_{1} \cup A_{0}$ with quasi-period $H$, then $A_{1}$ is the periodic part of the decomposition, and $A_{0}$ is the aperiodic part (although it may be periodic if $A$ is periodic). Such a decomposition is reduced if $A_{0}$ is not quasi-periodic. Note that if $A$ is finite and has a quasi-periodic decomposition $A_{1} \cup A_{0}$ with quasi-period $H$, then $A$ has a reduced quasi-periodic decomposition $A_{1}^{\prime} \cup A_{0}^{\prime}$ with quasi-period $H^{\prime} \leq H$ and $A_{0}^{\prime} \subseteq A_{0}$ (see also [10] [11]).

An arithmetic progression with difference $d \in G \backslash 0$ and length $l \in \mathbb{N}$ is a set of the form $\left\{a_{0}, a_{0}+\right.$ $\left.d, \ldots, a_{0}+(l-1) d\right\}$, where $a_{0} \in G$. The terms $a_{0}$ and $a_{0}+(l-1) d$ are the end terms (relative to $d$ ) in the progression, with $a_{0}$ the first term and $a_{0}+(l-1) d$ the last term (note these terms are uniquely defined, relative to $d$, for $l<\langle d\rangle)$. An arithmetic progression with difference $d$ is also an arithmetic difference with difference $-d$, with the first and last terms interchanged. A $d$-component of a set $A$ is a maximal arithmetic progression with difference $d$ contained in $A$. We use $c_{d}(A)$ to denote the number of aperiodic $d$-components of $A$. Note that if a $d$-component is periodic, then it must be a $\langle d\rangle$-coset. Hence $c_{d}(A)=|A+\{0, d\}|-|A|$. If $c_{d}(A)=1$, then $A$ is a quasi-progression with difference $d$.

Assuming $0 \in A \cap B$, for $i \geq 0$ we define the set $N_{i}(A, B)$ by

- $N_{0}(A, B)=A$,

- $N_{i}(A, B)=(A+i B) \backslash(A+(i-1) B)$ for $i \geq 1$,

where $0 B=\{0\}$ and $i B=\underbrace{B+\ldots+B}_{i}$ for $i \geq 1$. If the sets $A$ and $B$ are clear, they will often be dropped from the notation. For $x \in G$, let

$$
r_{A, B}(x)=|(x-A) \cap B| .
$$

Hence $r_{A, B}(x)=r_{B, A}(x)$ is the number of representations of $x=a+b$ with $a \in A$ and $b \in B$, and so $x$ is a unique expression element in $A+B$ when $r_{A, B}(x)=1$. For $U \subseteq B, i \geq 1$, and $N_{i}$ nonempty, we use 
$N_{i}^{U}$ to denote the set of all elements $x \in A+i B$ such that $(x-(A+(i-1) B) \cap B=U$, and we define $N_{i}^{\leq U}=\bigcup_{V \subseteq U} N_{i}^{V}$. Hence $A+(i-1) B+(B \backslash U)=(A+i B) \backslash N_{i}^{\leq U}$. In particular, $\left|N_{1}^{b}(A, B)\right|$ is the number of $a \in A$ with $a+b$ a unique expression element in $A+B$. The sets $N_{i}$ were first introduced in [36] in connection with small sumsets in $\mathbb{Z} / p \mathbb{Z}$, with $p$ prime, and have since shown themselves to be quite useful [16]. We remark that our definitions of $N_{i}^{U}$ and $N_{i}^{\leq U}$ differ slightly from those in [36], though they coincide when $0 \notin U$.

Let $\mathcal{P}$ be the set of periodic subsets of $G$, let $\mathcal{Q P}$ be the set of quasi-periodic subsets of $G$, let $\mathcal{Q P} \mathcal{P}_{H}$ be the set of quasi-periodic subsets of $G$ with quasi-period $H$, let $\mathcal{P}_{H}$ be the set of $H$-periodic subsets of $G$, let $\mathcal{A P}$ be the set of arithmetic progressions, let $\mathcal{A} \mathcal{P}_{d}$ be the set of arithmetic progressions with difference $d$, and let $\mathcal{Q} \mathcal{A} \mathcal{P}_{d}$ be the set of quasi-progressions with difference $d$. Note that $\mathcal{P} \subseteq \mathcal{Q P}$.

We will need the following basic proposition [34] [28]. Note that if $G$ is finite and $|A|+|B| \geq|G|+r$, then Proposition 2.1 implies $r_{A, B}(g) \geq r$ for every $g \in G$.

Proposition 2.1. Let $A$ and $B$ be nonempty, finite subsets of an abelian group $G$, and let $r \in \mathbb{Z}$.

(i) If $G$ is finite, and $|A|+|B| \geq|G|+1$, then $A+B=G$.

(ii) If $|A+B|<|A|+|B|-r$, then $r_{A, B}(g)>r$ for every $g \in A+B$.

We next define four types (I)-(IV) of elementary pairs which form the basic building blocks for all critical pairs. We say the pair of nonempty, finite subsets $(A, B)$ of the abelian group $G$ is of type:

(I) if $|A|=1$ or $|B|=1$;

(II) if $|A| \geq 2,|B| \geq 2$, and $A$ and $B$ are arithmetic progressions with common difference $d$, where the order of $d$ is at least $|A|+|B|-1$;

(III) if $A \subseteq a+H$ and $B \subseteq b+H$ (for some $a \in A, b \in B$ and $H \leq G),|A|+|B|=|H|+1$ (and thus $A+B=a+b+H$ by proposition 2.1), and $a+b$ is the only unique expression element in $A+B$;

(IV) if $A \subseteq a+H$ and $B \subseteq b+H$ (for some $a \in A, b \in B$ and $H \leq G$ ), $A+B$ contains no unique expression elements, $A$ and $B$ are aperiodic, and $A=g-(b+H) \backslash B$ (for some $g \in G$ ).

Note, in all four cases, that $|A+B|=|A|+|B|-1$ for an elementary pair $(A, B)$; in particular, we have $|A+B|=|H|-1$ when $(A, B)$ has type (IV); also, the additional conditions on unique expression elements for type (III) and (IV) pairs imply $|A|,|B| \geq 3$ (see [10] [28]). We now give the statement of KST [28].

Kemperman Structure Theorem (KST). Let $G$ be a finite abelian group, and let $A, B \subseteq G$ be finite and nonempty. Now $|A+B|=|A|+|B|-1$ and either $A+B$ is aperiodic or contains a unique expression element if and only if there exist quasi-periodic decompositions $A=A_{1} \cup A_{0}$ and $B=B_{1} \cup B_{0}$ with common quasi-period $H$ and $A_{0}$ and $B_{0}$ nonempty, such that:

(i) $\phi_{H}\left(A_{0}\right)+\phi_{H}\left(B_{0}\right)$ is a unique expression element in $\phi_{H}(A)+\phi_{H}(B)$;

(ii) $\left|\phi_{H}(A+B)\right|=\left|\phi_{H}(A)\right|+\left|\phi_{H}(B)\right|-1$;

(iii) if $a+b \in A+B$ is a unique expression element with $a \in A$ and $b \in B$, then $a \in A_{0}$ and $b \in B_{0}$;

(iv) $\left(A_{0}, B_{0}\right)$ is an elementary pair of type (I), (II), (III) or (IV).

Condition (iii) was not stated in Kemperman's original paper, but can be derived from KST as shown in [10] [11]. Conditions (i) and (ii) imply that the pair $\left(\phi_{H}(A), \phi_{H}(B)\right)$ satisfies the hypothesis of KST. Hence repeated application of KST modulo the quasi-period yields a complete recursive description of the pair $(A, B)$. This gives rise to a chain of subgroups

$$
0=H_{0}<H=H_{1}<H_{2}<\ldots<H_{n}=G,
$$

where $H_{i} / H_{i-1}$ is the quasi-period given by KST after $i$ iterations. Conditions (i) and (iii) ensure 'proper alignment' during this recursive process, namely that $\phi_{H}\left(A_{0}\right)$ and $\phi_{H}\left(B_{0}\right)$ will always be elements contained 
in the aperiodic part of the quasi-periodic decomposition arising from the subsequent iterative application of KST to $\left(\phi_{H}(A), \phi_{H}(B)\right)$; otherwise put, $\phi_{H_{j-1}}\left(A \cap\left(A_{0}+H_{j}\right)\right)$ is the aperiodic part of the $j$-the iterated Kemperman decomposition, and likewise for $B$. Consequently, KST induces partitions (allowing empty parts) $A=A_{n} \cup A_{n-1} \cup \ldots \cup A_{1} \cup A_{0}$ and $B=B_{n} \cup B_{n-1} \cup \ldots \cup B_{1} \cup B_{0}$, such that $A_{n}=B_{n}=\emptyset, A_{0}$ and $B_{0}$ are nonempty, and

$$
\phi_{H_{j-1}}(A)=\phi_{H_{j-1}}\left(A_{n} \cup \ldots \cup A_{j}\right) \bigcup \phi_{H_{j-1}}\left(A_{j-1} \cup \ldots \cup A_{0}\right),
$$

and

$$
\phi_{H_{j-1}}(B)=\phi_{H_{j-1}}\left(B_{n} \cup \ldots \cup B_{j}\right) \bigcup \phi_{H_{j-1}}\left(B_{j-1} \cup \ldots \cup B_{0}\right)
$$

are the quasi-periodic decompositions with quasi-period $H_{H_{j}} / H_{H_{j-1}}$ given by KST after $j$ applications, while

$$
A=\left(A_{n} \cup \ldots \cup A_{j}\right) \bigcup\left(A_{j-1} \cup \ldots \cup A_{0}\right),
$$

and

$$
B=\left(B_{n} \cup \ldots \cup B_{j}\right) \bigcup\left(B_{j-1} \cup \ldots \cup B_{0}\right)
$$

are also quasi-period decompositions of $A$ and $B$ with common quasi-period $H_{j}$. From this one readily derives the dual formulation of KST given in [33].

At first it might appear that the critical pairs with $A+B$ periodic and having no unique expression element, including the cases when $|A+B|<|A|+|B|$, are not fully covered by KST. However these cases are easily reduced to those covered by KST. This is because, letting $H=H(A+B)$, it follows in view of Kneser's Theorem that $\left|\phi_{H}(A+B)\right|=\left|\phi_{H}(A)\right|+\left|\phi_{H}(B)\right|-1$, with $\phi_{H}(A+B)$ aperiodic and $A+B$ containing exactly $|A+B|+|H|-|A|-|B|$ holes. Thus KST is used to describe the pair $\left(\phi_{H}(A), \phi_{H}(B)\right)$, and then $A$ and $B$ are obtained from $A+H$ and $B+H$ by deleting $|A+B|+|H|-|A|-|B| \leq|H|-1$ elements. In view of Proposition 2.1, these deleted elements can be placed anywhere in the sets $H+A$ and $H+B$ with the resulting sets being critical. Thus there is nothing to say about the location of these holes.

It is natural to wonder if a pair $(A, B)$ could have more than one quasi-periodic decomposition that satisfies KST, in other words, how unique is the representation given by KST. This question is addressed in [10] [11]. We provide a short summary. The type of a critical pair $(A, B)$ is unique and not dependent on the choice of quasi-periodic decompositions that satisfy $\mathrm{KST}$. If the pair $(A, B)$ has type (II), then there is a unique pair of quasi-periodic decompositions that satisfies KST. The same is true (in view of the added condition (iii)) for type (I). In both cases, the quasi-period $H$ may not be unique, but can always be taken to be maximal, subject to the conditions of KST. There is slightly more freedom for types (III) and (IV), but in both cases there is a natural canonical choice. For type (III), the quasi-period may always be taken to be $H(A+B)$. This ensures that type (III) will not occur twice in a row when recursively iterating KST. Type (IV) can only occur in the first iteration of KST (since a type (IV) pair has no unique expression element), and implies that $A+B$ is a punctured $H$-periodic set with $|H| \geq 6$. These conditions imply that there is a unique $\gamma \in \overline{A+B}$ such that $(A+B) \cup\{\gamma\}$ is periodic, and $H$ may always be taken to be the maximal period of $(A+B) \cup\{\gamma\}$. This ensures that type (III) not follow type (IV) when iterating KST. Hence, for all types, the quasi-period $H$ given by KST may always be taken to be maximal, and the resulting quasi-period decompositions that satisfy KST will be referred to as the Kemperman decompositions.

Regarding the sequence of possible types, there is some restriction on when type (I) can occur twice in a row, assuming $H$ chosen maximally. Namely, if $A=A_{1} \cup A_{0}$ and $B=B_{1} \cup B_{0}$ are the Kemperman decompositions of $A$ and $B$ with common quasi-period $H$, and if $\phi_{H}(A)=\phi_{H}\left(A_{1}^{\prime}\right) \cup \phi_{H}\left(A_{0}^{\prime}\right)$ and $\phi_{H}(B)=$ $\phi_{H}\left(B_{1}^{\prime}\right) \cup \phi_{H}\left(B_{0}^{\prime}\right)$ are the Kemperman decompositions of $\phi_{H}(A)$ and $\phi_{H}(B)$, then we cannot have both $(A, B)$ of type (I) with $\left|A_{0}\right|=1$, and also $\left(\phi_{H}(A), \phi_{H}(B)\right)$ of type (I) with $\left|\phi_{H}\left(A_{0}^{\prime}\right)\right|=1$ - this is the main 
step in the proof of Proposition 2.2 in [10], and likewise for Proposition 5.3 in [11]. Finally, it should also be observed that if $A$ is not quasi-periodic and $\langle-\alpha+A\rangle=G$, with $\alpha \in A$, then $H=G$.

We will also need the following two simple propositions that, like the proofs given here, are minor variations on two results from [36].

Proposition 2.2. Let $G$ be an abelian group, let $X, Y \subseteq G$ be finite and nonempty with $0 \in X \cap Y$, and let $i \geq 1$. If $r_{X, Y}(z) \geq t$ for every $z \in X+Y$, then $r_{X+(i-1) Y, Y}(z) \geq t$ for every $z \in X+i Y$.

Proof. It suffices to prove the case $i=2$, as the other cases follow by repeated application. Let $y, y^{\prime} \in Y$ and $x \in X$. Since $r_{X, Y}(z) \geq t$ for $z \in X+Y$, let $\left\{x_{i}+y_{i}\right\}_{i=1}^{t}$ be distinct representations of $x+y$, with $x_{i} \in X$ and $y_{i} \in Y$. Hence $\left\{\left(x_{i}+y^{\prime}\right)+y_{i}\right\}_{i=1}^{t}$ are distinct representations of $x+y+y^{\prime} \in X+2 Y$ with $x_{i}+y^{\prime} \in X+Y$ and $y_{i} \in Y$, whence $r_{X+Y, Y}(z) \geq t$ for every $z \in X+2 Y$.

Proposition 2.3. Let $G$ be an abelian group, let $X, Y \subseteq G$ be finite and nonempty with $0 \in X \cap Y$, and let $i \geq 1$. If $U \subseteq Y$, then $N_{i+1}^{U}(X, Y)-U \subseteq N_{i}^{\leq U}(X, Y)$.

Proof. Let $x \in N_{i+1}^{U}$. Hence by definition $U$ is the maximal subset of $Y$ such that $x-U \subseteq X+i Y$. If $x-U \nsubseteq N_{i}^{\leq U}$, then for some $u \in U$, it follows (see the comment after the definition of $N_{i}^{\leq U}$ ) that $x-u=z \in X+i Y$, with $z=u^{\prime}+x^{\prime}$ for some $u^{\prime} \in Y \backslash U$ and $x^{\prime} \in X+(i-1) Y$. Thus $x-u^{\prime}=u+x^{\prime} \in X+i Y$. Hence, since $u^{\prime} \notin U$, this contradicts the maximality of $U$.

We conclude the section with one last important concept. Given a pair of subsets $A$ and $B$ of an abelian group $G$, we say that $A$ is non-extendible, with respect to $B$, if

$$
\left(A \cup\left\{a_{0}\right\}\right)+B \neq A+B \text {, for all } a_{0} \in \bar{A} .
$$

We say $A$ is extendible otherwise. We will call the pair $(A, B)$ non-extendible if both $A$ and $B$ are nonextendible (with respect to each other), and extendible otherwise. Note that in general

$$
-B+\overline{A+B} \subseteq \bar{A}
$$

since otherwise $-b+c_{0}=a$ for some $b \in B, c_{0} \in \overline{A+B}$, and $a \in A$, implying $c_{0}=a+b \in A+B$, contradicting $c_{0} \in \overline{A+B}$. However, $A$ being non-extendible is in fact equivalent to equality holding.

Proposition 2.4. For subsets $A$ and $B$ of an abelian group, $A$ is non-extendible, with respect to $B$, if and only if

$$
-B+\overline{A+B}=\bar{A}
$$

In particular, the pair $(A, B)$ is non-extendible if and only if both (4) and

$$
-A+\overline{A+B}=\bar{B}
$$

hold, and both the pairs $(-A, \overline{A+B})$ and $(-B, \overline{A+B})$ are also non-extendible.

Proof. Suppose $A$ in non-extendible. Let $a_{0} \in \bar{A}$. In view of (2), it follows that there exists $b \in B$ and $c_{0} \in \overline{A+B}$ such that $a_{0}+b=c_{0}$, whence $a_{0}=-b+c_{0} \in-B+\overline{A+B}$. Thus $\bar{A} \subseteq-B+\overline{A+B}$, and equality follows in view of $(3)$.

On the other hand, if we suppose (4), then each $a_{0} \in \bar{A}$ can be written as $a_{0}=-b+c_{0}$, with $b \in B$ and $c_{0} \in \overline{A+B}$, implying $a_{0}+b=c_{0}$ is an element of $\left(A \cup\left\{a_{0}\right\}\right)+B$ not contained in $A+B$, whence (2) follows.

If $(A, B)$ is non-extendible, and $(-A, \overline{A+B})$ is extendible, then in view of (4), (5), and the first part of the proposition, it would follows that either $A+B \neq A+B$ or $-\overline{A+B}+B \neq-\bar{A}$. The former is clearly 
a contradiction, while in view of (4), the later contradicts the non-extendibility of $A$. The same argument applied to the pair $(-B, \overline{A+B})$ shows it to likewise be non-extendible, completing the proof.

When $G$ is finite, it is important to note that if $|A+B|=|A|+|B|+r$ with $B$ non-extendible, then proposition 2.4 implies that $|-A+\overline{A+B}|=|-A|+|\overline{A+B}|+r$ as well. Thus a non-extendible pair $(A, B)$ with $|A+B|=|A|+|B|+r$ is part of a triple of non-extendible pairs, all having their sumsets with cardinality exactly $r$ more than the bound given by the triangle inequality. This motivates the following definition. We say that the pair $A^{\prime}, B^{\prime} \subseteq G$ is a dual pair to $A, B \subseteq G$, with respect to $H \leq G$, if $A \subseteq a+H$ and $B \subseteq b+H$ (for some $a \in A$ and $b \in B$ ), $(A, B)$ is non-extendible, and either $A^{\prime}=g-A$ and $B^{\prime}=g^{\prime}+(a+b+H) \backslash(A+B)$ (for some $g, g^{\prime} \in G$ ), or $B^{\prime}=g-B$ and $A^{\prime}=g^{\prime}+(a+b+H) \backslash\left(A+B\right.$ ) (for some $g, g^{\prime} \in G$ ); in other words, up to translation, $A^{\prime}$ and $B^{\prime}$ are obtained by applying Proposition 2.4 to $A$ and $B$ when considered as subsets of $H$. In this language, a type (IV) pair is just the dual of a type (I) pair with respect to some finite $H \leq G$. The ideas behind Proposition 2.4 trace their roots back to Vosper [39], and can also be found in the isoperimetric method (since $k$-fragments are non-extendible) [20] [21].

\section{3. $A+B$ Versus $A-B$}

For the proof of our main result, we will need the following basic theorem, which can be viewed as generalization of the bound for Sidon sets and which greatly increases the potency of the Dyson $e$-transform in practice. Indeed, if $A=B,|T|=1$ and $k=1$ (the conditions for $A$ to be a Sidon set, given from the difference set point of view), then the familiar bound $|A+A| \geq \frac{|A|(|A|+1)}{2}$ follows from Theorem 3.1(iii) by noting that $x=|A|$.

Theorem 3.1 will allow us to conclude $|A+B|$ is large provided $r_{A,-B}(x)$ is small for most $x \in A-B$. This will be important as the proof of our main result uses (a variation of) the Dyson $e$-transform. Namely for $e \in A-B$, where $|A| \geq|B|$, we define $B(e)=(e+B) \cap A$ and $A(e)=(e+B) \cup A$. Then $|A(e)|+|B(e)|=$ $|A|+|B|$ while $A(e)+B(e) \subseteq e+A+B$. Our main strategy will be to apply induction to the pair $(A(e), B(e))$. However, we will encounter problems with this strategy if $|B(e)|$ is small for all $e$ such that $e+B \nsubseteq A$. In these cases, we will use Theorem 3.1 to show that $|A+B|$ is large instead. The bounds below have been crafted to take into account improvements gained from certain divisibility conditions, which increases their accuracy albeit at the expense of making the expressions appear much more complex. However, for applications of Proposition 3.1 not requiring such high accuracy, the much simpler second bound in (i) may suffice.

Theorem 3.1. Let $A, B$ and $T$ be finite subsets of an abelian group $G$, with $|A| \geq|B|>k \geq 1$ and $|A| \geq|T|$, such that $r_{A,-B}(x) \leq k$ for all $x \in G \backslash T$. Then then the following bounds hold:

(i) $|A+B| \geq \frac{|A|^{2}|B|^{2}}{M+|A||B|} \geq \frac{|A|^{2}|B|}{|T|(|B|-k)+k|A|}$,

(ii) $|T| \geq \frac{|A|^{2}|B|^{2}-\delta_{0}^{2}-|A+B|\left(k|A||B|-\delta_{0}-\delta(k-\delta)\right)}{|A+B||B|(|B|-k)} \geq \frac{|A|^{2}|B|^{2}-\delta_{0}^{2}-|A+B|\left(k|A||B|-\delta_{0}\right)}{|A+B||B|(|B|-k)} \geq|A| \frac{|A||B|-k|A+B|}{|A+B|(|B|-k)}$,

(iii) $|A+B| \geq \frac{2|A||B|}{\left\lfloor\frac{M+2|||| B \mid}{|A||B|}\right\rfloor}-\frac{M}{\left\lfloor\frac{M+|A||B|}{|A||B|}\right\rfloor\left\lfloor\frac{M+2|A||B|}{|A||B|}\right\rfloor}=\frac{|A|^{2}|B|^{2}(M+2 x)}{(M+|A||B|+x)(M+x)}$,

where $\delta$ is the integer such that $|B|(|A|-|T|) \equiv \delta \bmod k$ with $0 \leq \delta<k$, where $M=|T||B|(|B|-k)+(k-$ $1)|A||B|-\delta(k-\delta)$, where $x$ is the integer, $1 \leq x \leq|A||B|$, such that $M+x \equiv 0 \bmod |A||B|$, and where $\delta_{0}$ is the integer such that $|A||B|+\delta_{0} \equiv 0 \bmod |A+B|$ with $0 \leq \delta_{0}<|A+B|$.

Given a pair of nonempty subsets $(A, B)$ of an abelian group $G$, we can define a graph $\mathcal{M}(A, B)$ whose vertices are the ordered pairs from $A \times B$, with $\left\{(a, b),\left(a^{\prime}, b^{\prime}\right)\right\}$ an edge precisely when $a+b=a^{\prime}+b^{\prime}$. Hence $\mathcal{M}(A, B)$ is a vertex disjoint union of cliques $C l_{x_{i}}$, with each clique $C l_{x_{i}}$ corresponding to an element $x_{i} \in A+B$ such that $r_{A, B}\left(x_{i}\right)=\left|V\left(C l_{x_{i}}\right)\right|=m_{i}$. Letting $n_{i}$ be the number of cliques $C l_{x}$ with $\left|V\left(C l_{x}\right)\right|=i$, 
we note that

$$
\begin{gathered}
|A+B|=\sum_{i \geq 1} n_{i}, \\
|E(\mathcal{M}(A, B))|=\sum_{i \geq 1} n_{i}\left(\begin{array}{l}
i \\
2
\end{array}\right) .
\end{gathered}
$$

The sumset and difference set of $A$ and $B$ are related via these graphs as follows:

$$
|E(\mathcal{M}(A, B))|=|E(\mathcal{M}(A,-B))|
$$

which is easily seen by noting that the map $\varphi: E(\mathcal{M}(A, B)) \rightarrow E(\mathcal{M}(A,-B))$, defined by $\left\{(a, b),\left(a^{\prime}, b^{\prime}\right)\right\} \mapsto$ $\left\{\left(a,-b^{\prime}\right),\left(a^{\prime},-b\right)\right\}$, is bijective (in fact, its own inverse). We remark that it has subsequently come to our attention that the quantity $|E(\mathcal{M}(A, B))|$ is roughly equivalent to the notion of additive energy, for which the reader can consult the text [38].

It is important to note, by means of a simple extremal argument or discrete derivative, that once $|A+B|$ is fixed, then (6) is minimized by taking all cliques of as near equal a size as possible. Likewise, (6) is maximized in just the opposite way, by taking as many cliques of largest allowed size as possible, followed by as many cliques of the next largest allowed size as possible, ..., etc.

Proof. Let $|A|=a,|B|=b$ and $|T|=t$. Since $r_{A,-B}(x) \leq k$ for all $x \in G \backslash T$, since $a \geq b$, and since $a \geq t$, it follows that

$$
|E(\mathcal{M}(A,-B))| \leq t\left(\begin{array}{l}
b \\
2
\end{array}\right)+\frac{b(a-t)-\delta}{k}\left(\begin{array}{l}
k \\
2
\end{array}\right)+\left(\begin{array}{l}
\delta \\
2
\end{array}\right)=\frac{t b^{2}-t k b+(k-1) a b-\delta(k-\delta)}{2}=\frac{M}{2} .
$$

Thus $M \geq 0$ and $M$ is even. Let $l=\left\lceil\frac{a b}{|A+B|}\right\rceil-1$, and let $c=|A+B|$. Note

$$
c=|A+B|=\frac{a b+\delta_{0}}{l+1},
$$

where $a b+\delta_{0} \equiv 0 \bmod c$, and $0 \leq \delta_{0}<c$. Since $|E(\mathcal{M}(A, B))|$ will be minimized when $l \leq r_{A, B}(x) \leq l+1$, it follows in view of (9) that

$$
|E(\mathcal{M}(A, B))| \geq c\left(\begin{array}{l}
l \\
2
\end{array}\right)+(a b-c l) l=\frac{l(2 a b-c(l+1))}{2}=\frac{l\left(a b-\delta_{0}\right)}{2}=\left(\frac{a b+\delta_{0}}{c}-1\right) \frac{\left(a b-\delta_{0}\right)}{2} .
$$

Considering the above bound as a function of $\delta_{0}$, we note that it is quadratic in $\delta_{0}$ with negative lead coefficient. Thus this quantity will be minimized at a boundary value for $\delta_{0}$. Hence comparing the bound evaluated at $\delta_{0}=0$ and $\delta_{0}=c-1$, it follows that

$$
|E(\mathcal{M}(A, B))| \geq\left(\frac{a b}{c}-1\right) \frac{a b}{2} .
$$

Since $|E(\mathcal{M}(A, B))|=|E(\mathcal{M}(A,-B))|$ follows from (7), then by comparing (8) and (10), it follows that $c=|A+B|$ must satisfy the bound

$$
|A+B| \geq \frac{a^{2} b^{2}-\delta_{0}^{2}}{M+a b-\delta_{0}}
$$

and by comparing (8) and (11), it follows that

$$
|A+B| \geq \frac{a^{2} b^{2}}{M+a b}
$$

Noting that $M=t b(b-k)+(k-1) a b-\delta(k-\delta) \leq t b(b-k)+(k-1) a b$, it follows that (13) implies (i), and (12) rearranges to yield (ii) (the second two inequalities follow immediately from the first). 
Suppose that

$$
|A+B| \geq \min \left\{\frac{|A||B|}{d}, \frac{2|A||B|}{d+1}-\frac{M}{d(d+1)}\right\}
$$

for every integer $d \geq 1$. Comparing the two bounds in the minimum, we see that

$$
\frac{2|A||B|}{d+1}-\frac{M}{d(d+1)} \leq \frac{|A||B|}{d}
$$

holds for $d \leq \frac{M+a b}{a b}$. Thus, since $M \geq 0$, then applying (14) with $d=\left\lfloor\frac{M+a b}{a b}\right\rfloor \geq 1$ yields (iii). So it remains to establish (14).

Note that if $l=0$, then $|A+B|=|A||B|$ follows from (9), yielding (14). So we may assume $l>0$. Hence comparing (8) with (10) (expressed in terms of $l$ without $\delta_{0}$ ), it follows that

$$
|A+B| \geq \frac{2 a b}{l+1}-\frac{M}{l(l+1)}
$$

Considering the above bound as a function of $l$, and computing its discrete derivative, i.e., the bound evaluated at $l$ minus the bound evaluated at $l-1$, for $l \geq 2$, we obtain

$$
\frac{2 M+2 a b-2 a b l}{l\left(l^{2}-1\right)}
$$

Noting that (16) is non-negative for $l \leq \frac{M+a b}{a b}$, it follows that the bound in (15) monotonically increases up to $l \leq \frac{M+a b}{a b}$.

Suppose $l>\frac{M+a b}{a b}$. Hence from (9) it follows that

$$
\frac{M+a b+1}{a b} \leq l=\frac{a b+\delta_{0}}{c}-1 \leq \frac{a b+c-1}{c}-1<\frac{a b}{c} .
$$

Thus, in view of (i), it follows that

$$
\frac{a^{2} b^{2}}{M+a b} \leq c<\frac{a^{2} b^{2}}{M+a b+1}
$$

a contradiction. So $l \leq \frac{M+a b}{a b}$. Consequently, the bound in (15) is monotonically increasing for all possible values of $l$.

If $l \leq d-1$, then $|A+B|=c \geq \frac{a b}{d}$ follows from (9). Otherwise, $l \geq d \geq 1$, whence the monotonicity of (15) implies

$$
|A+B| \geq \frac{2 a b}{d+1}-\frac{M}{d(d+1)}
$$

Hence $|A+B|$ is greater than the minimum of these two bounds, yielding (14), and completing the proof.

If $|B| \leq k$, then the restriction $r_{A,-B}(x) \leq k$ for all $x \in G \backslash T$ holds trivially. Hence the assumption $|B|>k$ is needed to gain useful information. Likewise, the assumption $|A| \geq|T|$ is not very restrictive, since for $|A| \leq|T|$ it is possible that $r_{A,-B}(x)=|B|$ for all $x \in A-B$, in which case we obtain only the trivial bound for $\mid E(\mathcal{M}(A,-B) \mid$ (in such cases, an improved estimate of $\mid E(\mathcal{M}(A,-B) \mid$ would yield an improved estimate for $|A+B|$, though we will handle this issue by bounding $|T|$ instead). We remark that the bound given in (iii) is minimized when $x=|A||B|$, which yields (i). However, the estimate $\frac{|A|^{2}|B|^{2}}{M+|A||B|}$ can be improved upon, under a variety of circumstances, by using more precise estimates for $x$. For instance, if $0 \leq|T||B|(|B|-k)-\delta(k-\delta)<|A||B|$, then $x=|A||B|-|T||B|(|B|-k)+\delta(k-\delta)$ follows from the definitions of $M$ and $x$. 


\section{A Step Beyond KST}

We begin by defining four more types (V)-(VIII), which will be the additional basic building blocks, along with the four types of elementary pairs, for constructing all pairs $A$ and $B$ with $|A+B|=|A|+|B|$. We say the pair of nonempty, finite subsets $(A, B)$ of the abelian group $G$ is of type:

(V) if $\min \{|A|,|B|\}=2$ and $|A+B|=|A|+|B|$;

(VI) if $|A|=|B|=3, A=x+B$ for some $x \in G$, and $|A+B|=|A|+|B|$;

(VII) if $(A, B)$ is a dual of a type (VI) pair with respect to some finite $K \leq G$;

(VIII) if there exists a subgroup $K \cong \mathbb{Z} / 2 \mathbb{Z} \times \mathbb{Z} / 2 \mathbb{Z}$ such that $\left(\phi_{K}(A), \phi_{K}(B)\right)$ is of type (II), $A, B$ and $A+B$ are aperiodic, and $d^{\subseteq}(A, A+K)=d^{\subseteq}(B, B+K)=4$ with each of the 4 end terms of $\phi_{K}(A)$ and $\phi_{K}(B)$ containing exactly $2 K$-holes.

Note that $|A+B|=|A|+|B|$ in all cases. In more geometric terms, a type (V) pair consists of a set with precisely two elements whose difference is (say) $d$, paired with another set which is a union of two (but not one) aperiodic arithmetic progressions with difference $d$ along with (possibly) a $\langle d\rangle$-periodic subset. Since $|x+2 A| \leq 2|A|$ for all cardinality three subsets $A \subseteq G$, the restriction $|A+B|=|A|+|B|$ in the description of type (VI) is provided to exclude critical pairs covered by KST.

We now proceed with our main result, Theorem 4.1. Note that the second part of Theorem 4.1 shows that the case with $A+B$ periodic reduces to the case when $A+B$ is aperiodic. In the case when $|G|$ is prime, then the reason for assuming the second part of the bound in (1) was to exclude the cases when $|B|$ is too small to gain exceptional structural information. Indeed, $|A+B| \leq|A||B| \leq|A|+|B|+r$ holds trivially for any pair $(A, B)$ with $\max \{|A|,|B|\}$ sufficiently small compared to $r$. We note that each of the exceptional cases given by types (V)-(VII) correspond precisely to those degenerate cases in $\mathbb{Z} / p \mathbb{Z}$ when $\min \{|A|,|B|\}$ is very small, lifted via a quasi-periodic decomposition in precisely the same way the elementary pairs of types (I)-(IV) were lifted for KST. However, one should note that when only $\min \{|A|,|B|\}$, as opposed to $\max \{|A|,|B|\}$, is small in comparison to $r$, we still gain important structural information about the pair $(A, B)$, though this structure is in general weaker. The last additional exception given by type (VIII) is the first case where a non-quasi-periodic example does not directly correspond to behavior in $\mathbb{Z} / p \mathbb{Z}$. However, the structure of a type (VIII) pair is highly restricted, including $d^{\subseteq}(A+B, \mathcal{P}), d \subseteq(\bar{A}, \mathcal{P}), d \subseteq(\bar{B}, \mathcal{P}) \leq 2$, and cannot occur in a cyclic group.

The degenerate cases in $G=\mathbb{Z} / p \mathbb{Z}$ that correspond to when $A+B$ is very close to $G$ (for instance, type (IV) pairs), also have corresponding analogs whenever $d^{\subseteq}(A+B, \mathcal{P})$ is very small. All this leads one to wonder if $|A+B|=|A|+|B|+r$ and $d^{\subseteq}(\bar{A}, \mathcal{P}), d^{\subseteq}(\bar{B}, \mathcal{P}), d^{\subseteq}(A+B, \mathcal{P}) \geq r+3$, with equality holding for at most one of the three quantities (note that $|A| \geq d^{\subseteq}(\bar{A}, \mathcal{P})$, with equality holding when $|G|$ is prime), would always imply there exists a pair $\left(A^{\prime}, B^{\prime}\right)$ with $\left|A^{\prime}+B^{\prime}\right|=\left|A^{\prime}\right|+\left|B^{\prime}\right|+r^{\prime},-1 \leq r^{\prime}<r$ and $d \subseteq\left(A, A^{\prime}\right), d^{\subseteq}\left(B, B^{\prime}\right) \leq r-r^{\prime}$.

Theorem 4.1. Let $G$ be an abelian group and let $A, B \subseteq G$ be finite and nonempty with $|A+B|=|A|+|B|$. If $A+B$ is aperiodic, then either there exist $\alpha, \beta \in G$ such that

$$
|(A \cup\{\alpha\})+(B \cup\{\beta\})|=|A \cup\{\alpha\}|+|B \cup\{\beta\}|-1,
$$

or there exist quasi-periodic decompositions $A=A_{1} \cup A_{0}$ and $B=B_{1} \cup B_{0}$ with common quasi-period $H$ and $A_{0}$ and $B_{0}$ nonempty such that:

(i) $\phi_{H}\left(A_{0}\right)+\phi_{H}\left(B_{0}\right)$ is a unique expression element in $\phi_{H}(A)+\phi_{H}(B)$;

(ii) $\left|\phi_{H}(A)+\phi_{H}(B)\right|=\left|\phi_{H}(A)\right|+\left|\phi_{H}(B)\right|-1$;

(iii) the pair $\left(A_{0}, B_{0}\right)$ is of type (V), (VI), (VII) or (VIII). 
If $A+B$ is periodic with $H=H(A+B)$, then either (17) holds, or else $A$ and $B$ are $H$-periodic, $\phi_{H}(A+B)$ is aperiodic, and $\left|\phi_{H}(A)+\phi_{H}(B)\right|=\left|\phi_{H}(A)\right|+\left|\phi_{H}(B)\right|$.

A simple coset counting argument shows that if the second part of Theorem 4.1 holds with $\left(A_{0}, B_{0}\right)$ of type (VII) and quasi-period $H$, then the second part also holds with $\left(A_{0}, B_{0}\right)$ of type (VII) and quasi-period $K$, where $K$ is the subgroup from the definition of (VII), and so $H=K$ can be assumed if convenient

Observe that (17) implies a pair $(A, B)$ with $|A+B|=|A|+|B|$ is a large subset of a critical pair $\left(A^{\prime}, B^{\prime}\right)$ (at most one hole in each set). One might also like to know where such holes $\alpha$ and $\beta$ can be placed in the critical pair $\left(A^{\prime}, B^{\prime}\right)$. If the pair $(A, B)$ is extendible, then this question is easily answered using KST as follows. The extendibility of $(A, B)$ implies that (17) holds with $A^{\prime}+B^{\prime}=A+B$, i.e, that only one element $\alpha$ was deleted, say from $A^{\prime}$ (i.e., $\beta \in B$ ), whence there cannot have been any unique expression element of the form $\alpha+b$ in $A^{\prime}+B^{\prime}$. If $r_{A^{\prime}, B^{\prime}}(x) \geq 2$ for all $x$, then $\alpha$ can be any element. Thus let $A^{\prime}=A_{1}^{\prime} \cup A_{0}^{\prime}$ and $B^{\prime}=B_{1}^{\prime} \cup B_{0}^{\prime}$ be the Kemperman decompositions with common quasi-period $H$. In view of KST(iii), $\alpha \in A$ can be any element from $A_{1}^{\prime}$. If we have type (I), then $\alpha \notin A_{0}^{\prime}$; if type (II), then $\alpha$ can be any element in $A_{0}^{\prime}$ except the two end terms of the arithmetic progression; if type (III), then $\alpha$ can be any element other than $a \in A$, where $a+b$, with $b \in B$, is the only unique expression element in $A+B$; and if type (IV), then $\alpha$ can be any element of $A_{0}^{\prime}$ (as there are no unique expression elements in this case). If the pair $(A, B)$ is non-extendible, then the answer is more complicated, and is provided by the following corollary.

Corollary 4.2. Let $A$ and $B$ be finite, nonempty subsets of an abelian group $G$ with $|A+B|=|A|+|B|$, and suppose that (17) holds. Let $A^{\prime}=A \cup\{\alpha\}$ and let $B^{\prime}=B \cup\{\beta\}$. If $(A, B)$ is non-extendible, then $A^{\prime}+B^{\prime}$ cannot be periodic without a unique expression element. Hence let $A^{\prime}=A_{1}^{\prime} \cup A_{0}^{\prime}$ and $B^{\prime}=B_{1}^{\prime} \cup B_{0}^{\prime}$ be the Kemperman decompositions with common quasi-period $H$. Furthermore, one of the following must also hold:

(A) $\left(A^{\prime}, B^{\prime}\right)$ has type (II) with both $\alpha \in A_{0}^{\prime}$ and $\beta \in B_{0}^{\prime}$, both $\alpha$ and $\beta$ are the second term (by appropriate choice of sign for the progression) in their arithmetic progression with difference $d,\left|A_{0}^{\prime}\right|,\left|B_{0}^{\prime}\right| \geq 3$ with equality holding for at most one of the two, and $|\langle d\rangle| \geq\left|A_{0}^{\prime}\right|+\left|B_{0}^{\prime}\right|-1$;

(B) there exist quasi-periodic decompositions $A=A_{1} \cup A_{0}$ and $B=B_{1} \cup B_{0}$ with common, finite quasiperiod $H$ and $A_{0}$ and $B_{0}$ nonempty such that Theorem 4.1(i)(ii) holds and $\left(A_{0}, B_{0}\right)$ is a dual of a type (V) pair with respect to $H$;

(C) $\left(A^{\prime}, B^{\prime}\right)$ has type (I) such that both the aperiodic parts in the Kemperman decompositions have cardinality one, $\alpha \in A_{1}^{\prime}, \beta \in B_{1}^{\prime}$, and $\left(\phi_{H}\left(A^{\prime}\right), \phi_{H}\left(B^{\prime}\right)\right)$ has type (II) with both $\phi_{H}\left(A_{\alpha}^{\prime}\right)$ and $\phi_{H}\left(B_{\beta}^{\prime}\right)$ contained in the aperiodic part of the mod $H$ Kemperman decomposition, and if $|H|>2$, then both are the second term in their arithmetic progression with $A_{0}^{\prime}+\beta=B_{0}^{\prime}+\alpha=\gamma \notin A+B$, while if $|H|=2$, then either both are the first term in their progression, and $A_{0}^{\prime}+\beta \neq B_{0}^{\prime}+\alpha$ if both progressions have length two, or else both are the second term in their arithmetic progression, $A_{0}^{\prime}+\beta=B_{0}^{\prime}+\alpha=\gamma \notin A+B$, and one of the progressions has at least three terms (in all cases, by appropriate choice of sign for the progressions).

Proof. Since $(A, B)$ is non-extendible and (17) holds, it follows that $\left(A^{\prime}+B^{\prime}\right) \backslash \gamma=A+B$, with $\gamma \in A^{\prime}+B^{\prime}$. Furthermore, we may assume that $\left|N_{1}^{\beta}\left(A^{\prime}, B^{\prime}\right)\right|=\left|N_{1}^{\alpha}\left(B^{\prime}, A^{\prime}\right)\right|=0$, since otherwise the non-extendibility of $(A, B)$ is contradicted by either the critical pair $\left(A^{\prime}, B\right)$ or the critical pair $\left(A, B^{\prime}\right)$. Thus $\gamma$ must have exactly two distinct representations $\alpha+\beta^{\prime}$ and $\alpha^{\prime}+\beta$ in $A^{\prime}+B^{\prime}$.

Suppose $\alpha \in A_{0}^{\prime}$ and $\beta \in B_{0}^{\prime}$ (we assume for the moment that $A_{1}^{\prime} \cup A_{0}^{\prime}$ and $B_{1}^{\prime} \cup B_{0}^{\prime}$ exist, which will be shown to be the case in a following paragraph by similar arguments), and let $A_{0}=A_{0}^{\prime} \backslash \alpha$ and $B_{0}=B_{0}^{\prime} \backslash \beta$. Hence, since $\left|N_{1}^{\beta}\left(A^{\prime}, B^{\prime}\right)\right|=\left|N_{1}^{\alpha}\left(B^{\prime}, A^{\prime}\right)\right|=0$, it follows that $\left(A^{\prime}, B^{\prime}\right)$ cannot have type (I). If we have type (II), then $A_{0}^{\prime}$ and $B_{0}^{\prime}$ are arithmetic progressions with common difference $d$, and by choosing an appropriate 
sign for $d$, it follows that both $\alpha$ and $\beta$ must be the second term in their respective progression. Furthermore, $\left|N_{1}^{\beta}\left(A^{\prime}, B^{\prime}\right)\right|=\left|N_{1}^{\alpha}\left(B^{\prime}, A^{\prime}\right)\right|=0$ implies that $\left|A_{0}^{\prime}\right|,\left|B_{0}^{\prime}\right| \geq 3$, and KST implies that $|\langle d\rangle| \geq\left|A_{0}^{\prime}\right|+\left|B_{0}^{\prime}\right|-1$. Additionally, $\left|A_{0}^{\prime}\right|=\left|B_{0}^{\prime}\right|=3$ cannot hold, else $\left|\left(A^{\prime}+B^{\prime}\right) \backslash(A+B)\right| \geq 2$, a contradiction. Thus (A) holds.

If we have type (IV), then $\left|A_{0}+B_{0}\right|=|H|-2$, whence in view of Proposition 2.4 we see that (B) follows. If we have type (III), then $d \subseteq(A+B, \mathcal{P})=1$ and $\left|A_{0}\right|+\left|B_{0}\right|=|H|-1$, with both $A_{0}$ and $B_{0}$ contained in an $H$-coset. Note that there are exactly $|H|-\left|B_{0}\right|$ elements $x \in A_{0}+H$ such that $\gamma \notin x+B_{0}$. Since $\left|A_{0}\right|<|H|-\left|B_{0}\right|$, it follows that there must be at least one such $x \in \overline{A_{0}} \cap\left(A_{0}+H\right)$, whence adding $x$ contradicts the non-extendibility of $(A, B)$. Thus type (III) cannot occur for $\left(A^{\prime}, B^{\prime}\right)$ in this situation.

Next suppose $A^{\prime}+B^{\prime}$ is periodic without a unique expression element. Thus $(A+B) \cup\{\gamma\}$ is periodic with maximal period (say) $K$, and there are exactly $|K|+1$ holes in $A$ and $B$. Since $\gamma \notin A+B$, it follows in view of Proposition 2.1 applied with $G=K$ that there must exist $a_{0} \in A$ and $b_{0} \in B$ such that $A_{a_{0}}$ and $B_{b_{0}}$ contain at least $|K|$ holes with $\gamma \in A_{a_{0}}+B_{b_{0}}+K$. Thus if the last remaining hole is not also in either $A_{a_{0}}$ or $B_{b_{0}}$, then adding it to either $A$ or $B$ will contradict the non-extendibility of $(A, B)$. Hence $\left|A_{a_{0}}\right|+\left|B_{b_{0}}\right|=|K|-1$, whence the arguments used in the previous paragraph for type (III) show that the pair $(A, B)$ is extendible. Thus $A^{\prime}+B^{\prime}$ cannot be periodic without a unique expression element. Consequently, in view of previous cases, we may suppose $\alpha \in A_{1}^{\prime}$ or $\beta \in B_{1}^{\prime}$, and w.l.o.g. assume the former.

Suppose $|H|=2$. Thus $d^{\subseteq}(A+B, \mathcal{P}) \leq 2$ and either $\left|A_{0}^{\prime}\right|=1$ or $\left|B_{0}^{\prime}\right|=1$. Hence $\left(A^{\prime}, B^{\prime}\right)$ has type (I), whence $\left|N_{1}^{\beta}\left(A^{\prime}, B^{\prime}\right)\right|=0$ implies $\beta \in B_{1}^{\prime}$ as well. Since $\gamma$ has exactly two representations in $A^{\prime}+B^{\prime}$, since every element of $A_{0}^{\prime}+B_{0}^{\prime}$ is a unique expression element, and since $\phi_{H}\left(A_{0}^{\prime}\right)+\phi_{H}\left(B_{0}^{\prime}\right)$ is a unique expression element, it follows that $\phi_{H}\left(A_{0}^{\prime}\right)+\phi_{H}\left(B_{0}^{\prime}\right) \neq \phi_{H}(\gamma)$. Hence, since $\gamma \notin A+B$, it follows that $\phi_{H}\left(A_{0}^{\prime}\right)+\phi_{H}\left(B_{\beta}^{\prime}\right)$, $\phi_{H}\left(B_{0}^{\prime}\right)+\phi_{H}\left(A_{\alpha}^{\prime}\right)$, and $\phi_{H}\left(A_{\alpha}^{\prime}\right)+\phi_{H}\left(B_{\beta}^{\prime}\right)$ are the only possible representations of $\phi_{H}(\gamma) \in \phi_{H}\left(A^{\prime}\right)+\phi_{H}\left(B^{\prime}\right)$.

Suppose $\phi_{H}\left(A_{\alpha}^{\prime}\right)+\phi_{H}\left(B_{\beta}^{\prime}\right)$ is a unique expression element. Hence, since $\phi_{H}\left(A_{0}^{\prime}\right)+\phi_{H}\left(B_{0}^{\prime}\right)$ is also a unique expression element, and since $\alpha \notin A_{0}^{\prime}$ and $\beta \notin B_{0}^{\prime}$, it follows that $\left(\phi_{H}\left(A^{\prime}\right), \phi_{H}\left(B^{\prime}\right)\right)$ must have type (II) with (by choosing the appropriate sign for the progression) $\phi_{H}\left(A_{0}^{\prime}\right)$ the first term in the arithmetic progression and $\phi_{H}\left(A_{\alpha}^{\prime}\right)$ the last term in the arithmetic progression, and the same holding true for $\phi_{H}\left(B_{0}^{\prime}\right)$ and $\phi_{H}\left(B_{\beta}^{\prime}\right)$. Since $\phi_{H}\left(A_{\alpha}^{\prime}\right)+\phi_{H}\left(B_{\beta}^{\prime}\right)$ is a unique expression element, and since $|H|=2$, it follows that $\left(A_{\alpha}^{\prime} \backslash \alpha\right)+\left(B_{\beta}^{\prime} \backslash \beta\right)$ must be missing an element from the coset $\alpha+\beta+H$, which must then be $\gamma$ (since deleting $\alpha$ and $\beta$ removes only the single element $\gamma$ from $\left.A^{\prime}+B^{\prime}\right)$. Hence $\phi_{H}\left(A_{\alpha}^{\prime}\right)+\phi_{H}\left(B_{\beta}^{\prime}\right)=\phi_{H}(\gamma)$. Thus, since $\phi_{H}\left(A_{\alpha}^{\prime}\right)+\phi_{H}\left(B_{\beta}^{\prime}\right)=\phi_{H}(\gamma)$ is a unique expression element, it follows that $\left|A_{0}^{\prime}\right|=\left|B_{0}^{\prime}\right|=1$, else adding the other element from the $H$-coset either to $A_{0}^{\prime}$ or $B_{0}^{\prime}$ will contradict the non-extendibility of $(A, B)$. Furthermore, if there are only two terms in each progression, then it follows that $\left(A_{0}^{\prime}+\left(B_{\beta}^{\prime} \backslash \beta\right)\right) \cup\left(B_{0}^{\prime}+\left(A_{\alpha}^{\prime} \backslash \alpha\right)\right)$ is an $H$-coset, else $A+B$ will be missing an element besides $\gamma$ from the coset $A_{0}^{\prime}+B_{\beta}^{\prime}+H=B_{0}^{\prime}+A_{\alpha}^{\prime}+H$, contradicting that $\left(A^{\prime} \backslash \alpha\right)+\left(B^{\prime} \backslash \beta\right)=\left(A^{\prime}+B^{\prime}\right) \backslash \gamma$. Hence $A_{0}^{\prime}+\beta \neq B_{0}^{\prime}+\alpha$ in this case. Thus (C) holds. So next assume $\phi_{H}\left(A_{\alpha}^{\prime}\right)+\phi_{H}\left(B_{\beta}^{\prime}\right)$ is not a unique expression element.

If $\phi_{H}\left(A_{\alpha}^{\prime}\right)+\phi_{H}\left(B_{\beta}^{\prime}\right)=\phi_{H}(\gamma)$, then there will be two representations of $\gamma$ contained in $A_{\alpha}^{\prime}+B_{\beta}^{\prime}$ (since $\alpha \in A_{1}^{\prime}$ and $\left.\beta \in B_{1}^{\prime}\right)$, whence $\phi_{H}\left(A_{\alpha}^{\prime}\right)+\phi_{H}\left(B_{\beta}^{\prime}\right)$ not a unique expression element implies that $\gamma$ must have at least one more representation in $A^{\prime}+B^{\prime}$ ( since $\phi_{H}\left(A_{0}^{\prime}\right)+\phi_{H}\left(B_{0}^{\prime}\right)$ is a unique expression element, and hence not equal to $\phi_{H}\left(A_{\alpha}^{\prime}\right)+\phi_{H}\left(B_{\beta}^{\prime}\right)=\phi_{H}(\gamma)$, and since all other pairs $\phi_{H}\left(b_{1}\right) \in \phi_{H}\left(A^{\prime}\right)$ and $\phi_{H}\left(b_{2}\right) \in \phi_{H}\left(B^{\prime}\right)$ have either $b_{1}+H \subseteq A^{\prime}$ or $\left.b_{2}+H \subseteq B^{\prime}\right)$, a contradiction. Therefore $\phi_{H}\left(A_{\alpha}^{\prime}\right)+\phi_{H}\left(B_{\beta}^{\prime}\right) \neq \phi_{H}(\gamma)$.

Thus, since $\gamma$ has exactly two representations in $A^{\prime}+B^{\prime}$ given by $\alpha+\beta^{\prime}$ and $\alpha^{\prime}+\beta$, and since $\phi_{H}\left(A_{0}^{\prime}\right)+$ $\phi_{H}\left(B_{\beta}^{\prime}\right), \phi_{H}\left(B_{0}^{\prime}\right)+\phi_{H}\left(A_{\alpha}^{\prime}\right)$, and $\phi_{H}\left(A_{\alpha}^{\prime}\right)+\phi_{H}\left(B_{\beta}^{\prime}\right)$ are the only possible representations of $\phi_{H}(\gamma) \in \phi_{H}\left(A^{\prime}\right)+$ $\phi_{H}\left(B^{\prime}\right)$, it follows that $\phi_{H}\left(A_{0}^{\prime}\right)+\phi_{H}\left(B_{\beta}^{\prime}\right)=\phi_{H}\left(B_{0}^{\prime}\right)+\phi_{H}\left(A_{\alpha}^{\prime}\right)=\phi_{H}(\gamma)$ is an element of $\phi_{H}\left(A^{\prime}\right)+\phi_{H}\left(B^{\prime}\right)$ with exactly two representations, and that $\left|A_{0}^{\prime}\right|=\left|B_{0}^{\prime}\right|=1$. From KST it follows, as remarked in Section 2, 
that if $\left(A^{\prime}, B^{\prime}\right)$ has type (I) with $\left|A_{0}^{\prime}\right|=1$, then $\left(\phi_{H}\left(A^{\prime}\right), \phi_{H}\left(B^{\prime}\right)\right)$ cannot have type (I) with the aperiodic part of $\phi_{H}\left(A^{\prime}\right)$ having cardinality one. Likewise if $\left|B_{0}^{\prime}\right|=1$.

Let $L / H$ be the quasi-period from KST applied modulo $H$ to $\left(\phi_{H}\left(A^{\prime}\right), \phi_{H}\left(B^{\prime}\right)\right)$. Then in view of the previous paragraph, we conclude that $\left|\left(A_{0}^{\prime}+L\right) \cap A\right| \geq 3$ and $\left|\left(B_{0}^{\prime}+L\right) \cap B\right| \geq 3$ (recall $\phi_{H}\left(A_{0}+L\right)$ and $\phi_{H}\left(B_{0}+L\right)$ are, in view of KST(iii), the aperiodic parts of the Kemperman decompositions modulo $H$ ). Consequently, since $\alpha^{\prime}+\beta=\alpha+\beta^{\prime}=\gamma$ is an element with precisely two representations in $A+B$, we must have both $\phi_{H}\left(B_{\beta}^{\prime}\right)$ and $\phi_{H}\left(A_{\alpha}^{\prime}\right)$ contained in the $L / H$-coset corresponding to the aperiodic part of their Kemperman decomposition (i.e., $B_{\beta}^{\prime} \subseteq B_{0}^{\prime}+L$ and $A_{\alpha}^{\prime} \subseteq A_{0}^{\prime}+L$ ).

If $\left(\phi_{H}\left(A^{\prime}\right), \phi_{H}\left(B^{\prime}\right)\right)$ has type (II), then (by appropriate choice of $\operatorname{sign}$ ), $\phi_{H}\left(A_{0}^{\prime}\right)$ is the first term in the arithmetic progression, and $\phi_{H}\left(A_{\alpha}^{\prime}\right)$ is the second term, with the same true of $\phi_{H}\left(B_{0}^{\prime}\right)$ and $\phi_{H}\left(B_{\beta}^{\prime}\right)$ (in view of the previous paragraph, and since $\phi_{H}\left(A_{\alpha}^{\prime}\right)+\phi_{H}\left(B_{0}^{\prime}\right)=\phi_{H}\left(A_{0}^{\prime}\right)+\phi_{H}\left(B_{\beta}^{\prime}\right)$ is an element with exactly two distinct representations in $\left.\phi_{H}\left(A^{\prime}\right)+\phi_{H}\left(B^{\prime}\right)\right)$. Furthermore, $\phi_{H}\left(A_{\alpha}^{\prime}\right)+\phi_{H}\left(B_{\beta}^{\prime}\right)$ not a unique expression element implies that $\phi_{H}\left(A_{\alpha}^{\prime}\right)$ and $\phi_{H}\left(B_{\beta}^{\prime}\right)$ cannot also both be end terms, whence there must be at least three terms in one of the progressions. Additionally, since $\phi_{H}\left(B_{0}^{\prime}\right)+\phi_{H}\left(A_{\alpha}^{\prime}\right)=\phi_{H}\left(A_{0}^{\prime}\right)+\phi_{H}\left(B_{\beta}^{\prime}\right)=\phi_{H}(\gamma)$, it follows that $\gamma \notin\left(A_{0}^{\prime}+\left(B_{\beta}^{\prime} \backslash \beta\right)\right) \cup\left(B_{0}^{\prime}+\left(A_{\alpha}^{\prime} \backslash \alpha\right)\right)$, whence $A_{0}^{\prime}+\beta=B_{0}^{\prime}+\alpha=\gamma$. Thus (C) holds.

Note that $\left(\phi_{H}\left(A^{\prime}\right), \phi_{H}\left(B^{\prime}\right)\right)$ cannot have type (IV), nor, as we remarked earlier, type (I). If instead $\left(\phi_{H}\left(A^{\prime}\right), \phi_{H}\left(B^{\prime}\right)\right)$ has type (III), then $\phi_{H}\left(A_{0}^{\prime}\right)+\phi_{H}\left(B_{\beta}^{\prime}\right)=\phi_{H}\left(B_{0}^{\prime}\right)+\phi_{H}\left(A_{\alpha}^{\prime}\right)$ being an element of $\phi_{H}\left(A^{\prime}\right)+$ $\phi_{H}\left(B^{\prime}\right)$ with exactly two representations would imply $\phi_{H}\left(A_{0}^{\prime}\right)+\phi_{H}\left(B_{\beta}^{\prime}\right)$ was a unique expression element in $\phi_{H}\left(A^{\prime}\right)+\phi_{H}\left(B^{\prime} \backslash B_{0}^{\prime}\right)$. Recall that for a type (III) pair each aperiodic part has at size at least three, i.e., $\left|\phi_{H}\left(\left(A_{0}^{\prime}+L\right) \cap A\right)\right|,\left|\phi_{H}\left(\left(B_{0}^{\prime}+L\right) \cap B\right)\right| \geq 3$. Hence, since $\phi_{H}\left(A_{0}^{\prime}\right)+\phi_{H}\left(B_{0}^{\prime}\right)$ is the unique expression element in $\phi_{H}\left(A^{\prime}\right)+\phi_{H}\left(B^{\prime}\right)$, it follows from KST that $\left(\phi_{H}\left(A^{\prime}\right), \phi_{H}\left(B^{\prime} \backslash B_{0}^{\prime}\right)\right)$ either has type (IV), whence there cannot be any unique expression element, a contradiction, or else type (II). In the latter case, then $\phi_{H}\left(A_{0}^{\prime}\right)+\phi_{H}\left(B_{\beta}^{\prime}\right)$ a unique expression element in $\left(\phi_{H}\left(A^{\prime}\right)+\phi_{H}\left(B^{\prime} \backslash B_{0}^{\prime}\right)\right)$ implies that $\phi_{H}\left(A_{0}^{\prime}\right)$ is an end term of the arithmetic progression, whence $\phi_{H}\left(A_{0}^{\prime}\right)+\phi_{H}\left(B_{0}^{\prime}\right)$ a unique expression element in $\phi_{H}\left(A^{\prime}\right)+\phi_{H}\left(B^{\prime}\right)$ implies that $\phi_{H}\left(B_{0}^{\prime}\right)$ must follow directly after/before the end term of the progression $\phi_{H}\left(B^{\prime} \backslash B_{0}^{\prime}\right)$, whence $\left(\phi_{H}\left(A^{\prime}\right), \phi_{H}\left(B^{\prime}\right)\right)$ has type (II), a contradiction. Thus type (III) cannot occur for $\left(\phi_{H}\left(A^{\prime}\right), \phi_{H}\left(B^{\prime}\right)\right)$ in this situation.

Finally, assume instead $|H|>2$. Since $\alpha \in A_{1}^{\prime}$, then from Proposition 2.1, it follows that $\left(A_{\alpha}^{\prime} \backslash \alpha\right)+B_{b_{i}}$ will be $H$-periodic for all $\left|B_{b_{i}}\right| \geq 2$. Thus $\gamma \notin A+B$ and $|H|>2$ imply that $\phi_{H}\left(A_{\alpha}^{\prime}\right)+\phi_{H}\left(B_{0}^{\prime}\right)=\phi_{H}(\gamma)$, and that either $\left|B_{0}^{\prime}\right|=1$ or else $\left|B_{0}^{\prime}\right|=2$ and $\beta \in B_{0}^{\prime}$. However, in the latter case, $\left|B_{0}^{\prime}\right|=2$ implies that type (I) or (II) must hold (since type (III) and (IV) both imply that each of the aperiodic parts of the Kemperman decompositions have cardinality at least three), whence $\left|B_{0}^{\prime}\right|=2$ further implies that $\left|N_{1}^{b}(A, B)\right|>0$ for all $b \in B_{0}^{\prime}$, contradicting that $\left|N_{1}^{\beta}(A, B)\right|=0$. Therefore we can assume $\left|B_{0}\right|=1$, whence we must have type (I). Hence $\left|N_{1}^{\beta}(A, B)\right|=0$ implies that $\beta \in B_{1}$ also. Applying the same arguments with the roles of $A$ and $B$ reversed, it follows that $\left|B_{0}\right|=\left|A_{0}\right|=1$ with $\phi_{H}\left(A_{0}^{\prime}\right)+\phi_{H}\left(B_{\beta}^{\prime}\right)=\phi_{H}\left(B_{0}^{\prime}\right)+\phi_{H}\left(A_{\alpha}^{\prime}\right)=\phi_{H}(\gamma)$ an element of $\phi_{H}\left(A^{\prime}+B^{\prime}\right)$ with exactly two representations (since $r_{A^{\prime}, B^{\prime}}(\gamma)=2$ ), whence the arguments from the previous three paragraphs apply for determining the structure modulo $H$, with the exception that the arithmetic progressions from KST are allowed to both have length two, yielding (C), and completing the proof.

We remark that the sufficiency of the examples (A)-(C) is easily checked, as is the sufficiency for types (V)(VIII) (in view of Proposition 2.1, Lemma 5.3, and KST). Thus together, along with the description provided for extendible pairs $(A, B)$, they may be taken as necessary and sufficient conditions for $|A+B|=|A|+|B|$. Simple consequences of the above description and Theorem 4.1 are the following two immediate corollaries. 
Corollary 4.3. Let $A$ and $B$ be finite, nonempty, and non-extendible subsets of an abelian group $G$ such that $|A+B|=|A|+|B|$. If $-a+A$ and $-b+B$, where $a \in A$ and $b \in B$, are non-quasi-periodic, generating subsets such that $|A|,|B|,|\overline{A+B}| \geq 3$, with equality holding for at most one of the three, then

$$
\begin{gathered}
d^{\subseteq}(A+B, \mathcal{S})=d^{\subseteq}(\overline{A+B}, \mathcal{S})=1, \text { and } \\
d^{\subseteq}(B, \mathcal{S})=d^{\subseteq}(\bar{B}, \mathcal{S})=d^{\subseteq}(A, \mathcal{S})=d^{\subseteq}(\bar{A}, \mathcal{S})=1,
\end{gathered}
$$

where either $\mathcal{S}=\mathcal{Q} \mathcal{P}_{H}$, for a nontrivial, proper subgroup $H$, or else $\mathcal{S}=\mathcal{A} \mathcal{P}_{d}$, for a nonzero $d \in G$.

Corollary 4.4. Let $A$ and $B$ be finite, nonempty subsets of an abelian group $G$ such that $|A+B|=|A|+|B|$ and $d \subseteq(A, \mathcal{Q P}), d^{\subseteq}(B, \mathcal{Q P}) \geq 2$. If $d \subseteq(\bar{A}, \mathcal{P}), d^{\subseteq}(\bar{B}, \mathcal{P}), d^{\subseteq}(A+B, \mathcal{P}) \geq 3$, with equality holding for at most one of the three, then for some nonzero $d \in G$,

$$
d^{\subseteq}\left(A+B, \mathcal{A} \mathcal{P}_{d}\right), d^{\subseteq}\left(A, \mathcal{A} \mathcal{P}_{d}\right), d^{\subseteq}\left(B, \mathcal{A} \mathcal{P}_{d}\right) \leq 1
$$

\section{Preliminary Lemmas}

The proof of Theorem 4.1 is heavily reliant upon a series of reductions to simpler cases. An important step in the proof will be to show that it suffices to prove Theorem 4.1 when both $A$ and $B$ are non-quasi-periodic, generating subsets. This will be accomplished principally through the following three lemmas.

Lemma 5.1. Let $A$ and $B$ be finite, nonempty subsets of an abelian group $G$ with $|A| \geq 3,|A+B|=|A|+|B|$, and $0 \in A$, and let $H=\langle A\rangle$. If $A+B$ is aperiodic and $B$ is non-extendible, then $B$ has a quasi-periodic decomposition with quasi-period $H$.

Proof. Let $B^{\prime}$ be the maximal subset of $B$ that is $H$-periodic, and let $B \backslash B^{\prime}=B_{b_{1}} \cup \ldots \cup B_{b_{l}}$ be an $H$-coset decomposition of $B \backslash B^{\prime}$. From the maximality of $B^{\prime}$ it follows that no $B_{b_{i}}$ is $H$-periodic. Hence, since $B$ is non-extendible, it follows that

$$
A+B_{b_{i}} \neq b_{i}+H
$$

for all $i$. Since $|A+B|=|A|+|B|$, since $B^{\prime}$ is $H$-periodic, and since $\left|\phi_{H}(A)\right|=1$, it follows that

$$
\left|A+\left(B \backslash B^{\prime}\right)\right|=|A|+\left|B \backslash B^{\prime}\right|
$$

If the lemma is false, then $l \geq 2$. Thus

$$
\left|A+\left(B \backslash B^{\prime}\right)\right|=\left|\cup_{i=1}^{l} A+B_{b_{i}}\right| \geq\left|A+B_{b_{1}}\right|+\left|A+B_{b_{2}}\right|+\left|B \backslash\left\{B^{\prime} \cup B_{b_{1}} \cup B_{b_{2}}\right\}\right|,
$$

implying in view of (19) that

$$
\left|A+B_{b_{1}}\right|+\left|A+B_{b_{2}}\right|=\left|A+\left(B_{b_{1}} \cup B_{b_{2}}\right)\right| \leq|A|+\left|B_{b_{1}}\right|+\left|B_{b_{2}}\right| .
$$

In view of Kneser's Theorem, it follows that

$$
\left|A+B_{b_{i}}\right| \geq|A|+\left|B_{b_{i}}\right|-\left|H_{i}\right|+\rho_{i},
$$

where $A+B_{b_{i}}$ is maximally $H_{i}$-periodic and $\rho_{i}$ is the number of $H_{i}$-holes in $A$ and $B_{b_{i}}$. Since $H=\langle A\rangle$, it follows in view of $(18)$ that $\left|\phi_{H_{i}}(A)\right| \geq 2$, whence

$$
|A| \geq\left|\phi_{H_{i}}(A)\right|\left|H_{i}\right|-\rho_{i} \geq 2\left|H_{i}\right|-\rho_{i} .
$$

Combining (21) and (20), and w.l.o.g. assuming $\left|H_{1}\right| \geq\left|H_{2}\right|$, it follows that

$$
|A| \leq\left|H_{1}\right|+\left|H_{2}\right|-\rho_{1}-\rho_{2} \leq 2\left|H_{1}\right|-\rho_{1}-\rho_{2} .
$$


Thus in view of (22) applied with $i=1$, it follows that $\rho_{2}=0$. Hence, since $A$ is aperiodic (else $A+B$ is periodic), it follows that $\left|H_{2}\right|=1$, whence (23) and (22) imply $\left|H_{1}\right|=1$ also. Thus (23) implies $|A| \leq 2$, a final contradiction.

Lemma 5.2. Let $A$ and $B$ be finite, nonempty subsets of an abelian group $G$ with $|A+B|=|A|+|B|$, $0 \in A \cap B$, and $|A|,|B| \geq 3$. If $A+B$ is aperiodic, $(A, B)$ is non-extendible, and neither $A$ nor $B$ is quasi-periodic, then $\langle A\rangle=\langle B\rangle$.

Proof. Since $A$ is not quasi-periodic, it follows from Lemma 5.1 that $\langle A\rangle \leq\langle B\rangle$. Likewise $\langle B\rangle \leq\langle A\rangle$, whence $\langle A\rangle=\langle B\rangle$.

Lemma 5.3. Let $A$ and $B$ be finite, nonempty subsets of an abelian group $G$ with $A+B$ aperiodic and $(A, B)$ non-extendible, and let $A=A_{1} \cup A_{0}$ be a quasi-periodic decomposition with $A_{1}$ nonempty and periodic with maximal period $H$. If $|A+B|=|A|+|B|$, then $B$ has a quasi-periodic decomposition $B=B_{1} \cup B_{0}$ with quasi-period $H$, such that:

(i) $\phi_{H}\left(A_{0}\right)+\phi_{H}\left(B_{0}\right)$ is a unique expression element in $\phi_{H}(A)+\phi_{H}(B)$,

(ii) $\left|\phi_{H}(A+B)\right|=\left|\phi_{H}(A)\right|+\left|\phi_{H}(B)\right|-1$,

(iii) $\left|A_{0}+B_{0}\right|=\left|A_{0}\right|+\left|B_{0}\right|$.

Proof. Let $B^{\prime}$ be the maximal subset of $B$ that is $H$-periodic, and let $B \backslash B^{\prime}=B_{b_{1}} \cup \ldots \cup B_{b_{l}}$ be an $H$-coset decomposition of $B \backslash B^{\prime}$. From the maximality of $B^{\prime}$ it follows that no $B_{b_{i}}$ is $H$-periodic. Hence, since $B$ is non-extendible, it follows, for all $i$, that

$$
A_{0}+B_{b_{i}} \neq b_{i}+H
$$

If $\left|\phi_{H}\left(A_{1}\right)+\phi_{H}(B)\right|<\left|\phi_{H}\left(A_{1}\right)\right|+\left|\phi_{H}(B)\right|-1$, then from Kneser's Theorem it follows that $\phi_{H}\left(A_{1}\right)+\phi_{H}(B)$ is periodic, contradicting either the maximality of $H$ for $A_{1}$ or the fact that $A$ is non-extendible. Therefore

$$
\left|\phi_{H}\left(A_{1}\right)+\phi_{H}(B)\right| \geq\left|\phi_{H}\left(A_{1}\right)\right|+\left|\phi_{H}(B)\right|-1 \text {. }
$$

Since $A+B$ is aperiodic, it follows that $|H|\left|\phi_{H}(B)\right|>|B|$ and that $|A+B| \geq\left|A_{1}+B\right|+\left|A_{0}\right|$. Hence if (25) is strict, then

$$
|A+B| \geq|H|\left(\left|\phi_{H}\left(A_{1}\right)\right|+\left|\phi_{H}(B)\right|\right)+\left|A_{0}\right| \geq|A|+|B|+1
$$

a contradiction. Therefore we can assume

$$
\left|\phi_{H}\left(A_{1}\right)+\phi_{H}(B)\right|=\left|\phi_{H}\left(A_{1}\right)\right|+\left|\phi_{H}(B)\right|-1 \text {. }
$$

Likewise, if $A_{0}+B^{\prime} \nsubseteq A_{1}+B$, then $|A+B| \geq\left|A_{1}+B\right|+|H|+\left|A_{0}\right|$, whence (26) again follows, a contradiction. So we may assume otherwise.

From the non-extendibility of $B$, and in view of (24), it follows that $A_{0}+B_{b_{i}}$ is disjoint from $A_{1}+B$ for all $i$. Thus $\phi_{H}\left(A_{0}\right)+\phi_{H}\left(B_{b_{i}}\right)$ is a unique expression element for each $i$. Hence, since $A_{0}+B^{\prime} \subseteq A_{1}+B$ and noting that $\left|\phi_{H}\left(A_{1}\right)\right|=\left|\phi_{H}(A)\right|-1$, it follows in view of $(27)$ that

$$
\left|\phi_{H}(A+B)\right|=\left|\phi_{H}(A)\right|+\left|\phi_{H}(B)\right|-2+l .
$$

Note (i) and (ii) along with $|A+B|=|A|+|B|$ force (iii) by a simple counting argument. Consequently, the proof will be complete if $l=1$. So assume $l \geq 2$.

From Kneser's Theorem applied via translation with group $H$, it follows that

$$
\left|A_{0}+B_{b_{i}}\right| \geq\left|A_{0}\right|+\left|B_{b_{i}}\right|-\left|H_{i}\right|
$$


where $A_{0}+B_{b_{i}}$ is maximally $H_{i}$-periodic, and $H_{i} \leq H$. In view of (27), and since each $A_{0}+B_{b_{i}}$ is disjoint from $A_{1}+B$, it follows that

$$
\begin{gathered}
|A+B| \geq\left(\left|\phi_{H}\left(A_{1}\right)\right|+\left|\phi_{H}\left(B^{\prime}\right)\right|+l-1\right)|H|+\sum_{i=1}^{l}\left|A_{0}+B_{b_{i}}\right|= \\
|A|+\left|B^{\prime}\right|-\left|A_{0}\right|+(l-1)|H|+\sum_{i=1}^{l}\left|A_{0}+B_{b_{i}}\right| \geq \\
|A|+|B|-\left|A_{0}\right|-\left|B_{b_{1}}\right|-\left|B_{b_{2}}\right|+(l-1)|H|+\left|A_{0}+B_{b_{1}}\right|+\left|A_{0}+B_{b_{2}}\right| .
\end{gathered}
$$

In view of (24), it follows that $\left|H_{i}\right| \leq \frac{1}{2}|H|$. Thus (29), (30) and $l \geq 2$ together imply that

$$
|A+B| \geq|A|+|B|+\left|A_{0}\right|
$$

whence $\left|A_{0}\right|=0$ and $A=A_{1}$, contradicting that $A+B$, and thus $A$, is aperiodic.

The following lemma essentially shows that it is sufficient for $A$ to be a non-quasi-periodic, generating subset in order that this be true (at least when $G$ is finite) of every $C \in\{A, B, A+B, \bar{B}, \bar{A}, \overline{A+B}\}$. In the proof, we will often reduce the case $A+B=C$ to a case $A^{\prime}+B^{\prime}=C^{\prime}$, where at least one of $A^{\prime} B^{\prime}$ and $C^{\prime}$ is a set from $\pm\{A, B, A+B, \bar{B}, \bar{A}, \overline{A+B}\}$, and then employ an induction hypothesis to $A^{\prime}+B^{\prime}=C^{\prime}$. However, since we will want to stay restricted to the class of non-quasi-periodic, generating subsets, the following lemma, along with Proposition 2.4, will allow us to transfer these assumptions from $A+B=C$ to $A^{\prime}+B^{\prime}=C^{\prime}$.

Lemma 5.4. Let $A$ and $B$ be finite, nonempty subsets of an abelian group $G$ with $|A+B|=|A|+|B|$, $0 \in A \cap B,|A|,|B|,|\overline{A+B}| \geq 3, A+B$ aperiodic and $(A, B)$ non-extendible. If $\langle A\rangle=G$ and $A$ is not quasi-periodic, then $B$ is not quasi-periodic and $\langle B\rangle=G$. Furthermore, if $G$ is finite, then neither $A+B$ nor $\overline{A+B}$ is quasi-periodic, and $\langle-\gamma+\overline{A+B}\rangle=G$, where $\gamma \in \overline{A+B}$.

Proof. If $B$ is quasi-periodic with quasi-period $H$, then since $A$ is not quasi-periodic, it follows in view of Lemma 5.3 that $G=\langle A\rangle \leq H$, implying $H=G$. Hence $B=G$, contradicting that $A+B$ is aperiodic. Therefore we can assume $B$ is not quasi-periodic. Hence from Lemma 5.2 it follows that $G=\langle A\rangle=\langle B\rangle$.

Now assume $G$ is finite. Since $(A, B)$ is non-extendible, it follows in view of Proposition 2.4 that $-A+$ $\overline{A+B}=\bar{B}$, with $(-A, \overline{A+B})$ non-extendible. Since $A+B$ is aperiodic, it follows that $B$, and thus $\bar{B}$, is aperiodic. Hence from the result of the previous paragraph applied to $-A$ and $\overline{A+B}$, it follows that $\overline{A+B}$ is not quasi-periodic and $\langle-\gamma+\overline{A+B}\rangle=\langle A\rangle=G$, where $\gamma \in \overline{A+B}$. If $A+B$ is quasi-periodic with quasi-period $H$, then it follows, in view of $\overline{A+B}$ not quasi-periodic, that $G=\langle-\gamma+\overline{A+B}\rangle \leq H$. Hence $H=G$, contradicting that $A+B$ is aperiodic, and completing the proof.

In the proof, once we have established that Theorem 4.1 holds for $A^{\prime}+B^{\prime}=C^{\prime}$, we will want to transfer the resulting structure back to $A+B=C$. Since one of $A^{\prime}, B^{\prime}$ and $C^{\prime}$ will be a set from $\pm\{A, B, A+$ $B, \bar{A}, \bar{B}, \overline{A+B}\}$, our strategy will be to use this common linking set (along with Proposition 2.4) as the means of transferring the structural information. However, to accomplish this, we will need to know that only having the unpaired structural information for the set $A$ is enough to conclude that Theorem 4.1 holds for the pair that includes $A$. The following two lemmas accomplish this in the case when a non-quasiperiodic, generating subset $A$ is close to being quasi-periodic. Note that we have begun assuming $G$ finite in the hypotheses of the lemmas. This is done to make use of the set $\overline{A+B}$ via Proposition 2.4. Since we will be able to later show that the case $G$ infinite follows from the case $G$ finite, this will not hinder the proof. However, we first prove the following simple proposition that will be needed in several places. 
Proposition 5.5. Let $A$ and $B$ be finite subsets of an abelian group, and let $C \subseteq A+B$. Suppose $|A|,|B|,|C| \geq 2$. If $(a+B) \cap C$ and $(b+A) \cap C$ are both nonempty for every $a \in A$ and $b \in B$, then either (a) there exist distinct $a, a^{\prime} \in A$, distinct $b, b^{\prime} \in B$ and distinct $c, c^{\prime} \in C$ such that $a+b=c$ and $a^{\prime}+b^{\prime}=c^{\prime}$, or else (b) $|A|=|B|=|C|=2$, and there exists $a \in A$ and $b \in B$ such that $a+B=b+A=C$.

Proof. Consider the edge colored bipartite graph $\Gamma$ with partition classes $A \times\{0\}$ and $B \times\{1\}$ and an edge joining $(a, 0)$ and $(b, 1)$ colored $c$ whenever $a+b=c \in C$. Then (a) is equivalent to finding two disjoint edges with distinct colors. The condition that $C$ intersects $a+B$ and $A+b$ for each $a \in A$ and $b \in B$ translates to the fact that $\Gamma$ has no isolated vertices.

Take the set of edges with colors $c, c^{\prime} \in C, c \neq c^{\prime}$. We obtain a graph $\Gamma^{\prime}$ composed of two monochromatic matchings. If $\Gamma^{\prime}$ has at least five vertices, then the statement easily follows (the pigeonhole principle guarantees two edges $e_{1}$ and $e_{2}$ of the same color, which must be disjoint, whence an edge from a fifth vertex not in either edge will either give a new edge colored distinctly, yielding (a) together with either $e_{1}$ or $e_{2}$, or else a third parallel edge $e_{3}$ of the same color, in which case any edge colored distinctly in $\Gamma^{\prime}$ with yield (a) with one of $e_{1}, e_{2}$ or $\left.e_{3}\right)$. So assume $\Gamma^{\prime}$ has at most four vertices. If there is vertex in $\Gamma$ but not $\Gamma^{\prime}$, then it must be incident with a third color $c^{\prime \prime} \in C$, and we again get our pair of disjoint edges with different colors (if $\Gamma^{\prime}$ contains only one vertex from either partition class, then make sure to choose the new vertex from the partition class having only one element in $\Gamma^{\prime}$, which is possible in view of $|A|,|B| \geq 2$ ). Otherwise, since $\min \{|A|,|B|\} \geq 2$, we have four vertices in $\Gamma$ and $|A|=|B|=2$. Moreover, it is easily seen that $|C|=2$, else (a) again follows. Consequently, since there are no isolated vertices in $\Gamma=\Gamma^{\prime}$, there must be at least one vertex in $A$ and one vertex in $B$ of degree 2, which gives the remaining part in (b).

Lemma 5.6. Let $A$ and $B$ be nonempty subsets of a finite abelian group $G$ with $|A+B|=|A|+|B|, 0 \in A \cap B$, $|A|,|B|, d^{\subseteq}(A+B, \mathcal{P}) \geq 3,(A, B)$ non-extendible, $\langle A\rangle=G$ and $A$ not quasi-periodic. If $A=A^{\prime} \cup A_{2} \cup A_{1}$ with $A^{\prime}$ a nonempty periodic subset with maximal period $H, A_{1} \subseteq a_{1}+H$, and $A_{2} \subseteq a_{2}+H$, for some $a_{i} \in G$, then $d \subseteq(C, \mathcal{Q P})=1$ for all $C \in\{A, B, A+B, \bar{A}, \bar{B}, \overline{A+B}\}$ and (17) holds.

Proof. Let $B^{\prime}$ be the maximal subset of $B$ that is $H$-periodic, and let $B \backslash B^{\prime}=B_{b_{1}} \cup \ldots \cup B_{b_{l}}$ be an $H$-coset decomposition of $B \backslash B^{\prime}$. From the maximality of $B^{\prime}$ it follows that no $B_{b_{i}}$ is $H$-periodic. From Lemma 5.4 it follows that $\langle B\rangle=G$ and that $B$ is not quasi-periodic. Hence $l \geq 2$, as otherwise $H=G$ implying $A=G$, which contradicts $A+B$ aperiodic. Since $A$ is not quasi-periodic, it follows that $A_{1}$ and $A_{2}$ are both nonempty partially filled $H$-cosets that are disjoint modulo $H$.

If $\left|\phi_{H}\left(A^{\prime}\right)+\phi_{H}(B)\right|<\left|\phi_{H}\left(A^{\prime}\right)\right|+\left|\phi_{H}(B)\right|-1$, then from Kneser's Theorem it follows that $\phi_{H}\left(A^{\prime}\right)+\phi_{H}(B)$ is periodic, contradicting either the maximality of $H$ for $A^{\prime}$ or the fact that $A$ is non-extendible. Therefore

$$
\left|\phi_{H}\left(A^{\prime}\right)+\phi_{H}(B)\right| \geq\left|\phi_{H}\left(A^{\prime}\right)\right|+\left|\phi_{H}(B)\right|-1 .
$$

Let $C^{\prime}$ be the maximal subset of $A+B$ that is $H$-periodic, and let $(A+B) \backslash C^{\prime}=C_{c_{1}} \cup C_{c_{2}} \cup \ldots \cup C_{c_{r}}$ be an $H$-coset decomposition. In view of Lemma 5.4 it follows that $A+B$ is not quasi-periodic. Thus $r \geq 2$ (as $A^{\prime} \neq \emptyset$ implies $C^{\prime} \neq \emptyset$ ). Note that $A^{\prime}+B \subseteq C^{\prime}$. Hence from (31) it follows that

$$
\begin{aligned}
& |A+B| \geq\left(\left|\phi_{H}\left(A^{\prime}\right)\right|+\left|\phi_{H}\left(B^{\prime}\right)\right|+l-1\right)|H|+\sum_{i=1}^{r}\left|C_{c_{i}}\right| \geq \\
& |A|+|B|-\left(\left|A_{1}\right|+\left|A_{2}\right|+\sum_{i=1}^{l}\left|B_{b_{i}}\right|\right)+(l-1)|H|+\sum_{i=1}^{r}\left|C_{c_{i}}\right| .
\end{aligned}
$$

Since the $C_{c_{i}}$ are partially filled $H$-cosets, it follows that $\phi_{H}\left(C \backslash C^{\prime}\right) \subseteq \phi_{H}\left(A_{1} \cup A_{2}\right)+\phi_{H}\left(B \backslash B^{\prime}\right)$. From the non-extendibility of $A$, and since each $A_{i}$ is a partially filled $H$-coset, it follows that each $a_{i}$ has at least one $b_{j}$ 
such that $\phi_{H}\left(a_{i}\right)+\phi_{H}\left(b_{j}\right) \in \phi_{H}\left(C \backslash C^{\prime}\right)$. In view of the non-extendibility of $B$, and since each $B_{b_{i}}$ is a partially filled $H$-coset, it follows that same holds true for each $b_{i}$. Consequently, it follows in view of Proposition 5.5 that either there exist distinct $b_{i_{1}}$ and $b_{i_{2}}$, and distinct $c_{i_{1}^{\prime}}$ and $c_{i_{2}^{\prime}}$, such that $\phi_{H}\left(a_{1}+b_{i_{1}}\right)=\phi_{H}\left(c_{i_{1}^{\prime}}\right)$ and $\phi_{H}\left(a_{2}+b_{i_{2}}\right)=\phi_{H}\left(c_{i_{2}^{\prime}}\right)$, or else $l=r=2$ and w.l.o.g. $\phi_{H}\left(a_{1}+\left\{b_{1}, b_{2}\right\}\right)=\left\{\phi_{H}\left(c_{1}\right), \phi_{H}\left(c_{2}\right)\right\}$ and $\phi_{H}\left(b_{1}+\left\{a_{1}, a_{2}\right\}\right)=\left\{\phi_{H}\left(c_{1}\right), \phi_{H}\left(c_{2}\right)\right\}$. We handle these cases separately.

Case 1: First assume there exist distinct $b_{i_{1}}$ and $b_{i_{2}}$, and distinct $c_{i_{1}^{\prime}}$ and $c_{i_{2}^{\prime}}$, such that $\phi_{H}\left(a_{1}+b_{i_{1}}\right)=$ $\phi_{H}\left(c_{i_{1}^{\prime}}\right)$ and $\phi_{H}\left(a_{2}+b_{i_{2}}\right)=\phi_{H}\left(c_{i_{2}^{\prime}}\right)$. Since the $C_{c_{i}}$ are partially filled, it follows from Kneser's Theorem that $\left|A_{1}+B_{b_{i_{1}}}\right| \geq\left|A_{1}\right|+\left|B_{b_{i_{1}}}\right|-\left|H_{1}\right|+\rho_{1}$, for some proper subgroup $H_{1}<H$, where $\rho_{1}$ is the number of $H_{1}$-holes in $A_{1}$ and $B_{b_{i_{1}}}$. Likewise $\left|A_{2}+B_{b_{i_{2}}}\right| \geq\left|A_{2}\right|+\left|B_{b_{i_{2}}}\right|-\left|H_{2}\right|+\rho_{2}$. Hence, since $\left|H_{i}\right| \leq \frac{1}{2}|H|$, and since $|H|>\left|B_{b_{i}}\right|$ for all $i$, it follows in view of (32) that

$$
\begin{gathered}
|A+B| \geq|A|+|B|-\left(\left|A_{1}\right|+\left|A_{2}\right|+\sum_{i=1}^{l}\left|B_{b_{i}}\right|\right)+(l-1)|H|+\sum_{i=1}^{r}\left|C_{c_{i}}\right| \geq \\
|A|+|B|-\left(\left|A_{1}\right|+\left|A_{2}\right|+\left|B_{i_{1}}\right|+\left|B_{i_{2}}\right|\right)+|H|+\left|A_{1}+B_{b_{i_{1}}}\right|+\left|A_{2}+B_{b_{i_{2}}}\right| \geq \\
|A|+|B|+|H|-\left|H_{1}\right|-\left|H_{2}\right|+\rho_{1}+\rho_{2} \geq|A|+|B| .
\end{gathered}
$$

Hence, since $|A+B|=|A|+|B|$, it follows that $r=l=2, \rho_{1}=\rho_{2}=0$, and $\left|H_{1}\right|=\left|H_{2}\right|=\frac{1}{2}|H|$, as otherwise the above estimate will be strict.

Since $r=2$, then $|H|=2$ would imply $d \subseteq(A+B, \mathcal{P}) \leq 2$, contrary to our hypothesis. Therefore $\left|H_{1}\right|=\left|H_{2}\right|=\frac{1}{2}|H|>1$. If $H_{1} \cap H_{2}$ is nontrivial, then in view of $\rho_{1}=\rho_{2}=0$, it follows that $A$, and thus $A+B$, is periodic, a contradiction. Thus it follows that $|H| \geq\left|H_{1}\right|\left|H_{2}\right|=\frac{1}{4}|H|^{2}$. Hence, since $\left|H_{1}\right|=\left|H_{2}\right|=\frac{1}{2}|H|>1$, it follows that $|H|=4$. Thus $d^{\subseteq}\left(A+B, A+B+H_{1}\right) \leq|H|-\left|H_{2}\right|=2$, contradicting that $d^{\subseteq}(A+B, \mathcal{P}) \geq 3$, and completing the case.

Case 2: Next assume that $l=r=2$, and that w.l.o.g. $\phi_{H}\left(a_{1}+\left\{b_{1}, b_{2}\right\}\right)=\phi_{H}\left(b_{1}+\left\{a_{1}, a_{2}\right\}\right)=$ $\left\{\phi_{H}\left(c_{1}\right), \phi_{H}\left(c_{2}\right)\right\}$. Hence, since each $C_{c_{i}}$ is a partially filled $H$-coset, it follows in view of Proposition 2.1 that

$$
\left|A_{1}\right|+\left|B_{b_{2}}\right| \leq|H| \text { and }\left|B_{b_{1}}\right|+\left|A_{2}\right| \leq|H|
$$

Since $\phi_{H}\left(b_{1}+\left\{a_{1}, a_{2}\right\}\right)=\left\{\phi_{H}\left(c_{1}\right), \phi_{H}\left(c_{2}\right)\right\}$, it follows that $\left|C_{c_{1}}\right|+\left|C_{c_{2}}\right| \geq\left|A_{2}\right|+\left|B_{b_{1}}\right|$, and from Kneser's Theorem applied to $B_{b_{1}}+A_{1}$, it follows that equality is possible only if $C_{c_{1}} \cup C_{c_{2}}=B_{b_{1}}+\left(A_{1} \cup A_{2}\right)$ is $K$-periodic with $\phi_{K}\left(A_{1}\right)=1$ and $K \leq H$. Thus, since $A+B$ is aperiodic, it follows that equality is possible only if $\left|A_{1}\right|=1$. The same argument applied to $\phi_{H}\left(a_{1}+\left\{b_{1}, b_{2}\right\}\right)=\left\{\phi_{H}\left(c_{1}\right), \phi_{H}\left(c_{2}\right)\right\}$ also shows that $\left|C_{c_{1}}\right|+\left|C_{c_{2}}\right| \geq\left|B_{b_{2}}\right|+\left|A_{1}\right|$, with equality possible only if $\left|B_{b_{1}}\right|=1$. Hence, since $|A+B| \leq|A|+|B|$, it follows in view of (34) and (32), that we must in fact have equality in both the estimates $\left|C_{c_{1}}\right|+\left|C_{c_{2}}\right| \geq\left|B_{b_{2}}\right|+\left|A_{1}\right|$ and $\left|C_{c_{1}}\right|+\left|C_{c_{2}}\right| \geq\left|A_{2}\right|+\left|B_{b_{1}}\right|$, as well as both inequalities in (34). Consequently, $\left|A_{1}\right|=\left|B_{b_{1}}\right|=1$, $\left|A_{2}\right|=\left|B_{b_{2}}\right|=|H|-1$, and $C_{c_{1}} \cup C_{c_{2}}=B_{b_{1}}+\left(A_{1} \cup A_{2}\right)$. Thus in view of Lemma 5.4, it follows that $d^{\subseteq}\left(C, \mathcal{Q P}_{H}\right)=d^{\subseteq}(C, \mathcal{Q P})=1$ for all $C \in\{A, B, A+B, \bar{A}, \bar{B}, \overline{A+B}\}$. Hence, since $d^{\subseteq}(A+B, \mathcal{P}) \geq 3$, it follows that $|H| \geq 3$. Thus, since the $C_{c_{i}}$ are partially filled, and since $C_{c_{1}} \cup C_{c_{2}}=B_{b_{1}}+\left(A_{1} \cup A_{2}\right)$, it follows in view of Proposition 2.1 that $\phi_{H}\left(a_{2}+b_{2}\right) \neq \phi_{H}\left(c_{i}\right)$ for all $i$, and that $\phi_{H}\left(a_{2}+b_{1}\right)=\phi_{H}\left(a_{1}+b_{2}\right)$. Consequently, letting $\alpha$ be the $H$-hole in $A_{2}$, and letting $\beta$ be the $H$-hole in $B_{b_{2}}$, it follows that $|(A \cup\{\alpha\})+(B \cup\{\beta\})|=$ $|A+B|+1=|A \cup\{\alpha\}|+|B \cup\{\beta\}|-1$, yielding (17), and completing the proof.

Lemma 5.7. Let $A$ and $B$ be nonempty subsets of a finite abelian group $G$ with $|A+B|=|A|+|B|$, $0 \in A \cap B,|A|,|B|, d^{\subseteq}(A+B, \mathcal{P}) \geq 3,(A, B)$ non-extendible, and $\langle A\rangle=G$. If $d^{\subseteq}(A, \mathcal{Q P})=1$ and either $|A| \geq 4$ or $|B| \geq 4$, then $d^{\subseteq}(C, \mathcal{Q P})=1$ for all $C \in\{A, B, A+B, \bar{A}, \bar{B}, \overline{A+B}\}$ and (17) holds. 
Proof. In view of Lemma 5.6, it follows the proof is complete unless $A=A_{1} \cup A_{0}$ with each $A_{i}$ a subset of an $H$-coset and $\left|A_{1}\right|=|H|-1$, for some nontrivial subgroup $H$. If $A_{0}$ is empty, then $\langle A\rangle=G$ implies that $H=G$, whence $|A|=|G|-1$, contradicting $d^{\subseteq}(A+B, \mathcal{P}) \geq 3$. Therefore we can assume $A_{0}$ is nonempty. Let $b_{1}, \ldots, b_{c}$ be those elements of $B$ that are the unique element from their $H$-coset in $B$, and let $B^{\prime}=B_{b_{1}^{\prime}} \cup \ldots \cup B_{b_{l}^{\prime}}$ be an $H$-coset decomposition of the remaining elements of $B$. Since $A$ is not quasi-periodic it follows that $\left|A_{0}\right|<|H|$. Hence $|A| \geq 3$ implies $|H| \geq 3$. In view of Lemma 5.4, it follows that $B$ is not quasi-periodic and $\langle B\rangle=G$. We divide the proof into two cases.

Case 1. Suppose $\left|\phi_{H}(A)+\phi_{H}(B)\right| \geq\left|\phi_{H}(A)\right|+\left|\phi_{H}(B)\right|-1=\left|\phi_{H}(B)\right|+1$. Since $\left|A_{1}\right|=|H|-1$ and $\left|B_{b_{i}^{\prime}}\right|>1$, it follows in view of Proposition 2.1 and the assumption of the case that

$$
\begin{aligned}
|A+B| \geq \mid & \left|A_{1}+B\right|+\left|A_{0}\right| \geq(l+c)|H|-c+\left|A_{0}\right|= \\
& \left(|H|+\left|A_{0}\right|-1\right)+(l|H|+c)+c(|H|-2)-|H|+1 \geq|A|+|B|+\rho^{\prime}+(c-1)(|H|-2)-1,
\end{aligned}
$$

where $\rho^{\prime}$ is the number of $H$-holes in $B^{\prime}$. Hence $|A+B| \leq|A|+|B|$ implies

$$
\rho^{\prime}+(c-1)(|H|-2)-1 \leq 0 .
$$

Note $c \geq 1$, since otherwise adding the $H$-hole contained in $A_{1}$ will, in view of Proposition 2.1, contradict the non-extendibility of $A$.

Suppose $\rho^{\prime}=0$. If $B^{\prime}$ is empty, then $|B| \geq 3$ implies $c \geq 3$; otherwise, the cases $c \leq 2$ are covered by Lemma 5.6. Thus in all cases we can assume $c \geq 3$, whence (35) implies $2|H| \leq 5$, contradicting $|H| \geq 3$. So we can assume $\rho^{\prime}>0$.

If $\rho^{\prime}>1$, then (35) and $|H| \geq 3$ imply $c \leq 0$, a contradiction. Therefore we can assume $\rho^{\prime}=1$, whence (35) and $|H| \geq 3$ imply $c=1$. Hence if $\left|\phi_{H}(B)\right|>2$, then the proof is complete in view of $\rho^{\prime}=1$ and Lemma 5.6. Otherwise $\rho^{\prime}=c=1$ implies that $B=B_{1} \cup B_{0}$ with both $B_{i}$ nonempty subsets of disjoint $H$-cosets, $\left|B_{1}\right|=|H|-1$, and $\left|B_{0}\right|=1$. Thus the hypotheses of the lemma and the case are satisfied by interchanging the roles of $A$ and $B$, whence $\left|A_{0}\right|=1$ follows by the above argument as well. Since $A$ is not quasi-periodic and since $\langle A\rangle=G$, it follows that $\bar{A}$ is not quasi-periodic. Likewise for $B$. Hence $d \subseteq(B, \mathcal{Q P})=d \subseteq(\bar{B}, \mathcal{Q P})=d \subseteq(A, \mathcal{Q P})=d \subseteq(\bar{A}, \mathcal{Q P})=1$. In view of Proposition 2.1 and $|H| \geq 3$, it follows that $\left|A_{1}+B_{1}\right|=|H|$ and $A_{0}+B_{1}=A_{1}+B_{0}$, since otherwise $|A+B| \geq 2|H|+1>|A|+|B|$, a contradiction. Hence $d \subseteq\left(A+B, \mathcal{Q P}_{H}\right)=d \subseteq\left(\overline{A+B}, \mathcal{Q P}_{H}\right)=1$. Thus, since neither $A+B$ nor $\overline{A+B}$ is quasi-periodic (in view of Lemma 5.4), it follows that $d^{\subseteq}(A+B, \mathcal{Q P})=d^{\subseteq}(\overline{A+B}, \mathcal{Q P})=1$. Finally, by letting $\alpha$ be the $H$-hole in $A_{1}$, and by letting $\beta$ be the $H$-hole in $B_{1}$, it follows in view of $A_{1}+B_{0}=A_{0}+B_{1}$ and $\left|A_{1}+B_{1}\right|=|H|$ that $|(A \cup\{\alpha\})+(B \cup\{\beta\})|=|A+B|+1=|A \cup\{\alpha\}|+|B \cup\{\beta\}|-1$, yielding (17), and completing the case.

Case 2. Suppose $\left|\phi_{H}(A)+\phi_{H}(B)\right|=\left|\phi_{H}(B)\right|$. Thus from Kneser's Theorem it follows that $\phi_{H}(B)$ is periodic with maximal subgroup $K / H$, and that $\phi_{H}(A)$ is contained in an $K / H$-coset. Hence, since $\langle A\rangle=G$, it follows that $K=G$ and that $G / H$ is cyclic generated by $\phi_{H}\left(A_{1}\right)-\phi_{H}\left(A_{0}\right)$. Letting $\rho^{\prime}$ be the number of $H$-holes in $B^{\prime}$, it follows in view of Proposition 2.1 that

$$
\begin{array}{r}
|A+B| \geq(l+c)|H|-c=\left(|H|-1+\left|A_{0}\right|\right)+(l|H|+c)+(c-1)(|H|-2)-\left|A_{0}\right|-1 \geq \\
|A|+|B|-\left|A_{0}\right|+\rho^{\prime}+(c-1)(|H|-2)-1,
\end{array}
$$

with equality possible only if $A_{1}+b_{i}+H \nsubseteq A+B$ for $i=1, \ldots, c$. Since $d^{\subseteq}(A+B, \mathcal{P}) \geq 3$, it follows, in view of Proposition 2.1 and the assumption of the case, that $c \geq 3$, with equality possible only if $A_{1}+b_{i}+H \nsubseteq A+B$ for $i=1, \ldots, c$. 
Suppose $l>0$. Hence, since $\phi_{H}\left(A_{1}\right)-\phi_{H}\left(A_{0}\right)$ generates $G / H$, and since $c \geq 3>0$, it follows that there exists $b_{j}^{\prime}$ such that $\phi_{H}\left(A_{0}+b_{j}^{\prime}\right)=\phi_{H}\left(A_{1}+b_{i}\right)$ for some $b_{i}$. Thus it follows in view of Proposition 2.1 that either $\rho^{\prime} \geq\left|A_{0}\right|$, or else $A_{1}+b_{i}+H=A_{0}+b_{j}^{\prime}+H \subseteq A+B$. In the former case, it follows in view of (36), $|A+B| \leq|A|+|B|$ and $|H| \geq 3$, that $c \leq 2$, a contradiction. In the latter case, it follows that the inequality (36) is strict and that $c \geq 4$. Hence it follows, in view of $|A+B| \leq|A|+|B|$ and $\left|A_{0}\right| \leq|H|-1$, that $2|H| \leq 5$, contradicting $|H| \geq 3$. So we may assume $l=0$, whence $c=|B|$.

Suppose $\left|A_{0}\right| \leq|H|-2$. In view of (36), $|A+B| \leq|A|+|B|,|H| \geq 3$, and $c \geq 3$, it follows that $\left|A_{0}\right| \geq 2|H|-5 \geq|H|-2$. Thus $\left|A_{0}\right| \leq|H|-2$ implies $|H|=3$, whence $\left|A_{0}\right| \leq|H|-2$ implies $|A|=3$. Thus by hypothesis $c=|B| \geq 4$, whence (36) implies $\left|A_{0}\right| \geq 3|H|-7 \geq|H|-1$, a contradiction. So we can assume $\left|A_{0}\right|=|H|-1$.

If $A_{1}+b_{i}+H \subseteq A+B$ for some $i$, then (36) will be strict and $c \geq 4$, whence $\left|A_{0}\right| \geq 3|H|-6 \geq|H|$, a contradiction. Therefore we can assume $A_{1}+b_{i}+H \nsubseteq A+B$ for all $i$. Thus, since $\phi_{H}(B)$ is $G / H$-periodic with $G / H$ cyclic generated by $\phi_{H}\left(A_{1}\right)-\phi_{H}\left(A_{0}\right)$, and since $\left|A_{1}\right|=\left|A_{0}\right|=|H|-1$, it follows that we can permute the $b_{i}$ such that $A_{1}+b_{i}=A_{0}+b_{j}$ for $i \equiv j+1 \bmod c$. Consequently,

$$
\begin{aligned}
& A+B+\left\{b_{1}, b_{2}\right\}=\left(\bigcup_{i=1}^{c} A_{1}+b_{i}\right)+\left\{b_{1}, b_{2}\right\}=\left(\bigcup_{i=1}^{c} A_{1}+b_{i}+b_{1}\right) \cup\left(\bigcup_{i=1}^{c} A_{1}+b_{2}+b_{i}\right)= \\
& \left(\bigcup_{i=1}^{c} A_{1}+b_{i}+b_{1}\right) \cup\left(\bigcup_{i=1}^{c} A_{0}+b_{1}+b_{i}\right)=\left(\left(\bigcup_{i=1}^{c} A_{1}+b_{i}\right) \cup\left(\bigcup_{i=1}^{c} A_{0}+b_{i}\right)\right)+b_{1}=A+B+b_{1},
\end{aligned}
$$

implying from Kneser's Theorem that $A+B$ is periodic, a contradiction.

The following lemma will also be used to transfer unpaired structural information from the single set $A$ to a pair containing $A$, this time in the case when $A$ is an arithmetic progression.

Lemma 5.8. Let $A$ and $B$ be finite, nonempty subsets of an abelian group $G$ with $|A+B|=|A|+|B|$, $|A| \geq 3$, and $A+B$ aperiodic. If $A$ is an arithmetic progression with difference $d$, then $d \subseteq\left(B, \mathcal{Q A P} \mathcal{P}_{d}\right)=1$, $d^{\subseteq}\left(A+B, \mathcal{Q A P}_{d}\right)=d^{\subseteq}\left(\overline{A+B}, \mathcal{Q} \mathcal{A} \mathcal{P}_{d}\right)=0$ and (17) holds.

Proof. Note that $|A+B| \geq|B|+c(|A|-1)$, where $c$ is the number of $d$-components of $\bar{B}$ with length at least $|A|-1$. Since $A+B$ is aperiodic, it follows that there must be at least one $d$-component of $\bar{B}$ with length at least $|A|-1$. Hence either $|A+B| \geq|B|+2(|A|-1)$ or else $|A+B|=|B|+|A|-1+d \subseteq\left(B, \mathcal{Q A P}_{d}\right)$. Since $|A| \geq 3$, and since $|A+B|=|A|+|B|$, it follows that the former cannot hold, whence the latter implies $d^{\subseteq}\left(B, \mathcal{Q} \mathcal{A} \mathcal{P}_{d}\right)=1$. Hence, since $|A| \geq 3$, it follows that $d^{\subseteq}\left(A+B, \mathcal{Q} \mathcal{A} \mathcal{P}_{d}\right)=0$, whence $d^{\subseteq}\left(\overline{A+B}, \mathcal{Q} \mathcal{A} \mathcal{P}_{d}\right)=$ 0 as well. Letting $\beta$ be the single hole in $B$ and letting $\alpha \in A$, it follows that (17) holds.

The following lemma will be one of our main tools for reducing the case $A+B=C$ to a case $A^{\prime}+B^{\prime}=C^{\prime}$. Lemma 5.9 will allow us to conclude the sets $A^{\prime}=A+\{0, d\}, B^{\prime}=B$ and $C^{\prime}=C+\{0, d\}$ also satisfy $\left|A^{\prime}+B^{\prime}\right|=\left|A^{\prime}\right|+\left|B^{\prime}\right|$ (whence induction will be employed), provided $c_{d}(A)=2$ for some nonzero $d$. We note this was (more or less) the main strategy used to prove the prime order case of Theorem 4.1 in [19]. Lemma 5.9 will also be needed for Lemma 5.10 .

Lemma 5.9. Let $A$ and $B$ be nonempty subsets of a finite abelian group $G$ with $|A+B|=|A|+|B|, 0 \in A \cap B$, $|A| \geq 4,|B| \geq 3, d \subseteq(A+B, \mathcal{P}) \geq 3,(A, B)$ non-extendible, $\langle A\rangle=G$ and $A$ not quasi-periodic. If $c_{d}(A)=2$ for some nonzero $d$, then either $c_{d}(B), c_{d}(A+B) \leq 2$, or else $(17)$ holds and either $d \subseteq(C, \mathcal{Q P})=1$, for all $C \in\{A, B, A+B, \bar{A}, \bar{B}, \overline{A+B}\}$, or $d \subseteq(B, \mathcal{A P})=0$. 
Proof. Since $A$ is non-extendible, it follows in view of Proposition 2.4 that $-B+\overline{A+B}=\bar{A}$. Suppose $c_{d}(B) \geq 3$. Hence, since $c_{d}(A)=c_{d}(\bar{A})=2$, and since $|A+B|=|A|+|B|$, it follows that

$$
|(-B+\{0, d\})+\overline{A+B}| \leq|-B+\{0, d\}|+|\overline{A+B}|-1,
$$

since otherwise

$$
\begin{aligned}
|G|-|A|+2 & =|\bar{A}|+2=|\{0, d\}+\bar{A}|=|-B+\{0, d\}+\overline{A+B}| \\
& \geq|-B+\{0, d\}|+|\overline{A+B}| \geq|B|+3+|\overline{A+B}|=|G|-|A|+3,
\end{aligned}
$$

which is not possible.

If $\bar{A}+\{0, d\}$ is periodic, then $|A| \geq 3$ and $c_{d}(\bar{A})=2$ imply that $A$ is a union of a nonempty periodic set and at most two elements, whence Lemma 5.6 implies $d \subseteq(A, \mathcal{Q P})=1$. Thus Lemma 5.7 completes the proof. Therefore we can assume that $\bar{A}+\{0, d\}$ is aperiodic. Hence Kneser's Theorem and (37) imply $c_{d}(B)=3$ and $\left.|(-B+\{0, d\})+\overline{A+B}|=\mid-B+\{0, d\}\right)|+| \overline{A+B} \mid-1$, whence we can apply KST to the pair $(-B+\{0, d\}, \overline{A+B})$. Let $-B+\{0, d\}=B_{1} \cup B_{0}$ and $\overline{A+B}=C_{1} \cup C_{0}$ be the Kemperman decompositions with common quasi-period $H$.

In view of Lemma 5.4, it follows that $\overline{A+B}$ is not quasi-periodic and that $\langle\gamma-\overline{A+B}\rangle=G$ for $\gamma \in \overline{A+B}$. Hence $H=G$, whence we cannot have type (I), nor as the sumset is aperiodic can we have type (III).

Suppose we have type (II). Hence $\overline{A+B}$ is an arithmetic progression with difference $d^{\prime}$, whence Lemma 5.8 applied to $(-B, \overline{A+B})$ implies $d^{\subseteq}\left(B, \mathcal{Q} \mathcal{A} \mathcal{P}_{d^{\prime}}\right)=1$ and $d^{\subseteq}\left(\bar{A}, \mathcal{Q} \mathcal{A} \mathcal{P}_{d^{\prime}}\right)=d^{\subseteq}\left(A, \mathcal{Q} \mathcal{A} \mathcal{P}_{d^{\prime}}\right)=0$. However, in view of Proposition 2.4, and Lemma 5.8 applied to $(-A, \overline{A+B})$, it follows that $d \subseteq\left(A, \mathcal{Q A P} \mathcal{P}_{d^{\prime}}\right)=1$, contradicting $d \subseteq\left(A, \mathcal{Q} \mathcal{A} \mathcal{P}_{d^{\prime}}\right)=0$. So we cannot have type (II), and thus must have type (IV). Hence, since $\langle-\gamma+\overline{A+B}\rangle=G$, for $\gamma \in \overline{A+B}$, it follows that $|\bar{A}+\{0, d\}|=|G|-1$, implying $|A| \leq 3$, a contradiction. So we can assume $c_{d}(B) \leq 2$.

Applying the above argument with the roles of $B$ and $\overline{A+B}$ interchanged, we find that either $c_{d}(\overline{A+B})=$ $c_{d}(A+B) \leq 2$, or else $B$ is an arithmetic progression. Hence, since $c_{d}(B) \leq 2$, it follows that we can assume the later case holds, else the proof is complete. Thus in view of Lemma 5.8 it follows that (17) holds, completing the proof.

The next lemma stretches Lemma 5.8 one step further, to handle the case $d^{\subseteq}(A, \mathcal{A P})=1$.

Lemma 5.10. Let $A$ and $B$ be nonempty subsets of a finite abelian group $G$ with $|A+B|=|A|+|B|$, $0 \in A \cap B,|A|,|B|, d^{\subseteq}(A+B, \mathcal{P}) \geq 3$, and $(A, B)$ non-extendible, $\langle A\rangle=G$ and $A$ not quasi-periodic. If $d \subseteq\left(A, \mathcal{A P}_{d}\right)=1$ for some nonzero $d \in G$, and if at most one of $|A|,|B|$ and $|\overline{A+B}|$ is equal to 3 , then (17) holds and one of $d \subseteq(B, \mathcal{Q A \mathcal { P }} d) \leq 1$ or $d^{\subseteq}\left(B, \mathcal{Q A} \mathcal{P}_{d^{\prime}}\right)=0$, for some non-zero $d^{\prime}$, or $d^{\subseteq}(C, \mathcal{Q P})=1$, for all $C \in\{A, B, A+B, \bar{A}, \bar{B}, \overline{A+B}\}$, also holds

Proof. Since $d \subseteq\left(A, \mathcal{A} \mathcal{P}_{d}\right)=1$, it follows that $A$ is a subset of an arithmetic progression with difference $d$ and one hole. Hence, $c_{d}(A)=2$. Furthermore, since $\langle A\rangle=G$, it follows that $\langle d\rangle=G$. Since $B$ is not quasiperiodic (in view of Lemma 5.4), it follows that $d^{\subseteq}\left(B, \mathcal{Q A} \mathcal{P}_{d}\right)=0$ implies $B$ is an arithmetic progression, whence Lemma 5.8 completes the proof. So we can assume $d \subseteq(B, \mathcal{Q A \mathcal { P }} d)>0$.

By translating and considering $-A$ and $-B$ if necessary, we may w.l.o.g. assume $0, d \in A$, and that 0 is the first term of the minimal arithmetic progression with difference $d$ containing $A$. Since $\langle d\rangle=G$, let $B_{1}, \ldots, B_{c}$ be the $d$-components of $B$ cyclicly ordered according to the direction given by $d$. Let $B_{i}^{\prime}$ be the $d$-component of $\bar{B}$ located between $B_{i}$ and $B_{i+1}$, with indices taken modulo $c$. Hence $G$ is the disjoint union of the $B_{i}$ and $B_{i}^{\prime}$. Observe, since 0 and $d$ are the first two terms in $A$, that for each $i$ either at least $\min \left\{|A|,\left|B_{i}^{\prime}\right|\right\}$ of the holes contained in $B_{i}^{\prime}$ are elements of $A+B$, or else $\left|B_{i}\right|=1$ and at least $\max \left\{1, \min \left\{|A|-1,\left|B_{i}^{\prime}\right|-1\right\}\right\}$ 
of the holes contained in $B_{i}^{\prime}$ are elements of $A+B$. Hence, since $|B|+2(|A|-1)>|A|+|B|$, it follows that $\left|B_{i}^{\prime}\right|<|A|$ for all but at most one $i$ (say $c$ ).

If $|A|=3$, then $|A+B|=|B|+3$ implies $c \leq 3$. Hence if $\left|B_{i}^{\prime}\right|<|A|$ for all $i$, then $|A+B| \geq|\langle A\rangle|-c \geq|G|-3$. Thus $|A|=|\overline{A+B}|=3$, a contradiction. If $|A| \geq 4$, then in view of Lemma 5.9 it follows that $c \leq 2$, else the proof is complete. Hence if $\left|B_{i}^{\prime}\right|<|A|$ for all $i$, then $|A+B| \geq|\langle A\rangle|-c \geq|G|-2$, contradicting $d \subseteq(A+B, \mathcal{P}) \geq 3$. Thus, regardless of $|A|$, we can assume $\left|B_{c}^{\prime}\right| \geq|A|$. Consequently, the discussion of the previous paragraph implies

$$
|A+B| \geq|A|+|B|+d^{\subseteq}\left(B, \mathcal{Q} \mathcal{A} \mathcal{P}_{d}\right)-x+y
$$

where $x$ is the number of $B_{i}$ with $\left|B_{i}\right|=1$, and $y$ is the number of $B_{i}^{\prime}$ with $\left|B_{i}\right|=\left|B_{i}^{\prime}\right|=1$.

Suppose $|A|=3$. Hence $A=\{0, d, 3 d\}$, and as noted in the previous paragraph, $c \leq 3$. Since $|B| \geq 4$, it follows that $x \leq c-1 \leq 2$. On the other hand, $d \subseteq\left(B, \mathcal{Q} \mathcal{A} \mathcal{P}_{d}\right) \geq c-1$, with equality possible only if $\left|B_{i}^{\prime}\right|=1$ for $i \leq c-1$. Thus from (38) it follows that $|A|+|B|=|A+B| \geq|A|+|B|+d \subseteq\left(B, \mathcal{Q} \mathcal{A} \mathcal{P}_{d}\right)-x+y \geq|A|+|B|$, whence indeed $\left|B_{i}^{\prime}\right|=1$ for $i \leq c-1$ and $y=0$. Hence if $c=2$, then $d^{\subseteq}\left(B, \mathcal{Q} \mathcal{A} \mathcal{P}_{d}\right)=1$, whence letting $\alpha$ be the hole in $A$ and letting $\beta$ be the hole in $B$ yields (17) (recall that $\langle d\rangle=G$ ). If $c=1$, then $d^{\subseteq}\left(B, \mathcal{Q} \mathcal{A} \mathcal{P}_{d}\right)=0$, a contradiction to the conclusion of the first paragraph. Therefore assume $c=3$. Hence, since each $B_{i}^{\prime}$ contributes at least one to the sumset, and since $B_{3}^{\prime}$ contributes at least $|A|-1$ (in view of the discussion in the second paragraph of the proof), it follows that $|A+B| \geq|B|+|A|-1+(c-1)>|A|+|B|$, again a contradiction. So we may assume $|A| \geq 4$, and (as noted before) that $c \leq 2$.

Since $|B| \geq 3$ and since $c \leq 2$, it follows that at most one $B_{i}$ can have cardinality one. Hence from (38) it follows that $|A+B| \geq|A|+|B|+d^{\subseteq}\left(B, \mathcal{Q} \mathcal{A} \mathcal{P}_{d}\right)-1$, whence $d^{\subseteq}\left(B, \mathcal{Q} \mathcal{A} \mathcal{P}_{d}\right)=1$. Letting $\alpha$ be the hole in $A$, and letting $\beta$ be the hole in $B$ yields (17), and completes the proof.

We conclude the list of lemmas with a short proof of a special case of the Fainting Lemma from [16]. We remark that the idea for the proof of Lemma 5.11 could be used to prove a weaker form of the Fainting Lemma that does not require the assumption about the first isoperimetric number.

Lemma 5.11. Let $A$ and $B$ be finite, nonempty subsets of an abelian group $G$ with $0 \in A \cap B,|A|=3$, $|A+B|=|A|+|B|+m$, and $\langle A\rangle=G$. If $r_{A, B}(c) \geq 2$ for all $c \in A+B$, then $G$ is finite and $|B| \geq|G|-\left(\begin{array}{c}m+4 \\ 2\end{array}\right)$.

Proof. In view of Proposition 2.2, it follows that $r_{B+(i-1) A, A}(c) \geq 2$ for all $c \in A+i B$ and $i \geq 1$. Hence, since $|A|=3$, it follows that $N_{i}^{A^{*}}=N_{i}^{\leq A^{*}}=N_{i}$ for all $i \geq 1$, where $A^{*}=A \backslash 0$. Thus in view of Proposition 2.3 it follows that

$$
N_{i}-A^{*} \subseteq N_{i-1}
$$

for all $i \geq 2$. Note since $\langle A\rangle=G$, that either $B+l A=G$ for sufficiently large $l$, if $G$ is finite, or else $|B+l A|>|B+(l-1) A|$ for all $l$ (in view of Kneser's Theorem), if $G$ is infinite. Thus if we can show that $\left|N_{i}\right|<\left|N_{i-1}\right|$ for nonempty $N_{i-1}$ with $i \geq 2$, it will follow that $N_{i}=\emptyset$ for sufficiently large $i$, whence $G$ is finite, and that $|B|=|G|-\sum_{i>1}\left|N_{i}\right| \geq|G|-\sum_{i=0}^{m+2}(m+3-i)=|G|-\left(\begin{array}{c}m+4 \\ 2\end{array}\right)$, completing the proof. However, if $\left|N_{i}\right| \geq\left|N_{i-1}\right|>0$, then in view of (39) and Kneser's Theorem, it follows that $\left|N_{i}\right|=\left|N_{i-1}\right|$, that $N_{i}$ is periodic with maximal period (say) $H$, and that $A^{*}$ is a subset of an $H$-coset. Since $r_{B+(i-1) A, A}(c) \geq 2$ for all $c \in B+i A$, it follows that $B+i A=B+(i-1) A+A^{*}$. Thus, since $\left|\phi_{H}\left(A^{*}\right)\right|=1$, it follows that $\phi_{H}(B+(i-1) A)=\phi_{H}(B+i A)$, whence $N_{i}=(B+i A) \backslash(B+(i-1) A)$ cannot be $H$-periodic, a contradiction. Therefore $\left|N_{i}\right|<\left|N_{i-1}\right|$, completing the proof. 


\section{The Main Proof}

We are now ready to proceed with the proof of Theorem 4.1. However, before beginning, we sketch the main points to outline the strategy. We begin by handling the case $A+B$ periodic, and then show that we can restrict our attention to the case when neither $A$ nor $B$ is quasi-periodic. We then handle the cases when $d \subseteq(A+B, \mathcal{P})$ is small. The assumption that $d \subseteq(A+B, \mathcal{P})$ is small will allow us (in most instances) to show (17) fairly easily, since adding $H$-holes to $A$ or $B$ can only increase $A+B$ by at most $d^{\subseteq}\left(A+B, \mathcal{P}_{H}\right)$ elements. However, there will be one difficult instance (Claim 5) that will instead lead to the type (VIII) pair. Once we have established that $d^{\subseteq}(A+B, \mathcal{P}) \geq 3$, we can restrict our attention to generating subsets and begin to gain access to the lemmas we have just proved. To gain full access, we must handle the case when $|A| \leq 3$. The case $|A|=|B|=3$ is handled by brute force. We then restrict our attention to the case $G$ finite. For the case $|A|=3$ with $|B| \geq 4$, we use Lemma 5.11 to show the existence of a unique expression element $a+b$, and proceed by inductive arguments used on the pair $(A, B \backslash b)$. These will fail if $A \subseteq B$ and $|B|=4$, in which case an additional argument is used. With the cases $\min \{|A|,|B|\} \leq 3$ complete, the proof then continues, for $G$ finite, by induction (assuming the theorem true for $A^{\prime}$ and $B^{\prime}$ with $\min \left\{\left|A^{\prime}\right|,\left|B^{\prime}\right|\right\}<\min \{|A|,|B|\}$ or $\min \left\{\left|A^{\prime}\right|,\left|B^{\prime}\right|\right\}=\min \{|A|,|B|\}$ and $\left.\left|A^{\prime}\right|+\left|B^{\prime}\right|>|A|+|B|\right)$. We employ the previously mentioned Dyson $e$-transform as the method to obtain the pairs $A^{\prime}+B^{\prime}=C^{\prime}$. The arguments from the proof of Kneser's Theorem in [11] [38] will be extended to handle the case when $A(e)+B(e)$ is periodic. The cases $|B(e)| \geq 3$ are handled by applying the induction hypothesis to the pair $(A(e), B(e))$. This method fails when $|B(e)| \leq 2$, since in these cases the unpaired structural information gained for a single set is insufficient to directly transfer back to the original pair. In the case $|B(e)|=1$, we instead use the method developed in Section 3. In the case $|B(e)|=2$, then via Proposition 2.4 we will obtain $c_{d}(A) \leq 2$ for some nonzero $d$, whence we instead consider $A+\{0, d\}+B$, as discussed before Lemma 5.9. We will encounter problems if $|\overline{A+B}|$ is small. The remaining cases will then be shown to follow from the case $|A|=|B|=4$ with $c_{d}(A)=c_{d}(B)=c_{d}(A+B)=2$. The proof with $G$ finite concludes by completing this last remaining case directly. The case when $G$ is infinite is then derived from the finite case by the use of an appropriate Freiman isomorphism of $(A, B)$ (which is an injective map $\varphi: A \cup B \rightarrow G^{\prime}$, with $G^{\prime}$ an abelian group, such that $\varphi\left(a_{1}\right)+\varphi\left(b_{1}\right)=\varphi\left(a_{2}\right)+\varphi\left(b_{2}\right)$ holds, where $a_{i} \in A$ and $b_{i} \in B$, if and only if $\left.a_{1}+b_{1}=a_{2}+b_{2}\right)$.

Proof. We may assume w.l.o.g. that

$$
0 \in A \cap B .
$$

If either $A$ or $B$ is extendible, then (17) immediately follows. Therefore we can assume otherwise, whence Proposition 2.4 implies

$$
-A+\overline{A+B}=\bar{B} \text { and }-B+\overline{A+B}=\bar{A} .
$$

Claim 1: $d \subseteq(A+B, \mathcal{P}) \geq 1$, else the proof is complete.

Suppose instead that $A+B$ is periodic with $H=H(A+B)$. If $A$ and $B$ are not both $H$-periodic, then w.l.o.g. there exists $\alpha \in \bar{A}$ such that $\phi_{H}(A)=\phi_{H}(A \cup\{\alpha\})$. Hence, since $A+B$ is $H$-periodic, it follows that $(A \cup\{\alpha\})+B=A+B$, contradicting that $A$ is non-extendible. Therefore we may assume that $A$ and $B$ are both $H$-periodic. From Kneser's Theorem it follows that

$$
\left|\phi_{H}(A+B)\right| \geq\left|\phi_{H}(A)\right|+\left|\phi_{H}(B)\right|-1 .
$$

If equality holds in the above inequality, then since $A$ and $B$ are both $H$-periodic, it follows that

$$
|A+B|=|H|\left|\phi_{H}(A+B)\right|=|H+A|+|H+B|-|H|=|A|+|B|-|H|<|A|+|B|,
$$


a contradiction. If $\left|\phi_{H}(A+B)\right|=\left|\phi_{H}(A)\right|+\left|\phi_{H}(B)\right|$, then the proof is complete. Otherwise

$$
|A+B|=|H|\left|\phi_{H}(A+B)\right| \geq|H+A|+|H+B|+|H|=|A|+|B|+|H|>|A|+|B|,
$$

a contradiction once more. So we may assume that $A+B$ is aperiodic.

Claim 2: $\quad d \subseteq(A+B, \mathcal{P}) \geq 2$.

Suppose instead, for some $\gamma \in \overline{A+B}$, that $(A+B) \cup\{\gamma\}$ is periodic with maximal period (say) $H$. Note $\phi_{H}(\gamma) \in \phi_{H}(A+B)$. Hence choosing $\alpha \in(\gamma-B) \cap(H+A)$ and $\beta \in(\gamma-A) \cap(H+B)$, it follows that $(A \cup\{\alpha\})+(B \cup\{\beta\})=(A+B) \cup\{\gamma\}$, whence (17) holds. So we may assume $d \subseteq(A+B, \mathcal{P}) \geq 2$.

Claim 3: $|A|,|B| \geq 3$.

If $|A|=1$, then $|A+B|=|A|+|B|$ cannot hold, and if $|A|=2$, then the theorem holds with type (V) and group $G$. So we can assume $|A|,|B| \geq 3$.

Claim 4: It suffices to prove Theorem 4.1 in the case both $A$ and $B$ are non-quasi-periodic sets with $\langle A\rangle=\langle B\rangle=G$.

In view of Claim 1 and previous assumptions, the hypotheses of Lemma 5.3 hold for $A$ and $B$. However, it is readily checked that if $A=A_{1} \cup A_{0}$ and $B=B_{1} \cup B_{0}$ are quasi-periodic decompositions satisfying the conclusion of Lemma 5.3, and if Theorem 4.1 holds for the pair $\left(A_{0}, B_{0}\right)$, then Theorem 4.1 holds for the pair $(A, B)$. Thus it follows in view of Lemma 5.3 (by considering reduced quasi-periodic decompositions) that it suffices to prove the theorem when neither $A$ nor $B$ is quasi-periodic, as the following more detailed argument shows.

Let $A=A_{1} \cup A_{0}$ be a reduced quasi-periodic decomposition with $H=H\left(A_{1}\right)$ and apply Lemma 5.3 using $A=A_{1} \cup A_{0}$, yielding the corresponding quasi-periodic decomposition $B_{1} \cup B_{0}$ for $B$ with quasi-period $H$. If $B_{1} \cup B_{0}$ is not reduced, then let $B=B_{1}^{\prime} \cup B_{0}^{\prime}$ be a reduced quasi-periodic decomposition for $B$ with quasi-period $H^{\prime}$. As mentioned after defining quasi-periodic in Section 2, we may assume $B_{0}^{\prime} \subseteq B_{0}$ and $H^{\prime} \leq H$. Now apply Lemma 5.3 using $B=B_{1}^{\prime} \cup B_{0}^{\prime}$ instead (take $B_{i}^{\prime}=B_{i}$ if $B_{1} \cup B_{0}$ was already reduced), and let $A_{1}^{\prime} \cup A_{0}^{\prime}$ be the resulting quasi-periodic decomposition for $A$. Since $H^{\prime} \leq H$, and since $A=A_{1} \cup A_{0}$ was a reduced quasi-periodic decomposition with quasi-period $H$, we conclude that $A_{1}^{\prime}=A_{1}$ and $A_{0}^{\prime}=A_{0}$. Thus we see that there exists a pair of reduced quasi-periodic decompositions $A=A_{1}^{\prime} \cup A_{0}^{\prime}$ and $B=B_{1}^{\prime} \cup B_{0}^{\prime}$ that satisfy the conclusion of Lemma 5.3. By the definition of reduced, both $A_{0}^{\prime}$ and $B_{0}^{\prime}$ are non-quasi-periodic sets. As mentioned in the previous paragraph, if Theorem 4.1 holds for the pair of non-quasi-periodic sets $\left(A_{0}^{\prime}, B_{0}^{\prime}\right)$, then it holds for the original pair $(A, B)$. As a result, we conclude that if we know Theorem 4.1 holds for an arbitrary pair of non-quasi-periodic sets, then it will hold in particular for the pair $\left(A_{0}^{\prime}, B_{0}^{\prime}\right)$, and thus also for $(A, B)$, and so it suffices to prove Theorem 4.1 in the case both $A$ and $B$ are non-quasi-periodic, which we henceforth assume. Thus it follows in view of Lemma 5.2 - which we can apply to $(A, B)$ by Claims 1 and 3 -that w.l.o.g. we may also assume $\langle A\rangle=\langle B\rangle=G$ (recall $0 \in A \cap B$ ).

Claim 5: $\quad d \subseteq(A+B, \mathcal{P}) \geq 3$.

Suppose instead (in view of Claim 2), for some distinct $\gamma_{1}, \gamma_{2} \in \overline{A+B}$, that $(A+B) \cup\left\{\gamma_{1}, \gamma_{2}\right\}$ is periodic with maximal period (say) $H$. Note that $\phi_{H}\left(\gamma_{i}\right) \in \phi_{H}(A+B)$, for $i=1,2$, since otherwise $A+B$ is periodic, contradicting Claim 1. Hence choosing $\alpha_{1} \in\left(\gamma_{1}-B\right) \cap(H+A)$ and $\beta_{1} \in\left(\gamma_{2}-A\right) \cap(H+B)$, it follows that $\left(A \cup\left\{\alpha_{1}\right\}\right)+\left(B \cup\left\{\beta_{1}\right\}\right)=(A+B) \cup\left\{\gamma_{1}, \gamma_{2}\right\}$. Since $d \subseteq(A+B, \mathcal{P}) \geq 2$ (Claim 2), it follows that either $A$ or $B$, w.l.o.g. $A$, contains at least two $H$-holes. Thus we can find $\alpha_{2} \in(H+A) \cap \overline{A \cup\{\alpha\}}$ such that $\left(A \cup\left\{\alpha_{1}, \alpha_{2}\right\}\right)+\left(B \cup\left\{\beta_{1}\right\}\right)=(A+B) \cup\left\{\gamma_{1}, \gamma_{2}\right\}$ is maximally $H$-periodic. Hence from Kneser's Theorem it follows that there are $\rho=|H|-1+3=|H|+2$ holes contained among the sets $A$ and $B$, and that

$$
\left|\phi_{H}(A+B)\right|=\left|\phi_{H}(A)\right|+\left|\phi_{H}(B)\right|-1 \text {. }
$$


Let $\left(\phi_{H}\left(a_{1}\right), \phi_{H}\left(b_{1}\right)\right),\left(\phi_{H}\left(a_{2}\right), \phi_{H}\left(b_{2}\right)\right), \ldots,\left(\phi_{H}\left(a_{l}\right), \phi_{H}\left(b_{l}\right)\right) \in \phi_{H}(A) \times \phi_{H}(B)$, with $a_{i} \in A$ and $b_{i} \in B$, be those pairs from $\phi_{H}(A) \times \phi_{H}(B)$ such that $\phi_{H}\left(a_{i}+b_{i}\right) \in\left\{\phi_{H}\left(\gamma_{1}\right), \phi_{H}\left(\gamma_{2}\right)\right\}$. Since $\gamma_{i} \notin A+B$, it follows in view of Proposition 2.1 that

$$
\left|A_{a_{i}}\right|+\left|B_{b_{i}}\right| \leq|H|
$$

for all $i$.

Suppose $\left|\left\{\phi_{H}\left(a_{i}\right)\right\}_{i=1}^{l}\right|=1$. Hence in view of the non-extendibility of $A$, it follows that $a+H \subseteq A$ for all $a \in A \backslash A_{a_{1}}$. Thus, since $A$ is non-quasi-periodic (Claim 4), it follows that $A=A_{a_{1}}$, whence $\langle A\rangle=G$ (Claim 4) implies $H=G$. Hence, since there are exactly $|G|-|B|$ elements $\alpha \in G$ such that $\gamma_{1} \notin \alpha+B$, and since $|A|+|B|=|A+B|=|G|-2$, it follows that there exists such an $\alpha \in \bar{A}$. Likewise, since there are exactly $|G|-|A|-1$ elements $\beta \in G$ such that $\gamma_{1} \notin \beta+(A \cup\{\alpha\})$, and since $|A|+|B|=|G|-2$, it follows that there exists such a $\beta \in \bar{B}$. Hence $(A \cup\{\alpha\})+(B \cup\{\beta\}) \subseteq G \backslash \gamma_{1}$, whence the non-extendibility of $(A, B)$ implies equality, yielding (17). So we can assume $\left|\left\{\phi_{H}\left(a_{i}\right)\right\}_{i=1}^{l}\right| \geq 2$. By the same argument, it also follows that $\left|\left\{\phi_{H}\left(b_{i}\right)\right\}_{i=1}^{l}\right| \geq 2$.

Hence in view of (41), it follows that $\rho \geq 2|H|$, with equality possible only if $\left|\left\{\phi_{H}\left(a_{i}\right)\right\}_{i=1}^{l}\right|=2$ and $\left|\left\{\phi_{H}\left(b_{i}\right)\right\}_{i=1}^{l}\right|=2$. Thus in view of $\rho=|H|+2$, it follows that $|H|=2$, that $\left|\left\{\phi_{H}\left(a_{i}\right)\right\}_{i=1}^{l}\right|=2$ and that $\left|\left\{\phi_{H}\left(b_{i}\right)\right\}_{i=1}^{l}\right|=2$, implying $\phi_{H}\left(\gamma_{1}\right) \neq \phi_{H}\left(\gamma_{2}\right)$ (else $A+B$ is periodic, contradicting Claim 1). There are three cases for $l$.

Suppose $l=2$. Hence w.l.o.g. $\phi_{H}\left(A_{a_{1}}\right)+\phi_{H}\left(B_{b_{1}}\right)=\phi_{H}\left(\gamma_{1}\right)$ and $\phi_{H}\left(A_{a_{2}}\right)+\phi_{H}\left(B_{b_{2}}\right)=\phi_{H}\left(\gamma_{2}\right)$ are both unique modulo $H$ expression elements, whence letting $\alpha$ be the other element from the $H$-coset $a_{1}+H$, and letting $\beta$ be the other element from the $H$-coset $b_{1}+H$, it follows that $(A \cup\{\alpha\})+(B \cup\{\beta\})=(A+B) \cup\left\{\gamma_{1}\right\}$, yielding (17). So we can assume $l>2$

Suppose $l=3$. Hence some $\phi_{H}\left(a_{i}\right)$, say $\phi_{H}\left(a_{j_{1}}\right)$, is contained in only one pair $\left(\phi_{H}\left(a_{i}\right), \phi_{H}\left(b_{i}\right)\right)$. Likewise some $\phi_{H}\left(b_{i}\right)$, say $\phi_{H}\left(b_{j_{2}}\right)$, is also only contained in one pair $\left(\phi_{H}\left(a_{i}\right), \phi_{H}\left(b_{i}\right)\right)$. Hence, since $l=3$, it follows that $b_{j_{1}} \neq b_{j_{2}}$ and $a_{j_{1}} \neq a_{j_{2}}$. Thus neither $\phi_{H}\left(a_{j_{2}}+b_{j_{2}}\right)$ nor $\phi_{H}\left(a_{j_{1}}+b_{j_{1}}\right)$ can equal $\phi_{H}\left(a_{j_{2}}+b_{j_{1}}\right) \in$ $\left\{\phi_{H}\left(\gamma_{1}\right), \phi_{H}\left(\gamma_{2}\right)\right\}$, whence $\phi_{H}\left(a_{j_{1}}+b_{j_{1}}\right)=\phi_{H}\left(a_{j_{2}}+b_{j_{2}}\right)$, and w.l.o.g. assume $\phi_{H}\left(a_{j_{1}}+b_{j_{1}}\right)=\phi_{H}\left(\gamma_{1}\right)$. Hence, letting $\alpha \in\left(\gamma_{1}-B\right) \cap\left(A_{a_{j_{1}}}+H\right)$ and letting $\beta \in\left(\gamma_{1}-A\right) \cap\left(B_{b_{j_{2}}}+H\right)$, it follows that $(A \cup\{\alpha\})+(B \cup\{\beta\})=$ $(A+B) \cup\left\{\gamma_{1}\right\}$, whence (17) holds. So we can assume $l=4$.

Let $A+B=C$. Hence, since each $C_{\gamma_{i}}$ is a partially filled $H$-coset with $|H|=2$, it follows in view of $l=4$ that

$$
C_{\gamma_{1}} \cup C_{\gamma_{2}}=\left(A_{a_{1}} \cup A_{a_{2}}\right)+\left(B_{b_{1}} \cup B_{b_{2}}\right)=b_{1}+\left(A_{a_{1}} \cup A_{a_{2}}\right)=a_{1}+\left(B_{b_{1}} \cup B_{b_{2}}\right),
$$

implying from Kneser's Theorem that $\left(A_{a_{1}} \cup A_{a_{2}}\right)+\left(B_{b_{1}} \cup B_{b_{2}}\right)=b_{1}+\left(A_{a_{1}} \cup A_{a_{2}}\right)=a_{1}+\left(B_{b_{1}} \cup B_{b_{2}}\right)$ is periodic with maximal period (say) $H^{\prime}$. Since $\left|B_{b_{1}} \cup B_{b_{2}}\right|=2$, it follows that $\left|H^{\prime}\right|=2$. Let $K=H \times H^{\prime} \cong \mathbb{Z} / 2 \mathbb{Z} \times \mathbb{Z} / 2 \mathbb{Z}$. Since $C_{\gamma_{1}} \cup C_{\gamma_{2}}$ is an $H^{\prime}$-coset, it follows that it does not contain an $H$-coset. Hence $\phi_{K}\left(\gamma_{1}\right)=\phi_{K}\left(a_{1}\right)+\phi_{K}\left(b_{1}\right)$ must be a unique expression element in $\phi_{K}(A)+\phi_{K}(B)$.

In view of the maximality of $H$, it follows that $\phi_{H}(A+B)$ is aperiodic. Hence, in view of (40), it follows that we can apply KST to the pair $\left(\phi_{H}(A), \phi_{H}(B)\right)$. Let $\phi_{H}\left(A_{1}^{\prime}\right) \cup \phi_{H}\left(A_{0}^{\prime}\right)$ and $\phi_{H}\left(B_{1}^{\prime}\right) \cup \phi_{H}\left(B_{0}^{\prime}\right)$ be the Kemperman decompositions with quasi-period $L / H$, where $A=A_{1}^{\prime} \cup A_{0}^{\prime}$ and $B=B_{1}^{\prime} \cup B_{0}^{\prime}$. Since $\phi_{K}\left(\left\{a_{1}, a_{2}\right\}+\left\{b_{1}, b_{2}\right\}\right)$ is a unique expression element, it follows that both elements in $\phi_{H}\left(\left\{a_{1}, a_{2}\right\}+\left\{b_{1}, b_{2}\right\}\right)$ have exactly two representations in $\phi_{H}(A)+\phi_{H}(B)$ given by

$$
\phi_{H}\left(a_{1}\right)+\phi_{H}\left(b_{2}\right)=\phi_{H}\left(a_{2}\right)+\phi_{H}\left(b_{1}\right) \text { and } \phi_{H}\left(a_{1}\right)+\phi_{H}\left(b_{1}\right)=\phi_{H}\left(a_{2}\right)+\phi_{H}\left(b_{2}\right) .
$$

Since $\phi_{H}(A+B)$ is aperiodic, it follows that we cannot have type (III). Suppose we have type (II). If say $a_{1} \in A_{1}^{\prime}$, then (since type (II) implies $\left|A_{x}\right|,\left|B_{y}\right| \geq 2$ for all $x \in A$ and $y \in B$ ), it follows in view of (42) that $\phi_{L}\left(a_{1}\right)=\phi_{L}\left(a_{2}\right)$ and that $\phi_{L}\left(b_{1}\right)=\phi_{L}\left(b_{2}\right)$. Furthermore, if $b_{i} \in B_{1}^{\prime}$, then $|L / H|=2$, and if 
$b_{i} \in B_{0}^{\prime}$, then $\left|\phi_{H}\left(B_{0}^{\prime}\right)\right|=2$. However, since $\phi_{H}\left(B_{0}^{\prime}\right)$ is an arithmetic progression, and since $\left\{\phi_{H}\left(b_{1}\right), \phi_{H}\left(b_{2}\right)\right\}$ is periodic with period $K / H$, it follows that $|L / H|=2$ in this latter case as well. However, $|L / H|=2$ is impossible for type (II) (since the order of the difference of the arithmetic progression given by KST is at least $\left.\left|\phi_{H}\left(A_{0}^{\prime}\right)\right|+\left|\phi_{H}\left(A_{1}^{\prime}\right)\right|-1 \geq 3\right)$. Therefore, we can assume $a_{1}, a_{2} \in A_{0}^{\prime}$ and $b_{1}, b_{2} \in B_{0}^{\prime}$. Hence, since $\phi_{H}\left(\left\{a_{1}, a_{2}\right\}\right)$ is an $K / H$-coset with $|K / H|=2$, and since $\phi_{H}\left(A_{0}^{\prime}\right)$ is an arithmetic progression whose difference generates the cyclic group $\left\langle\phi_{H}\left(A_{0}^{\prime}-a_{1}\right)\right\rangle=\left\langle\phi_{H}\left(B_{0}^{\prime}-b_{1}\right)\right\rangle \leq L / H$, it follows that $\left|\phi_{H}\left(A_{0}^{\prime}\right)\right|>$ $\frac{1}{2}\left|\left\langle\phi_{H}\left(A_{0}^{\prime}-a_{1}\right)\right\rangle\right|$. Likewise $\left|\phi_{H}\left(B_{0}^{\prime}\right)\right|>\frac{1}{2}\left|\left\langle\phi_{H}\left(B_{0}^{\prime}-b_{1}\right)\right\rangle\right|=\frac{1}{2}\left|\left\langle\phi_{H}\left(A_{0}^{\prime}-a_{1}\right)\right\rangle\right|$, whence Proposition 2.1 implies that $\phi_{H}(A+B)$ is $\left\langle\phi_{H}\left(A_{0}^{\prime}-a_{1}\right)\right\rangle$-periodic, a contradiction. So type (II) cannot occur.

Suppose we have type (I) with w.l.o.g. $\left|\phi_{H}\left(A_{0}^{\prime}\right)\right|=1$. Hence some $a_{i}$, say $a_{2}$, is contained in $A_{1}^{\prime}$. Thus, if some $b_{i}$ is contained in $B_{0}^{\prime}$, then it follows in view of (42) that either $\left|\phi_{H}\left(B_{0}^{\prime}\right)\right|=2, \phi_{L}\left(a_{1}\right)=\phi_{L}\left(a_{2}\right)$ and $b_{1}, b_{2} \in B_{0}^{\prime}$ (if $\left|\phi_{H}\left(B_{0}^{\prime}\right)\right| \geq 2$ ), or else w.l.o.g. $\phi_{H}\left(B_{0}^{\prime}\right)=\left\{\phi_{H}\left(b_{2}\right)\right\}$ and $\phi_{H}\left(A_{0}^{\prime}\right)=\left\{\phi_{H}\left(a_{1}\right)\right\}\left(\right.$ if $\left.\left|\phi_{H}\left(B_{0}^{\prime}\right)\right|=1\right)$. In the former case $\phi_{H}\left(B_{0}^{\prime}\right)$, and thus $\phi_{H}(B)$, is $K / H$-periodic, contradicting that $\phi_{H}(A+B)$ is aperiodic. In the later case, $\phi_{H}\left(a_{1}\right)+\phi_{H}\left(b_{2}\right)=\phi_{H}\left(a_{2}\right)+\phi_{H}\left(b_{1}\right)$ contradicts that $\phi_{H}\left(A_{0}^{\prime}\right)+\phi_{H}\left(B_{0}^{\prime}\right)=\phi_{H}\left(a_{1}\right)+\phi_{H}\left(b_{2}\right)$ is a unique expression element. Therefore we can assume $b_{1}, b_{2} \in B_{1}^{\prime}$. Thus, since $a_{2} \in A_{1}^{\prime}$, it follows in view of (42) that $\phi_{L}\left(a_{1}\right)=\phi_{L}\left(a_{2}\right), \phi_{L}\left(b_{1}\right)=\phi_{L}\left(b_{2}\right)$, and $|L / H|=2$. Since $\phi_{L}\left(a_{1}\right)=\phi_{L}\left(a_{2}\right)$, it follows that $K / H \leq L / H$. Hence $|L / H|=2$ implies that $L=K$. Since $|L / H|=2$, and since $\phi_{H}(A+B)$ is aperiodic, it follows that $\left|\phi_{H}\left(A_{0}^{\prime}\right)\right|=\left|\phi_{H}\left(B_{0}^{\prime}\right)\right|=1$. Since $L=K$, and since $\phi_{K}\left(a_{1}\right)+\phi_{K}\left(b_{1}\right)$ is a unique expression element, it follows that $\phi_{L}\left(a_{1}\right)+\phi_{L}\left(b_{1}\right)$ is a unique expression element in addition to the unique expression element $\phi_{L}\left(A_{0}^{\prime}\right)+\phi_{L}\left(B_{0}^{\prime}\right)$. Hence, since $a_{1} \notin A_{0}^{\prime}$ and since $b_{1} \notin B_{0}^{\prime}$, then applying KST modulo $L=K$, it follows that we must have type (II) with (by appropriate choice of sign) both $\phi_{L}\left(a_{i}\right)$ and $\phi_{L}\left(b_{i}\right)$ the first term in their respective arithmetic progression, and both $\phi_{L}\left(A_{0}^{\prime}\right)$ and $\phi_{L}\left(B_{0}^{\prime}\right)$ the last term in their respective arithmetic progression. Thus the theorem holds with type (VIII).

Finally, suppose we have type (IV). Hence $\left|\phi_{H}\left(A_{0}^{\prime}\right)\right|,\left|\phi_{H}\left(B_{0}^{\prime}\right)\right| \geq 3$, and $\phi_{H}\left(A_{0}^{\prime}\right)$ is aperiodic (all consequences for a type (IV) pair, as remarked in Section 2). If we do not have $a_{1}, a_{2} \in A_{0}^{\prime}$ and $b_{1}, b_{2} \in B_{0}^{\prime}$, say instead $b_{2} \in B_{1}^{\prime}$, then $\left|\phi_{H}\left(A_{0}^{\prime}\right)\right| \geq 3$ implies that every element of the form $\phi_{H}(a)+\phi_{H}\left(b_{2}\right) \in \phi_{H}(A+B)$, where $\phi_{H}(a) \in \phi_{H}(A)$, has at least three representations in $\phi_{H}(A)+\phi_{H}(B)$, which contradicts (42). Therefore we may assume $a_{1}, a_{2} \in A_{0}^{\prime}$ and $b_{1}, b_{2} \in B_{0}^{\prime}$. Hence $K<L$. Thus, since $\phi_{K}\left(a_{i}\right)+\phi_{K}\left(b_{i}\right)$ is a unique expression element, it follows in view of Proposition 2.1 that

$$
\left|\phi_{K}\left(A_{0}^{\prime}+B_{0}^{\prime}\right)\right| \geq\left|\phi_{K}\left(A_{0}^{\prime}\right)\right|+\left|\phi_{K}\left(B_{0}^{\prime}\right)\right|-1
$$

In view of the description of type (IV), it follows that $\left|\phi_{K}\left(A_{0}^{\prime}\right)\right|=l_{1}+l_{p}$ and $\left|\phi_{K}\left(B_{0}^{\prime}\right)\right|=l_{2}+l_{p}$, where $l_{p}$ is the number of partially filled $K / H$-cosets in $\phi_{H}\left(A_{0}^{\prime}\right)$, which is also equal to the number of partially filled $K / H$-cosets in $\phi_{H}\left(B_{0}^{\prime}\right)$, where $l_{1}$ is the number of $\phi_{H}(x) \in \phi_{H}\left(A_{0}^{\prime}\right)$ with $\phi_{H}(x)+K / H \subseteq \phi_{H}\left(A_{0}^{\prime}\right)$, and where $l_{1}+l_{2}+l_{p}=|L / K|$. Thus $\left|\phi_{K}\left(A_{0}^{\prime}\right)\right|+\left|\phi_{K}\left(B_{0}^{\prime}\right)\right|-1=|L / K|+l_{p}-1$. Hence, since $\left|\phi_{K}\left(A_{0}^{\prime}+B_{0}^{\prime}\right)\right| \leq|L / K|$ holds trivially, and since $\phi_{H}\left(A_{0}^{\prime}\right)$ is aperiodic, it follows in view of (43) that $l_{p}=1$. Let $\phi_{H}(x) \in \phi_{H}\left(A_{0}^{\prime}\right)$ and $\phi_{H}(y) \in \phi_{H}\left(B_{0}^{\prime}\right)$ be the elements that correspond to the unique partially filled $K / H$-coset in $\phi_{H}\left(A_{0}^{\prime}\right)$ and $\phi_{H}\left(B_{0}^{\prime}\right)$, respectively. Hence, since $\phi_{H}(A+B)$ is aperiodic, and since $l_{p}=1$, it follows that $\phi_{K}(x)+\phi_{K}(y)$ is a unique expression element in $\phi_{K}\left(A_{0}^{\prime}\right)+\phi_{K}\left(B_{0}^{\prime}\right)$. However, since $|K / H|=2$, it follows that there is only one element from the coset $x+K / H$ contained in $\phi_{H}\left(A_{0}^{\prime}\right)$, and likewise for the coset $y+K / H$ in $\phi_{H}\left(B_{0}^{\prime}\right)$, whence $\phi_{H}(x)+\phi_{H}(y)$ is a unique expression element, contradicting that there are no unique expression elements in a type (IV) pair, and completing the case when $d \subseteq(A+B, \mathcal{P}) \leq 2$. So we can assume $d \subseteq(A+B, \mathcal{P}) \geq 3$.

Claim 6: $\max \{|A|,|B|\} \geq 4$, say $|B| \geq 4$.

Suppose instead that $|A|=|B|=3$ (in view of Claim 3). If $(A-A) \cap(B-B)=\{0\}$, then $|A+B|=$ $|A||B|=9>6=|A|+|B|$, a contradiction. Thus w.l.o.g. $A=\left\{0, d, a_{1}\right\}$ and $B=\left\{0, d, a_{2}\right\}$. In view of 
Lemma 5.8 - which we can apply to $(A, B)$ in view of Claims 1 and 3 -it follows that we can assume neither $A$ nor $B$ is an arithmetic progression, else the proof is complete. Since $A$ is not quasi-periodic (Claim 4) and $|A|=3$, it follows that no two elements from $A$ can form a coset of an order two subgroup. Likewise for $B$.

Note

$$
A+B=\left\{0, d, 2 d, a_{1}, a_{1}+d, a_{2}, a_{2}+d, a_{1}+a_{2}\right\}
$$

If $a_{1}=a_{2}$, then the theorem follows with type (VI). If $|\{0, d, 2 d\}| \leq 2$, then $\{0, d\}$ is a subgroup of order 2 , which we have noted is not the case. Therefore $|\{0, d, 2 d\}|=3$ and $2 d \neq 0$. Since neither $A$ nor $B$ is an arithmetic progression, it follows that $a_{1}, a_{2} \notin\{2 d,-d\}$. Hence, since $a_{1}+d=a_{2}$ and $a_{2}+d=a_{1}$ together contradict that $2 d \neq 0$, it follows that

$$
\left|\left\{0, d, 2 d, a_{1}, a_{1}+d, a_{2}, a_{2}+d\right\}\right| \geq|\{0, d, 2 d\}|+\left|\left\{a_{1}, a_{1}+d, a_{2}, a_{2}+d\right\}\right| \geq 6,
$$

with equality possible only if w.l.o.g. $a_{2}=a_{1}+d$. Thus in view of (44) and $|A+B|=6$, it follows that

$$
a_{1}+a_{2}=2 a_{1}+d \in A+B=\left\{0, d, 2 d, a_{1}, a_{1}+d, a_{1}+2 d\right\},
$$

implying that one of the following hold: $2 a_{1}+d=0,2 a_{1}=0,2 a_{1}=d, a_{1}=-d, a_{1}=0$, or $a_{1}=d$. The last three equalities are contradictions. If $2 a_{1}=0$, then $\left\{0, a_{1}\right\}$ is a coset of an order two subgroup, if $2 a_{1}=d$, then $A=\left\{0, d, a_{1}\right\}=\left\{0,2 a_{1}, a_{1}\right\}$ is an arithmetic progression, and if $-2 a_{1}=d$, then $B=\left\{0, d, a_{1}+d\right\}=$ $\left\{0,-2 a_{1},-a_{1}\right\}$ is an arithmetic progression, all contradictions as well. So we can assume w.l.o.g. $|B| \geq 4$.

\section{CASE I: $G$ is Finite.}

At this point we assume $G$ is finite, and will handle the case $G$ infinite afterwards by a short, separate argument. Consequently, in view of Lemma 5.4 - which we can apply to $(A, B)$ in view of Claims 3,4 and 5 (note Claim 5 implies $|\overline{A+B}| \geq 3$ ) -it follows that

$$
d^{\subseteq}(A+B, \mathcal{Q P}) \geq 1 \text { and } d^{\subseteq}(\overline{A+B}, \mathcal{Q P}) \geq 1
$$

and that

$$
\langle-\gamma+\overline{A+B}\rangle=G,
$$

where $\gamma \in \overline{A+B}$. In view of Lemma 5.7 - which we can apply to $(A, B)$ in view of Claims $3,4,5$ and 6 - it follows that we can assume

$$
d^{\subseteq}(A, \mathcal{Q P}), d^{\subseteq}(B, \mathcal{Q P}) \geq 2,
$$

else the proof is complete. In view of Lemma 5.6 - which we can apply to $(A, B)$ in view of Claims 3,4 and 5 - it follows that we can assume

$$
d^{\subseteq}(\bar{B}, \mathcal{P}), d^{\subseteq}(\bar{A}, \mathcal{P}) \geq 3
$$

else the proof is complete, whence in view of Lemma 5.7 - which we can apply to $(-B,-\gamma+\overline{A+B})$, where $\gamma \in \overline{A+B}$, in view of Proposition 2.4, Claims 3, 5 and 6, (45), (46) and (48) - it follows that we can assume

$$
d^{\subseteq}(\overline{A+B}, \mathcal{Q P}) \geq 2,
$$

else $d \subseteq(-B, \mathcal{Q P})=d \subseteq(B, \mathcal{Q P})=1$, a contradiction to $(47)$.

Claim 8: $\left|N_{1}^{b}(A, B)\right| \leq 1$ and $\left|N_{1}^{a}(B, A)\right| \leq 1$, for all $b \in B$ and $a \in A$.

Suppose $\left|N_{1}^{b}(A, B)\right| \geq 2$ for some $b \in B$. If $A+(B \backslash b)$ is periodic with maximal period (say) $H$, then the non-extendibility of $B$ implies that $B \backslash b$ is $H$-periodic, whence $B$ is quasi-periodic, a contradiction to (47). Hence $A+(B \backslash b)$ is aperiodic, whence Kneser's Theorem implies $\left|N_{1}^{b}(A, B)\right|=2$. Thus we can apply $\mathrm{KST}$ to the pair $(A, B \backslash b)$. Since $\langle A\rangle=G$ and since $A$ is non-quasi-periodic (Claim 4 ), it follows that the 
quasi-period from KST must be $G$. Hence, since $|A|,|B \backslash b|>1$ (Claim 3), and since $|A+(B \backslash b)| \leq|G|-2$ (in view of $\left|N_{1}^{b}(A, B)\right| \geq 2$ ), it follows from KST that both $A$ and $B \backslash b$ are arithmetic progressions, whence Lemma 5.8- which we can apply to $(A, B)$ in view of Claims 1 and 3 -completes the proof. So we can assume $\left|N_{1}^{b}(A, B)\right| \leq 1$ for all $b \in B$. Likewise, $\left|N_{1}^{a}(B, A)\right| \leq 1$ for all $a \in A$.

If $|A|=|\overline{A+B}|=3$, then in view of Proposition 2.4 and the completed case $|A|=|B|=3$ (done in Claim 6), it follows that the theorem holds with type (VII). Therefore we can assume (in view of Claim 6) that at most one of $|A|,|B|$ and $|\overline{A+B}|$ is equal to three.

If $d \subseteq\left(A, \mathcal{Q} \mathcal{A} \mathcal{P}_{d}\right) \leq 1$, then $d \subseteq(A, \mathcal{Q P}) \geq 2$ (eq. (47)) implies that $d \subseteq(A, \mathcal{A P}) \leq 1$. Likewise for $B$. Thus in view of Lemmas 5.8 and 5.10 - which we can apply to $(A, B)$ in view of Claims 3,4 and 5 and the previous paragraph - it follows that

$$
d^{\subseteq}\left(A, \mathcal{Q} \mathcal{A} \mathcal{P}_{d}\right), d^{\subseteq}\left(B, \mathcal{Q} \mathcal{A} \mathcal{P}_{d}\right) \geq 2
$$

for all nonzero $d \in G$, else the proof is complete. Likewise, if $d \subseteq\left(\overline{A+B}, \mathcal{Q} \mathcal{A} \mathcal{P}_{d}\right) \leq 1$, then $d \subseteq(\overline{A+B}, \mathcal{Q P}) \geq 2$ (eq. (49)) implies that $d \subseteq(\overline{A+B}, \mathcal{A P}) \leq 1$, whence in view of Lemmas 5.8 and 5.10 - which we can apply to $(-B,-\gamma+\overline{A+B})$, where $\gamma \in \overline{A+B}$, in view of Proposition 2.4, Claims 3 and 5, (45), (46), (48) and the previous paragraph - it follows that either $d \subseteq\left(B, \mathcal{Q} \mathcal{A} \mathcal{P}_{d^{\prime}}\right) \leq 1$ for some nonzero $d^{\prime} \in G$, or else $d^{\subseteq}(B, \mathcal{Q P}) \leq 1$, both contradictions (to (50) or (47)). Therefore we can assume $d^{\subseteq}\left(\overline{A+B}, \mathcal{Q} \mathcal{A} \mathcal{P}_{d}\right) \geq 2$ for all nonzero $d \in G$ as well. Consequently,

$$
d^{\subseteq}(C, \mathcal{A P}) \geq 2
$$

for $C \in\{A, B, \overline{A+B}\}$.

Claim 9: w.l.o.g. $|A| \geq|B| \geq 4$.

Suppose $|A|=3$. Hence $|B|,|\overline{A+B}| \geq 4$ as noted above. If $\left|N_{1}^{b}(A, B)\right|=0$ for all $b \in B$, then Lemma 5.11 - which we can apply to $(A, B)$ in view of Claim 4-implies $|B| \geq|G|-6$, whence $|\overline{A+B}| \leq 3$, a contradiction. Therefore, since $\left|N_{1}^{b}(A, B)\right| \leq 1$ for all $b \in B$ (Claim 8), it follows that we can assume $\left|N_{1}^{b}(A, B)\right|=1$ for some $b \in B$, and w.l.o.g. (by translating $B$ appropriately) we may assume $b \neq 0$. We proceed by induction on $|B|$, with our domain restricted to pairs $\left(A, B^{\prime}\right)$ with each member a non-quasiperiodic, generating subset (the base of the induction is complete in view of Claim 6).

Since $\left|N_{1}^{b}(A, B)\right|=1$, and since $A+B$ is not quasi-periodic (eq. (45)), it follows that $A+(B \backslash b)=(A+B) \backslash \gamma$ is aperiodic with $\gamma \in A+B$. Suppose (17) holds for $A$ and $B \backslash b$. In view of Corollary 4.2, it follows that if the sumset of the extended pair $A \cup\{\alpha\}$ and $B \backslash b \cup\{\beta\}$ is periodic without a unique expression element, then $(A \cup\{\alpha\})+(B \backslash b \cup\{\beta\})=A+(B \backslash b)$. Thus, since $A+(B \backslash b)$ is aperiodic, it follows that we can in all cases apply $\mathrm{KST}$ to $A^{\prime}=A \cup\{\alpha\}$ and $B^{\prime}=B \backslash b \cup\{\beta\}$. Since $d^{\subseteq}(A, \mathcal{Q P}) \geq 2$ (eq. 47), it follows that $d \subseteq\left(A^{\prime}, \mathcal{Q P}\right) \geq 1$. Hence, since $\langle A\rangle=G$ (Claim 4), it follows that KST can only hold with subgroup $G$, whence either $|A+B| \geq\left|A^{\prime}+B^{\prime}\right|-1 \geq|G|-2$, or else $d \subseteq(A, \mathcal{A P}) \leq 1$, both contradictions (to Claim 5 or (51)). Therefore we can assume (17) does not hold. Consequently, the pair $(A, B \backslash b)$ must be non-extendible. Hence, in view of Lemma 5.4 - which we can apply to $(A, B \backslash b)$ in view of Claim $4,|B|,|\overline{A+B}| \geq 4$ and $A+(B \backslash b)$ aperiodic - it follows that $B \backslash b$ is not quasi-periodic and $\langle B \backslash b\rangle=G$. Thus we can apply the induction hypothesis to the pair $(A, B \backslash b)$ and assume (17) does not hold.

Hence, since $\langle A\rangle=G$ and since $A$ is not quasi-periodic (Claim 4), it follows that $H=G$ in Theorem 4.1. Thus, since $|A|,|B \backslash b| \geq 3$, since $|\overline{A+(B \backslash b)}| \geq 4$ (Claim 5), and since $d \subseteq(A, \mathcal{Q P}) \geq 2$ (eq. (47)), it follows that we must have type (VI) with $H=G$ (note type (VII) with $H=G$ would imply $|\overline{A+(B \backslash b)}|=3$, while type (VIII) would imply $d^{\subseteq}(A, \mathcal{Q P})=1$ ), whence $|B|=4$ and w.l.o.g. $A=\{0, d, x\}$ and $B=\{0, d, x, b\}$ with $N_{1}^{b}(A, B)=\{x+b\}$. Since $d^{\subseteq}(A, \mathcal{A P}) \geq 2$ (eq. (51)), then it follows that $x \notin\{2 d, 3 d,-d,-2 d\}$, 
$d \notin\{2 x, 3 x,-x,-2 x\}$ and $0 \notin\{2 x-d, 3 x-2 d, 2 d-x, 3 d-2 x\}$. Additionally, $A$ not quasi-periodic (Claim 4$)$ with $|A|=3$ implies $A$ does not contain a coset of an order two subgroup. Hence the previous two sentences yield that

$$
\{0, d, 2 d\},\{x, x+d\},\{2 x\}
$$

are the three distinct $d$-components of $A+A \subseteq A+B$ : this is because $A+A=\{0, d, x\}+\{0, d, x\}=$ $\{0, d, 2 d, x, x+d, 2 x\}$ while the previous two sentences ensure that all these 6 elements are distinct and that

$$
\{-d, 3 d, x-d, x+2 d, 2 x-d, 2 x+d\}
$$

is disjoint from $\{0, d, 2 d, x, x+d, 2 x\}$. Since $|A+B|=7$, it follows that $b+A$ contains exactly one element distinct from these 6 . Since $N_{1}^{b}(A, B)=\{x+b\}$, it follows that this element must be $x+b$. Thus the component $\{0, d\}+b$ of $b+A$ must be contained in either $\{0, d, 2 d\}$ or $\{x, x+d\}$, whence $b=0, d$ or $x$, all contradictions, completing the induction. So $|A| \geq 4$, and we may w.l.o.g. (in view of Claim 6 ) assume $|A| \geq|B| \geq 4$.

Claim 10: $|\overline{A+B}| \geq 4$.

Suppose $|\overline{A+B}| \leq 3$. Hence $d \subseteq(A+B, \mathcal{P}) \geq 3$ (Claim 5) implies that $|\overline{A+B}|=3$. Thus Corollary 4.3 - which we can apply to $(-B, \overline{A+B})$ in view of Proposition 2.4, Claims 4 and 9, (45) and (46)-implies that $d \subseteq(A, \mathcal{Q P} \cup \mathcal{A P}) \leq 1$, a contradiction to (47) or (51). So we can also assume $|\overline{A+B}| \geq 4$.

If $e+B \subseteq A$ for all $e \in A-B$, then $A-B+B=A$, implying from Kneser's Theorem that $A$ is periodic, a contradiction to (48). Thus we can choose $e \in A-B$ such that $|(e+B) \cap A|$ is maximal subject to $|(e+B) \cap A|<|B|$, and w.l.o.g. (by appropriately translating $A$ and $B$ ) we may assume $e=0$. Let

$$
B(e)=(e+B) \cap A=A \cap B \text { and } A(e)=(e+B) \cup A=A \cup B .
$$

Note that $|A(e)|+|B(e)|=|A|+|B|$, that

$$
A(e)+B(e) \subseteq e+A+B=A+B,
$$

and that $B(e)$ is non-empty. Assume by induction that the theorem holds for non-quasi-periodic, generating subsets $A^{\prime}$ and $B^{\prime}$ either with $\min \left\{\left|A^{\prime}\right|,\left|B^{\prime}\right|\right\}<\min \{|A|,|B|\}=|B|$, or else with $\min \left\{\left|A^{\prime}\right|,\left|B^{\prime}\right|\right\}=$ $\min \{|A|,|B|\}=|B|$ and $\left|A^{\prime}\right|+\left|B^{\prime}\right|>|A|+|B|$ (note $\left|A^{\prime}\right|+\left|B^{\prime}\right|$ is bounded from above by $2|G|$ ). We have already verified the base of the induction when $\min \{|A|,|B|\} \leq 3$.

Claim 11: $B(e)$ is $H$-periodic, where $H=H(A(e)+B(e))$.

Suppose that $B(e)$ is not $H$-periodic, where $H=H(A(e)+B(e))$, and hence $H$ is nontrivial. Then $B(e)$ must have an $H$-hole $x$. Hence, since $A(e)+B(e)$ is $H$-periodic, it follows that

$$
(A \cup B)+(B(e) \cup\{x\})=A(e)+(B(e) \cup\{x\})=A(e)+B(e) \subseteq A+B .
$$

Consequently, $x+A \subseteq A+B$ and $x+B \subseteq A+B$. Since $x$ is an $H$-hole in $B(e)$, then either $x \notin A$ or $x \notin B$. Hence, in view of the last two sentences, it follows that we can contradict the non-extendibility of the pair $(A, B)$ by either adding $x$ to $A$ (if $x \notin A$ ) or else by adding $x$ to $B$ (if $x \notin B$ ). So we may assume that $B(e$ ) is $H$-periodic.

We divide the remainder of CASE 1 into four further subcases.

Subcase 1: $A(e)+B(e)$ is periodic.

Let $H=H(A(e)+B(e))$, let $\rho$ be the number of $H$-holes contained in the pair $A(e)$ and $B(e)$, and let $\rho^{\prime}$ be the number of $H$-holes contained in the pair $A$ and $B$. Partition the set $A$ into the disjoint sets $A \cap B$, $A_{1}$ and $A_{2}$, where $A_{1}=(A \cap(B+H)) \backslash(A \cap B)$, and where $A_{2}$ are the remaining elements of $A$. Likewise 
partition the set $B=(A \cap B) \cup B_{1} \cup B_{2}$. Let $\rho^{\prime \prime}$ be the number of $H$-holes contained among $A_{2}$ and $B_{2}$. Since $A \cap B=B(e)$ is $H$-periodic (Claim 11), it follows that $\phi_{H}\left(A_{1}\right)=\phi_{H}\left(B_{1}\right)$. Hence, since $A_{1} \cap B_{1}$ is empty (by Claim 11), it follows that $\left|A_{1}\right|+\left|B_{1}\right|=\left|A_{1} \cup B_{1}\right| \leq|H|\left|\phi_{H}\left(A_{1}\right)\right|$. Hence, since $A \cap B$ is $H$-periodic (Claim 11), it follows that

$$
\rho=\rho^{\prime \prime}+|H| \cdot\left|\phi_{H}\left(A_{1}\right)\right|-\left|A_{1}\right|-\left|B_{1}\right| \geq \rho^{\prime \prime} .
$$

Applying Kneser's Theorem to $(A(e), B(e))$, it follows that

$$
|A(e)+B(e)| \geq|A|+|B|-|H|+\rho
$$

Suppose that there is some $b^{\prime} \in B_{2}$ with $\left|\phi_{H}\left(B_{b^{\prime}}+A\right) \backslash\left(\phi_{H}(A(e)+B(e))\right)\right|=t \geq 1$. Thus $\rho^{\prime \prime} \geq|H|-\left|B_{b^{\prime}}\right|$. Hence in view of (54) and (53) it follows that

$$
|A+B| \geq|A(e)+B(e)|+t\left|B_{b^{\prime}}\right| \geq|A|+|B|-|H|+\rho^{\prime \prime}+\left|B_{b^{\prime}}\right| \geq|A|+|B|
$$

whence equality holds. However, equality in the above estimate implies $t=1$ and $A+B=(A(e)+B(e)) \cup$ $\left(B_{b^{\prime}}+A\right)$, whence $A+B$ is quasi-periodic, a contradiction to (45). So we can assume $\phi_{H}\left(B_{b^{\prime}}+A\right) \subseteq$ $\phi_{H}(A(e)+B(e))$ for all $b^{\prime} \in B_{2}$, whence the non-extendibility of $B$ implies $B_{2}$ is $H$-periodic (or empty). By the same argument applied to $a^{\prime} \in A_{2}$ it follows that $\phi_{H}\left(A_{a^{\prime}}+B\right) \subseteq \phi_{H}(A(e)+B(e))$ for all $a^{\prime} \in A_{2}$, and that $A_{2}$ is $H$-periodic (or empty). Consequently $\rho^{\prime \prime}=0$.

Let $A_{1}=A_{\alpha_{1}} \cup \ldots A_{\alpha_{n}}$ and $B_{2}=B_{\beta_{1}} \cup \ldots B_{\beta_{n}}$ be $H$-coset decompositions of $A_{1}$ and $B_{1}$, with $\phi_{H}\left(\alpha_{i}\right)=$ $\phi_{H}\left(\beta_{i}\right)$. In view of the result of the previous paragraph, Claim 11 and Lemma 5.6 - which we can apply to $(A, B)$ in view of Claims 3,4 and 5 -it follows that $n \geq 3$, else the proof is complete.

Note that $e^{\prime} \in A_{\alpha_{i}}-B_{\beta_{i}} \subseteq H$ are exactly those elements such that $\left(e^{\prime}+B_{\beta_{i}}\right) \cap A_{\alpha_{i}}$ is nonempty. Additionally, since $A \cap B$ is $H$-periodic (Claim 11), and since $e^{\prime} \in H$, it follows that

$$
\left(\left(e^{\prime}+B_{\beta_{i}}\right) \cap A_{\alpha_{i}}\right) \cup(A \cap B) \subseteq A \cap\left(e^{\prime}+B\right) .
$$

Thus, in view of the previous two sentences, unless $e^{\prime}+B \subseteq A$, then the element $e^{\prime}$ will contradict the maximality of $e=0$. Hence in order to avoid this contradiction we must have: (a) $B_{2}$ empty (else w.l.o.g. there will be an $H$-coset $\beta+H$ which intersects $e^{\prime}+B$ but not $A$ ), and (b) $e^{\prime}+B_{\beta_{i}} \subseteq A_{\alpha_{i}}$ for each $e^{\prime} \in A_{\alpha_{i}}-B_{\beta_{i}}$ (else w.l.o.g. there will be an element from the coset $\alpha_{i}+H$ contained in $e^{\prime}+B$ but not in $A^{\prime}$ ), and (c) $A_{\alpha_{i}}-B_{\beta_{i}}=A_{\alpha_{j}}-B_{\beta_{j}}$ for all $i$ and $j$ (else w.l.o.g. there will be an element $e^{\prime} \in A_{\alpha_{i}}-B_{\beta_{i}}$ but $e^{\prime} \notin A_{\alpha_{j}}-B_{\beta_{j}}$, whence the elements from the coset $e^{\prime}+\alpha_{j}+H$ contained in $e^{\prime}+B$ will not be contained in $A$, but some element from the coset $e^{\prime}+\alpha_{i}+H$ contained in $e^{\prime}+B$ will be contained in $A$ ).

Since $e^{\prime}+B_{\beta_{i}} \subseteq A_{\alpha_{i}}$ for each $e^{\prime} \in A_{\alpha_{i}}-B_{\beta_{i}}$, it follows that $A_{\alpha_{i}}-B_{\beta_{i}}+B_{\beta_{i}}=A_{\alpha_{i}}$, implying that $B_{\beta_{i}}-B_{\beta_{i}} \subseteq H\left(A_{\alpha_{i}}\right)$, where $A_{\alpha_{i}}$ is maximally $H\left(A_{\alpha_{i}}\right)$-periodic. Hence $A_{\alpha_{i}}-B_{\beta_{i}}=-\beta_{i}+A_{\alpha_{i}}$. Thus, since $A_{\alpha_{i}}-B_{\beta_{i}}=A_{\alpha_{j}}-B_{\beta_{j}}$ for all $i$ and $j$, it follows that $A_{\alpha_{i}}=A_{\alpha_{j}}+\left(\beta_{i}-\beta_{j}\right)$. Consequently, the $A_{\alpha_{i}}$ are all just translates of one another, implying that $H\left(A_{\alpha_{i}}\right)=H\left(A_{\alpha_{j}}\right)=H^{\prime} \leq H$, and that $\left|\phi_{H^{\prime}}\left(B_{\beta_{i}}\right)\right|=1$ (whence $\left.\left|B_{\beta_{i}}\right| \leq\left|H^{\prime}\right|\right)$, for all $i$ and $j$. Note $H^{\prime}$ must be a proper subgroup of $H$, else $A_{\alpha_{i}} \cap B_{\beta_{i}}$ would be nonempty, a contradiction. Thus, since $A_{2}$ is $H$-periodic (or empty), it follows that $A$ is $H^{\prime}$-periodic, whence $\left|H^{\prime}\right|=1$. Hence $B_{\beta_{i}}-B_{\beta_{i}} \subseteq H\left(A_{\alpha_{i}}\right)=H^{\prime}$ implies that $\left|B_{\beta_{i}}\right|=1$ for all $i$.

For each partially filled $H$-coset $F_{i}$ in $A+B$, it follows that there must be at least one pair $A_{\alpha_{i}}$ and $B_{\beta_{j}}$ such that $A_{\alpha_{i}}+B_{\beta_{j}} \subseteq F_{i}$. Since $A+B$ is not quasi-periodic (eq. (45)), it follows (in view of $\left|\phi_{H}(A+B)\right| \geq n \geq 3$ ) that there are at least two distinct partially filled $H$-cosets in $A+B$. In view of the non-extendibility of $A$, it follows that each $A_{\alpha_{i}}$ must have a $B_{\beta_{\sigma(i)}}$ such that $A_{\alpha_{i}}+B_{\beta_{\sigma(i)}} \subseteq F_{j}$ for some $j$. Likewise for each $B_{b_{i}}$. Hence in view of Proposition 5.5 and $n \geq 3$, it follows that there exist distinct $i$ and $i^{\prime}$ and distinct $j$ and $j^{\prime}$ 
such that $A_{\alpha_{i}}+B_{\beta_{j}}$ and $A_{\alpha_{i^{\prime}}}+B_{\beta_{j^{\prime}}}$ are each disjoint from $A(e)+B(e)$ and $\phi_{H}\left(\alpha_{i}+\beta_{j}\right) \neq \phi_{H}\left(\alpha_{i^{\prime}}+\beta_{j^{\prime}}\right)$. Thus in view of (54) it follows that

$$
|A+B| \geq|A(e)+B(e)|+\left|A_{\alpha_{i}}+B_{\beta_{j}}\right|+\left|A_{\alpha_{i^{\prime}}}+B_{\beta_{j^{\prime}}}\right| \geq|A|+|B|+\rho+\left|A_{\alpha_{i}}\right|+\left|A_{\alpha_{i^{\prime}}}\right|-|H|,
$$

with equality possible only if there are exactly two partially filled $H$-cosets in $A+B$.

Since $\left|B_{\beta_{i}}\right|=1$ for all $i$, and since $\rho^{\prime \prime}=0$, it follows in view of (53) that $\rho=n(|H|-1)-\sum_{i=1}^{n}\left|A_{\alpha_{i}}\right| \geq$ $2(|H|-1)-\left|A_{\alpha_{i^{\prime}}}\right|-\left|A_{\alpha_{i}}\right|$. Thus (55) implies $|A+B| \geq|A|+|B|+|H|-2$, whence $|H|=2$ and equality must hold in (55). Hence there are exactly two partially filled $H$-cosets in $A+B$. Thus, since $|H|=2$ implies each partially filled $H$-coset contains one hole, it follows that $d \subseteq\left(A+B, \mathcal{P}_{H}\right) \leq 2$, contradicting that $d \subseteq(A+B, \mathcal{P}) \geq 3$ (Claim 5). So we may assume $A(e)+B(e)$ is aperiodic.

Since $A(e)+B(e)$ is aperiodic, in view of Subcase 1, it follows from Kneser's Theorem that either

$$
|A(e)+B(e)|=|A(e)|+|B(e)|-1 \quad \text { or } \quad|A(e)+B(e)|=|A(e)|+|B(e)| .
$$

We proceed based on $|B(e)|$.

Subcase 2: $|B(e)| \geq 3$.

Suppose $|A(e)+B(e)|=|A(e)|+|B(e)|-1$. Thus we can apply KST to $A(e)+B(e)=(A+B) \backslash \gamma$ with $\gamma \in A+B$. Hence, since $\left\langle-\gamma^{\prime}+\overline{A+B}\right\rangle=G$ for $\gamma^{\prime} \in \overline{A+B}$ (eq. (46)), and since $d^{\subseteq}(\overline{A+B}, \mathcal{Q P}) \geq 2$ (eq. (49)), it follows that the quasi-period from KST must be $G$. Hence from KST it follows that either $d \subseteq(\overline{A+B}, \mathcal{A P}) \leq 1$ or else $|A+B| \geq|G|$, both contradictions (to (51) or Claim 10). So we can assume instead that $A(e)+B(e)=A+B$ (in view of (56) and (52)).

Suppose $(A(e), B(e))$ is extendible. Hence we can apply KST to $(A(e) \cup\{\alpha\})+(B(e) \cup\{\beta\})=A+B$. As in the previous paragraph, the quasi-period from KST must be $G$, whence either $d \subseteq(\overline{A+B}, \mathcal{A P}) \leq 0$ or else $|A+B| \geq|G|-1$, both contradictions (to (51) or Claim 10). So we can assume $(A(e), B(e)$ ) is non-extendible. Thus Lemma 5.4-which we can apply to $(-\gamma+\overline{A+B},-B(e))$ and $(-\gamma+\overline{A+B},-A(e))$, where $\gamma \in \overline{A+B}$, in view of Proposition 2.4 (recall $A(e)+B(e)=A+B$ ), Claim 10, $|A(e)| \geq|B(e)| \geq 3$, (45), (46) and $A(e)+B(e)$ aperiodic - implies that $A(e)$ and $B(e)$ are both non-quasi-periodic, generating subsets, whence the theorem holds for $A(e)$ and $B(e)$ by induction hypothesis (since $|B(e)|<|B| \leq|A|$ and $|A|+|B|=|A(e)|+|B(e)|)$. Hence in view of Corollary 4.3 - which we can apply to $(A(e), B(e))$ in view of $|A(e) \geq| B(e) \mid \geq 3$, and Claims 9 and 10-it follows that $d \subseteq(\overline{A+B}, \mathcal{Q P} \cup \mathcal{A P}) \leq 1$ (recall $A(e)+B(e)=A+B$ ), a contradiction (to (49) or (51)).

Subcase 3: $|B(e)|=1$.

Let $T$ be the subset of $A-B$ such that $T+B \subseteq A$. Since $|B(e)|=1$, the maximality of $e$ implies that $|(x+B) \cap A| \leq 1$ for all $x \in G \backslash T$, which implies $r_{A,-B}(x) \leq 1$ for all $x \in G \backslash T$. Thus we can apply Theorem 3.1 with $k=1$, whence Theorem 3.1(ii) implies

$$
|T| \geq|A| \frac{|A||B|-|A|-|B|}{(|A|+|B|)(|B|-1)} .
$$

If $|T| \leq 1$, then (57) implies $(|B|-1)|A|^{2}-(2|B|-1)|A|-|B|(|B|-1) \leq 0$. This is an increasing function of $|A|$ for $|A|>\frac{2|B|-1}{2|B|-2}$, whence $|A| \geq|B| \geq 4$ (Claim 9) implies

$$
0 \geq(|B|-1)|B|^{2}-(2|B|-1)|B|-|B|(|B|-1)=|B|\left(|B|^{2}-4|B|+2\right),
$$


contradicting that $|B| \geq 4$ (Claim 9). Therefore we can assume $|T| \geq 2$. If $|T| \leq|A|-|B|-2$, then (57) implies, in view of $|B| \geq 4$ (Claim 9), that $|A| \leq \frac{2|B|-|B|^{3}-|B|^{2}}{|B|-2}<0$, a contradiction. Therefore we can assume

$$
|T| \geq|A|-|B|-1 \text {. }
$$

Let $A^{\prime}=A \backslash(T+B)$. Hence $A=A^{\prime} \cup(T+B)$ and $|T+B|=|A|-\left|A^{\prime}\right|$. Let $a^{\prime} \in A^{\prime}$ and $a \in A$. If $\left(a^{\prime}+B\right) \cap(a+B)$ is nonempty, then $a^{\prime}+b^{\prime}=a+b$ for some $b, b^{\prime} \in B$. Hence $a^{\prime} \in\left(a^{\prime}-b\right)+B$ and $a=a^{\prime}-b+b^{\prime} \in\left(a^{\prime}-b\right)+B$. Hence, if $a^{\prime}$ and $a$ are distinct, then $\left|\left(a^{\prime}-b+B\right) \cap A\right| \geq 2$, whence $a^{\prime}-b+B \subseteq A$. Thus $a^{\prime}-b \in T$ and $a^{\prime} \in T+B$, a contradiction. Therefore every element $a^{\prime}+b$, for $a^{\prime} \in A^{\prime}$ and $b \in B$, is a unique expression element in $A+B$. Hence

$$
|A+B| \geq\left|A^{\prime}\right||B|+\left|\left(A \backslash A^{\prime}\right)+B\right| \geq|A|+\left|A^{\prime}\right|(|B|-1)=|A|+|B|+\left(\left|A^{\prime}\right|-1\right)(|B|-1)-1 .
$$

Thus in view of $|A+B|=|A|+|B|$ and $|B| \geq 3$ (Claim 9), it follows that $\left|A^{\prime}\right| \leq 1$. As a result, since $d \subseteq(A, \mathcal{Q P}) \geq 2$ (eq. (47)), it follows that $T+B$ is aperiodic.

Suppose $r_{B,-B}(b) \geq 2$ for some nonzero $b$. Hence $|(b+t+B) \cap(t+B)| \geq 2$ for $t \in T$. Thus, since $t+B \subseteq A$, it follows that $|(b+t+B) \cap A| \geq 2$, whence $(b+t+B) \subseteq A$. Consequently, $b+T+B \subseteq A$, whence the definition of $T$ implies that $\{0, b\}+T=T$. Thus, since $b$ is nonzero, it follows in view of Kneser's Theorem that $T$ is periodic, contradicting that $T+B$ is aperiodic. So we can assume $r_{B,-B}(b) \leq 1$ for all nonzero $b$. Consequently, $B$ is a Sidon set and $|2 B|=\frac{|B|(|B|+1)}{2}$.

Since $\left|A^{\prime}\right| \leq 1$, it follows that $T+B=A \backslash \alpha$ for some $\alpha \in G$, whence $T+2 B=(A \backslash \alpha)+B$. Hence, since $\left|N_{1}^{a}(B, A)\right| \leq 1$ for all $a \in A$ (Claim 8), it follows that $T+2 B=(A+B) \backslash \gamma$ for some $\gamma \in G$. Thus $A+B$ not quasi-periodic (eq. (45)) implies that $T+2 B$ is aperiodic.

If the inequality in (58) is strict, then since $T+2 B$ is aperiodic, it follows from Kneser's Theorem that $|A|+|B| \geq|T+2 B| \geq|T|+|2 B|-1=|A|-|B|+\frac{|B|(|B|+1)}{2}-1$, implying $|B|<4$, a contradiction to Claim 9. Therefore we can assume $|T|=|A|-|B|-1$. Hence (57) implies that

$$
|A| \geq|B|(|B|-1)(|B|+1) \text {. }
$$

Note (where $M$ is as defined in Theorem 3.1) that

$$
-\frac{M}{|B|}=-|T|(|B|-1)=(|B|-|A|+1)(|B|-1) \equiv(|B|+1)(|B|-1) \quad \bmod |A| .
$$

In view of $(59)$, it follows that $1 \leq(|B|+1)(|B|-1) \leq|A|$. Hence in view of (60) it follows that $x=$ $(|B|+1)(|B|-1)|B|$ in Theorem 3.1. Thus Proposition 3.1(iii) and $|A+B|=|A|+|B|$ imply

$$
|A|+|B|=|A+B| \geq \frac{|A|^{2}|B|^{2}\left(|A||B|^{2}-|A||B|+|B|^{3}-|B|\right)}{|A||B|^{2}\left(|A||B|^{2}-|A||B|\right)}=\frac{|A||B|-|A|+|B|^{2}-1}{|B|-1},
$$

which implies $|B| \leq 1$, a contradiction to Claim 9 .

Subcase 4: $|B(e)|=2$.

If $|A(e)+B(e)|=|A(e)|+|B(e)|-1$, then the arguments from the analogous part of Subcase 2 complete the proof. Therefore we can assume $|A(e)+B(e)|=|A(e)|+|B(e)|, A(e)+B(e)=A+B$ and $c_{d}(A(e))=2$, for $d$ equal to the difference of elements in $B(e)$ (in view of (56) and (52)). Thus $A(e)+B(e)=A+B$ implies that $c_{d}(A+B)=c_{d}(\overline{A+B}) \leq c_{d}(A(e))=2$. If $c_{d}(\overline{A+B})=1$, then $\overline{A+B}$ not quasi-periodic (eq. (45)) implies $\overline{A+B}$ is an arithmetic progression, contradicting (51). Therefore $c_{d}(\overline{A+B})=2$. Thus, in view of $d^{\subseteq}(B, \mathcal{Q P} \cup \mathcal{A P}) \geq 2$ (eq. (47) and (51)) and Lemma 5.9-which we can apply to $(-\gamma+\overline{A+B},-B)$, where $\gamma \in \overline{A+B}$, in view of Proposition 2.4, Claims 9 and 10, (45), (46) and (48)-it follows that $c_{d}(A), c_{d}(B) \leq 2$. Furthermore, we must have $c_{d}(A)=c_{d}(B)=2$ by the same reasoning used to establish this for $\overline{A+B}$. 
Since $d \subseteq(A+B, \mathcal{P}) \geq 3$ (Claim 5) and $c_{d}(A+B)=2$, it follows that

$$
d^{\subseteq}(A+B+\{0, d\}, \mathcal{P}) \geq 1 .
$$

Suppose $d^{\subseteq}(A+B+\{0, d\}, \mathcal{P})=1$. Thus by the arguments used to establish the theorem when $d \subseteq(A+$ $B, \mathcal{P})=1$ (Claim 2), it follows that (17) holds for $A$ and $B+\{0, d\}$ with $(A \cup\{\alpha\})+((B+\{0, d\}) \cup\{\beta\})$ periodic with maximal period (say) $H$. If $(A \cup\{\alpha\})+((B+\{0, d\}) \cup\{\beta\})$ contains no unique expression element, then Corollary 4.2 implies that $(A \cup\{\alpha\})+((B+\{0, d\}) \cup\{\beta\})=A+B+\{0, d\}$. Hence, since $d \subseteq(A+B+\{0, d\}, \mathcal{P}) \geq 1$, it follows that we can assume this does not happen, whence we can apply KST to $(A \cup\{\alpha\})+((B+\{0, d\}) \cup\{\beta\})$. Since $d^{\subseteq}(A, \mathcal{Q P}) \geq 2$ (eq. (47)), and since $\langle A\rangle=G$ (Claim 4), it follows that the quasi-period from KST must be $G$, whence $(A \cup\{\alpha\})+((B+\{0, d\}) \cup\{\beta\})$ periodic and $c_{d}(A+B)=2$ imply that $|\overline{A+B}| \leq 3$, a contradiction to Claim 10. So we can assume

$$
d^{\subseteq}(A+B+\{0, d\}, \mathcal{P}) \geq 2 .
$$

If the pair $(A+\{0, d\}, B)$ is extendible, then since $d \subseteq(B, \mathcal{Q P}) \geq 2$ (eq. (47)), since $\langle B\rangle=G$ (Claim 4 ), and since $d \subseteq(A+B+\{0, d\}, \mathcal{P}) \geq 2$ (eq. (61)), it follows in view of KST that $d \subseteq(B, \mathcal{A P}) \leq 1$, a contradiction to (51). Therefore we can assume $(A+\{0, d\}, B)$ is non-extendible. Thus, since $B$ is not quasi-periodic and since $\langle B\rangle=G$ (Claim 4), it follows in view of Lemma 5.3-which we can apply to $(A+\{0, d\}, B)$ in view of (61) - that $A+\{0, d\}$ is not quasi-periodic. Also, $\langle A+\{0, d\}\rangle \geq\langle A\rangle=G$ (Claim 4) implies $\langle A+\{0, d\}\rangle=G$. Thus we can apply the induction hypothesis to the pair $(A+\{0, d\}, B)$. Since $|A+\{0, d\}|,|B| \geq 4$ (Claim 9 ), with neither $A+\{0, d\}$ nor $B$ quasi-periodic (the former by Claim 4, while the latter we have just seen), it follows that we cannot have type (V)-(VII). Since $d^{\subseteq}(B, \mathcal{Q P}) \geq 2$ (eq. (47)), it follows that we cannot have type (VIII). Thus (17) holds for $A+\{0, d\}$ and $B$. Since $\langle B\rangle=G$ (Claim 4 ), and since $d \subseteq(B, \mathcal{Q P}) \geq 2$ (eq. (47)), it follows that the quasi-period from KST must be $G$. Hence, since $d^{\subseteq}(B, \mathcal{A P}) \geq 2$ (eq. (51)), it follows in view of KST that we must have type (III) or (IV) with $H=G$, where $H$ is from the definition of (III) and (IV), whence $|\overline{A+B+\{0, d\}}| \leq 2$ (recall KST was applied to the extended pair given by (17)), which together with $c_{d}(A+B)=2$ implies $|\overline{A+B}| \leq 4$, and thus $|\overline{A+B}|=4$ (by Claim 10).

Suppose $|B| \geq 5$. Hence we can apply the induction hypothesis to $(-A, \overline{A+B}$ ) (in view of $|\overline{A+B}|=4<$ $|B| \leq|A|$, Claim 4, (45) and (46)). Thus, since $d \subseteq(A, \mathcal{Q P}) \geq 2$ (eq. (47)), it follows in view of Corollary 4.3- which we can apply to $(-A, \overline{A+B})$ in view of Proposition 2.4, Claims 4, 9 and 10, (45) and (46) - that $d \subseteq(-A, \mathcal{A P})=d^{\subseteq}(A, \mathcal{A P}) \leq 1$, a contradiction to (51). So we can assume $|B|=|\overline{A+B}|=4$. Note that if the theorem holds for $(-B, \overline{A+B})$, then it follows in view of $d \subseteq(B, \mathcal{Q P}) \geq 2$ (eq. (47)) and Corollary 4.3 - which we can apply to $(-B, \overline{A+B})$ in view of Claims 4,9 and $10,(45)$ and $(46)$ - that $d \subseteq(-B, \mathcal{A P})=$ $d^{\subseteq}(B, \mathcal{A P}) \leq 1$, a contradiction to (51). Consequently, since $|B|=|\overline{A+B}|=4$, it follows that case $G$ finite will be complete once we complete the case with $|A|=|B|=4$ and $c_{d}(A)=c_{d}(B)=c_{d}(A+B)=2$ (as then we can apply this case with $-B$ and $\overline{A+B}$ ). We proceed to do so.

Suppose $\left|N_{1}^{b}(A, B)\right|>0$ for some $b \in B$, and w.l.o.g. (by translating $B$ appropriately) assume $b \neq 0$. Hence in view of $\left|N_{1}^{b}(A, B)\right| \leq 1$ (by Claim 8), it follows that $\left|N_{1}^{b}(A, B)\right|=1$. If the pair $(A, B \backslash b)$ is extendible, then the theorem holds for the pair $(A, B \backslash b)$. Otherwise, it follows from Lemma 5.4 -which we can apply to $(A, B \backslash b)$ in view of Claims 4,9 and 10 and (45) - that $B \backslash b$ is not quasi-periodic and $\langle B \backslash b\rangle=G$, whence the theorem holds for the pair $(A, B \backslash b)$ by induction hypothesis. In the latter case, Corollary 4.3 - which we can apply to $(A, B \backslash b)$ in view of Claims 4, 9 and 10-implies $d \subseteq(A, \mathcal{A P} \cup \mathcal{Q P}) \leq 1$, a contradiction (to (47) or (51)). In the former case, we can apply KST to $A \cup\{\alpha\}$ and $B \cup\{\beta\}$. Since $d^{\subseteq}(A, \mathcal{Q P}) \geq 2$ (eq. (47)), and since $\langle A\rangle=G$ (Claim 4), it follows that the quasi-period must be $G$, whence $|\overline{A+B}|=4$ likewise implies that $d^{\subseteq}(A, \mathcal{A P}) \leq 1$, again a contradiction to (51). So we can assume there are no unique expression elements in $A+B$ (i.e., $\left|N_{1}^{b}(A, B)\right|=0$ for all $b \in B$ ). 
Let $A_{1}$ and $A_{2}$ be the two $d$-components of $A$, and let $B_{1}$ and $B_{2}$ be the two $d$-components of $B$. Suppose for some $i$, say $i=1$, that $\left|A_{1}\right| \geq 3$. Hence in view of $d^{\subseteq}\left(B, \mathcal{Q} \mathcal{A} \mathcal{P}_{d}\right) \geq 2$ (eq. (50)), it follows that $\left|A_{1}+B\right|=|B|+4=|B|+|A|$. Thus $A_{1}+B=A+B$. Since $c_{d}(A+B)=2$, this implies that $A_{1}+B_{1}$ and $A_{1}+B_{2}$ are distinct components in $A+B$, and that each of the four end terms of components in $A_{1}+B$ is a unique expression element. We may assume w.l.o.g. that $\left|B_{1}\right| \geq\left|B_{2}\right|$. Thus the component $A_{1}+B_{1}$ is longer than either of the components $A_{2}+B_{1}$ and $A_{2}+B_{2}$. Hence, since $A+B$ contains no unique expression element, and since $A_{1}+B=A+B$, it follows that the only way the two end terms of $A_{1}+B_{1}$ will not be unique expression elements in $A+B$ is if $A_{2}+B \subseteq A_{1}+B_{1}$. Thus both the end terms of $A_{1}+B_{2}$ are unique expression elements, a contradiction. So we can assume $\left|A_{i}\right|=2$ for $i=1,2$. Likewise $\left|B_{i}\right|=2$ for $i=1,2$.

Since $d \subseteq\left(B, \mathcal{Q A P}_{d}\right) \geq 2$ (eq. (50)), it follows that $A_{1}+B_{1}$ and $A_{1}+B_{2}$ are distinct components in $A_{1}+B$, and that all four of the end terms are unique expression elements in $A_{1}+B$. Since $\left|A_{i}+B_{j}\right|=\left|A_{i^{\prime}}+B_{j^{\prime}}\right|$ for all $i, i^{\prime}, j, j^{\prime}$, and since $c_{d}(A+B)=2$, it follows that only way that these four terms can all not be unique expression elements in $A+B$ is if $A_{2}+B_{1}=A_{1}+B_{2}$ and $A_{2}+B_{2}=A_{1}+B_{1}$. Thus $A_{1}+B=A_{2}+B$, implying $|A+B|=|B|+2<|A|+|B|$, a contradiction. Consequently, we conclude that Theorem 4.1 holds for $G$ finite.

\section{CASE II: $G$ is Infinite.}

Assume $G$ is infinite. Since $A$ and $B$ are finite, we may w.l.o.g. assume $G$ is finitely generated. Hence $G \cong$ $\mathbb{Z}^{l} \times T$, where $T$ is the torsion subgroup of $G$. By translation, we can assume all non-torsion coordinates for all $a \in A$ and $b \in B$ are non-negative. Let $M$ be the maximum integer that occurs in a non-torsion coordinate of the $a \in A$ and $b \in B$. Let $p$ be a prime such that $p>4(M+|T|+|A||B|)$. Let $\varphi: G \cong \mathbb{Z}^{l} \times T \rightarrow(\mathbb{Z} / p \mathbb{Z})^{l} \times T$ be the map defined by reducing all non-torsion coordinates modulo $p$. Since $p>4(M+|T|+|A||B|) \geq 2 M$, it follows that $\varphi$ is a Freiman isomorphism of $(A+T, B+T)$ (see the definition given before the start of the proof of Theorem 4.1), and thus also of $(A, B)$. Hence $|\varphi(A+B)|=|\varphi(A)|+|\varphi(B)|=|A|+|B|$. Thus, since $\varphi(G)$ is finite, it follows that we can apply Theorem 4.1 to $\varphi(A)$ and $\varphi(B)$.

If $d \subseteq\left(\varphi(A+B), \mathcal{P}_{H}\right) \leq 2$, for some nontrivial subgroup $H \leq \varphi(G)$, then

$$
|A|+|B|=|\varphi(A+B)| \geq|H|-2 .
$$

Hence $p>4(M+|T|+|A||B|) \geq 4(|A||B|+1)$ implies $H \leq T$ (since any element outside $T$ has a coordinate with order at least $p$, and thus is itself of order at least $p$ ). Hence from the definition of $\varphi$ it follows that $d \subseteq\left(A+B, \mathcal{P}_{H}\right) \leq 2$, a contradiction. Therefore we can assume $d \subseteq(\varphi(A+B), \mathcal{P}) \geq 3$. Since $\langle A\rangle=G$, it follows that $\langle\varphi(A)\rangle=\varphi(G)$. Likewise $\langle\varphi(B)\rangle=\varphi(G)$.

Suppose that $d^{\subseteq}\left(\varphi(A), \mathcal{Q P} \mathcal{P}_{H}\right)=0$ for some nontrivial subgroup $H \leq \varphi(G)$. Hence $|\varphi(A)|=|A| \geq|H|$. Thus, since $p>4(M+|T|+|A||B|) \geq 4(|A||B|+1)$, it follows that $H \leq T \leq G$. Hence $d^{\subseteq}(\varphi(A), \mathcal{Q P} H)=0$ implies (in view of the definition of $\varphi$ ) that $A$ is quasi-periodic with quasi-period $H \leq G$, a contradiction. So we can assume $d \subseteq(\varphi(A), \mathcal{Q P}) \geq 1$. Likewise $d \subseteq(\varphi(B), \mathcal{Q P}) \geq 1$.

Suppose

$$
d^{\subseteq}\left(\varphi(A), \mathcal{A P}_{d}\right), d^{\subseteq}(\varphi(B), \mathcal{A P} d) \leq 1
$$

for some nonzero $d \in \varphi(G)$. Consequently, $\varphi(G)=\langle\varphi(A)\rangle$ is cyclic, implying $G \cong \mathbb{Z} \times T$ with $T$ cyclic. For $x \in \varphi(G)$, let $\bar{x}$ denote the least non-negative integer representative of the integer coordinate of $x$.

If $d \in T$, then it follows that $\varphi(A) \subseteq T$, whence $A \subseteq T$, contradicting that $\langle A\rangle=G$ is infinite. Therefore we can assume $d \notin T$. Hence, by considering $-d$ if needed, it follows that $1 \leq \bar{d} \leq \frac{p-1}{2}$. Since $d \subseteq(\varphi(A), \mathcal{A P} d) \leq$ 1 , let $P=\left\{p_{0}, p_{0}+d, \ldots, p_{0}+|A| d\right\}$ be an arithmetic progression with difference $d$ that contains $\varphi(A)$.

Suppose $\overline{p_{0}+i d}=\overline{p_{0}}+i \bar{d}$ for all $i$ does not hold. Hence, if $d \leq M$, then $p>4(M+|T|+|A||B|)>4 M$ implies that $\left\{\overline{p_{0}}, \overline{p_{0}+d}, \ldots, \overline{p_{0}+|A| d}\right\}$ must contain at least two elements from the interval $(M, p)$. Hence 
$P$ contains at least two elements from $\bar{A}$, contradicting the $|P \backslash A| \leq 1$. Otherwise, $d \leq \frac{p-1}{2}$ and $M \leq \frac{p-1}{2}$ imply that $\left\{\overline{p_{0}}, \overline{p_{0}+d}, \ldots, \overline{p_{0}+|A| d}\right\}$ contains at least $|A|-1$ elements from the interval $(M, p)$, whence $|P \backslash A| \leq 1$ implies $|A| \leq 2$, a contradiction. So we may assume $\overline{p_{0}+i d}=\overline{p_{0}}+i \bar{d}$ for all $i$.

Hence $A$ is contained in an arithmetic progression of difference $(\bar{d}, t)$ and at most one hole, where $t$ is the torsion coordinate of $d$. By the same argument applied to $B$, it follows that $B$ is also contained in an arithmetic progression of difference $(\bar{d}, t)$ with at most one hole. Hence letting $\alpha$ be the hole in $A$, and letting $\beta$ be the hole in $B$, it follows in view of $|A+B|=|A|+|B|$ that (17) holds, completing the proof. So we may assume that $(62)$ does not hold.

Suppose that $(\varphi(A), \varphi(B))$ is extendible. Hence w.l.o.g. there exists $\alpha \in \overline{\varphi(A)}$ such that $(\varphi(A) \cup\{\alpha\})+$ $\varphi(B)=\varphi(A)+\varphi(B)$. Thus we can apply KST to $(\varphi(A) \cup\{\alpha\}, \varphi(B))$. Since $d \subseteq(\varphi(B), \mathcal{Q P}) \geq 1$, and since $\langle\varphi(B)\rangle=\varphi(G)$, it follows that the quasi-period from KST must be $\varphi(G)$. Hence, since $d \subseteq(\varphi(A+B), \mathcal{P}) \geq 3$, it follows from KST that (62) holds, a contradiction. So we can assume $(\varphi(A), \varphi(B))$ is non-extendible.

Since $(\varphi(A), \varphi(B))$ is non-extendible, since $|\overline{\varphi(A+B)}|,|\varphi(B)| \geq 4$, and since (62) does not hold, it follows in view of Corollary 4.3 that $d^{\subseteq}\left(\varphi(A), \mathcal{Q P}_{H}\right)=1$ for some nontrivial subgroup $H \leq \varphi(G)$. Hence $|\varphi(A)|=|A| \geq|H|-1$. Thus, since $p>4(M+|T|+|A||B|) \geq 4(|A||B|+1)$, it follows that $H \leq T \leq G$. Observe that we have verified all the hypotheses needed to apply Lemma 5.7 to $(\varphi(A), \varphi(B))$. Hence Lemma 5.7 implies that (17) holds for $(\varphi(A), \varphi(B))$. Thus, since $\varphi$ is a Freiman isomorphism for $(A+T, B+T)$, since $H \leq T$, and since the proof of Lemma 5.7 shows $\alpha \in \varphi(A)+H$ and $\beta \in \varphi(B)+H$, it follows that (17) holds for $(A, B)$, completing the proof.

Acknowledgements: I wish to thank Oriol Serra, Alfred Geroldinger and the referee for some helpful comments regarding the manuscript, including the simplified proof of Proposition 5.5, as suggested by the referee.

\section{REFERENCES}

[1] Y. F. Bilu, V. F. Lev, and I. Z. Ruzsa, Rectification principles in additive number theory, Dedicated to the memory of Paul Erdős, Discrete Comput. Geom., 19 (1998), no. 3, Special Issue, 343-353.

[2] S. Chowla, A theorem on the addition of residue classes: applications to the number $\Gamma(k)$ in Warings problem, Proc. Indian Acad. Sc., 2 (1935), 242-243.

[3] J. Deshouillers and G. A. Freiman, A step beyond Kneser's theorem for abelian finite groups, Proc. London Math. Soc., (3) 86 (2003), no. 1, 1-28.

[4] G. A. Freiman, Inverse problems of additive number theory, On the addition of sets of residues with respect to a prime modulus, Doklady Akad. Nauk SSSR, 141 (1961), 571-573.

[5] G. A. Freiman, Inverse problems of additive number theory, On the addition of sets of residues with respect to a prime modulus, Soviet Math.-Doklady, 2 (1961), 1520-1522.

[6] G. A. Freiman, Foundations of a structural theory of set addition, translated from the Russian, Translations of Mathematical Monographs, Vol 37, American Mathematical Society, Providence, RI, 1973.

[7] G. A. Freiman, The addition of finite sets I. (Russian), Izv. Vyš̌. Učebn. Zaved. Matematika (1959), no. 6 (13), $202-213$.

[8] G. A. Freiman, Inverse problems of additive number theory VI: On the addition of finite sets III, (Russian) Izv. Vys . S.U.cebn. Zaved. Matematika (1962), no. 3 (28), 151-157

[9] D. J. Grynkiewicz, An extension of the Erdős-Ginzburg-Ziv Theorem to hypergraphs, European J. Combin., 26 (2005), no. 8, 1154-1176.

[10] D. J. Grynkiewicz, Quasi-periodic decompositions and the Kemperman structure theorem, European J. Combin., 26 (2005), no. $5,559-575$.

[11] D. J. Grynkiewicz, Sumsets, zero-sums and extremal combinatorics, Ph.D. Dissertation, Caltech (2006).

[12] D. J. Grynkiewicz, V. Lev, and O. Serra, Connectivity of addition Cayley graphs, to appear in J. Combin. Theory, Ser. B.

[13] D. J. Grynkiewicz, E. Marchan, and O. Ordaz, Representation of finite abelian group elements by subsequence sums, preprint.

[14] D. J. Grynkiewicz and O. Serra, The Freiman $3 k-2$ Theorem: Distinct Summands, in preparation. 
[15] D. J. Grynkiewicz, On Extending Pollard's Theorem for $t$-Representable Sums, prepreint.

[16] Y. O. Hamidoune, O. Serra, and G. Zemor, On the Critical Pair Theory in Abelian Groups: Beyond Chowla's Theorem, Combinatorica, 28 (2008), no. 4, 441-467.

[17] Y. O. Hamidoune, O. Serra, and G. Zemor, On the critical pair theory in $\mathbb{Z} / p \mathbb{Z}$, Acta Arith., 121 (2006), no. 2.

[18] Y. O. Hamidounee, A. Montejano and O. Serra, Rainbow-free Three Colorings in Abelian Groups, preprint.

[19] Y. O. Hamidoune and J. O. Rødseth, An inverse theorem mod p, Acta Arith., 92 (2000), no. 3, 251-262.

[20] Y. O. Hamidoune, Subsets with a small sum II: The critical pair problem, European J. Combin. 21 (2000), no. 2, 231-239.

[21] Y. O. Hamidoune, Subsets with small sums in abelian groups I: The Vosper property, European J. Combin. 18 (1997), no. $5,541-556$.

[22] Y. O. Hamidoune, A. S. Lladó, and O. Serra, Vosperian and superconnected abelian Cayley digraphs, Graphs Combin., 7 (1991), no. 2, 143-152.

[23] Y. O. Hamidoune, Hyper-atoms and the critical pair Theory, preprint.

[24] Y. O. Hamidoune, Hyper-atoms and the Kemperman's critical pair Theory, preprint.

[25] V. Jungić, J. Nešetřil and R. Radoičić, Rainbow Ramsey Theory, Integers 5 (2005), no. 2, A9 (electronic).

[26] G. Károlyi, An inverse theorem for the restricted set addition in abelian groups, J. Algebra, 290 (2005), no. 2, 557-593.

[27] G. Károlyi, The Erdös-Heilbronn problem in abelian groups, Israel J. Math., 139 (2004), 349-359.

[28] J. H. B. Kemperman, On small sumsets in an abelian group, Acta Math., 103 (1960), 63-88.

[29] M. Kneser, Summenmengen in lokalkompakten abelschen gruppen, Math. Z., 66 (1956), 88-110.

[30] M. Kneser, Ein satz über abelsche gruppen mit anwendungen auf die geometrie der zahlen, Math. Z., 64 (1955), $429-434$.

[31] M. Kneser, Abschätzung der asymptotischen dichte von summenmengen, Math. Z., 58 (1953), 459-484.

[32] V. Lev, On small sumsets in abelian groups, Structure theory of set addition. Asterisque, No. 258 (1999), xv, 317321.

[33] V. Lev, Critical pairs in abelian groups and Kemperman's structure theorem, Int. J. Number Theory, 2 (2006), no. 3, 379-396.

[34] M. B. Nathanson, Additive Number Theory: Inverse Problems and the Geometry of Sumsets, Graduate Texts in Mathematics, 165, Springer-Verlag, New York, 1996.

[35] J. M. Pollard, A Generalisation of the Theorem of Cauchy and Davenport, J. London Math. Soc., (2) 8 (1974), 460-462.

[36] O. Serra and G. Zémor, On a generalization of a theorem by Vosper, Integers (2000), electronic.

[37] O. Serra, An isoperimetric method for the small sumset problem, Surveys in combinatorics 2005, London Math. Soc. Lecture Note Ser., 327, Cambridge Univ. Press, Cambridge (2005), 119-152.

[38] T. Tao and V. Vu, Additive combinatorics, Cambridge Studies in Advanced Mathematics 105, Cambridge University Press, Cambridge, 2006.

[39] A. G. Vosper, The critical pairs of subsets of a group of prime order, J. London Math. Soc., 31 (1956), 200-205.

[40] A. G. Vosper, Addendum to "The critical pairs of subsets of a group of prime order," J. London Math. Soc., 31 (1956), $280-282$.

Institut für Mathematik und Wissenschaftliches Rechnen, Karl-Franzens-Universität Graz, Heinrichstrasse 36, 8010 Graz, Austria

E-mail address: diambri@hotmail.com 